Aus der Poliklinik für Kieferorthopädie

(Prof. Dr. med. dent. P. Meyer-Marcotty)

im Zentrum für Zahn-, Mund- und Kieferheilkunde

der Medizinischen Fakultät der Universität Göttingen

\title{
Dreidimensionale Analyse der Kiefergelenkposition bei orthognather Chirurgie
}

Existiert ein Unterschied zwischen präoperativer und intraoperativer Kondylenposition?

\author{
INAUGURAL-DISSERTATION \\ zur Erlangung des Doktorgrades \\ für Zahnmedizin \\ der Medizinischen Fakultät der \\ Georg-August-Universität zu Göttingen
}

vorgelegt von

Johanna Katharina Trautmann

aus

Bremen

Göttingen 2021 
Dekan:

\section{Betreuungsausschuss}

Betreuer/in:

Ko-Betreuer/in:

\section{Prüfungskommission}

Referent/in:

Ko-Referent/in:

Drittreferent/in:

Datum der mündlichen Prüfung:
Prof. Dr. med. W. Brück

Prof. Dr. med. dent. P. Meyer-Marcotty

Prof. Dr. med. Dr. med. dent. H. Schliephake
Prof. Dr. med. dent. P. Meyer-Marcotty

PD Dr. med. Dr. med. dent. P. Kauffmann

Prof. Dr. mult. T. Meyer 
Hiermit erkläre ich, die Dissertation mit dem Titel "Dreidimensionale Analyse der Kiefergelenkposition bei orthognather Chirurgie - Existiert ein Unterschied zwischen präoperativer und intraoperativer Kondylenposition? " eigenständig angefertigt und keine anderen als die von mir angegebenen Quellen und Hilfsmittel verwendet zu haben.

Göttingen, den

(Unterschrift) 
Die Daten, auf denen die vorliegende Arbeit basiert, wurden teilweise publiziert:

Quast A, Santander P, Trautmann J, Moser N, Schliephake H, Meyer-Marcotty $\mathbf{P}$ (2020): A new approach in three dimensions to define pre- and intraoperative condylefossa relationships in orthognathic surgery - is there an effect of general anaesthesia on condylar position? Int J Clin oral Maxillofac Surg 49, 1303-1310

Erwin-Reichenbach-Förderpreis der Zahnärztekammer Sachsen-Anhalt 2019 Quast A, Santander P, Trautmann J: Evaluation prä- und intraoperativer Kondylenposition bei Dysgnathie-Patienten - eine prospektive klinische 3D Studie 


\section{Inhaltsverzeichnis}

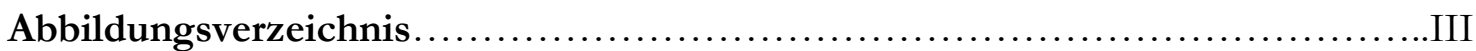

Tabellenverzeichnis .....................................................

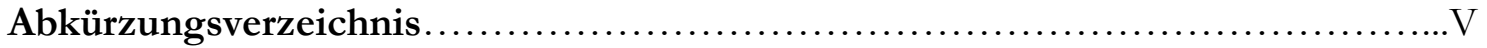

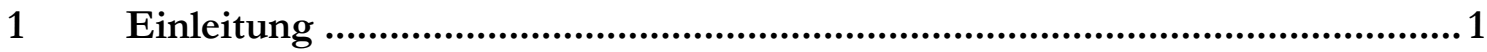

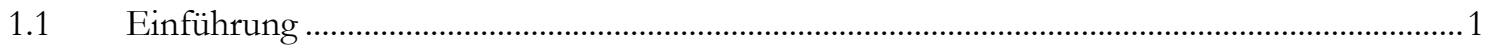

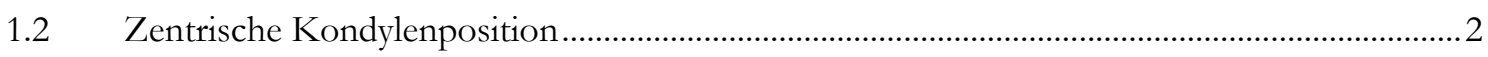

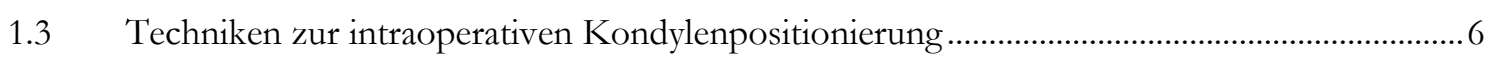

1.4 Interdisziplinäre Dysgnathietherapie der Universitätsmedizin Göttingen ...................................7

1.4.1 Kieferorthopädische Vorbehandlung..................................................................................

1.4.2 Operationsplanung, Durchführung und postoperative Behandlung ........................................11

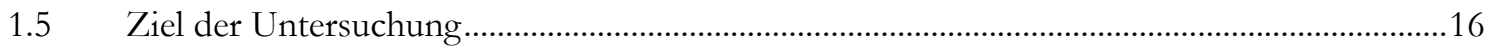

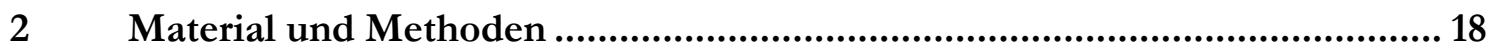

2.1 Patientenkollektiv und Rekrutierung ..................................................................................18

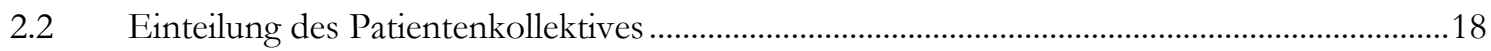

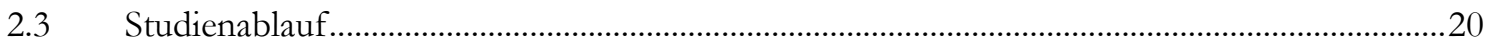

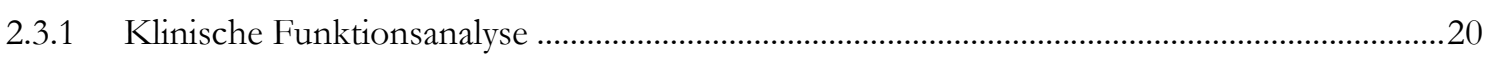

2.3.2 Bissnahme - Registrierung der zentrischen Kondylenposition ................................................20

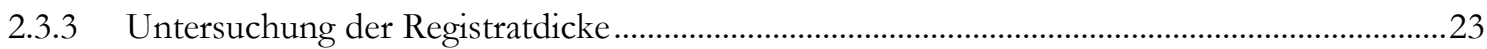

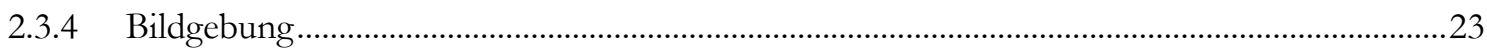

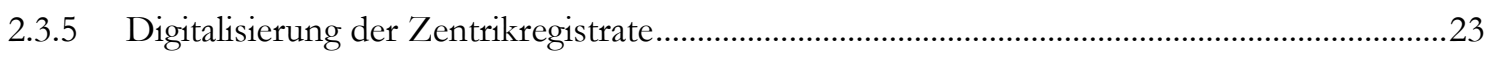

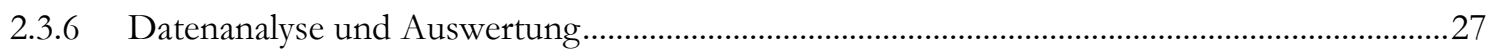

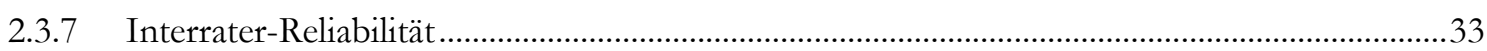

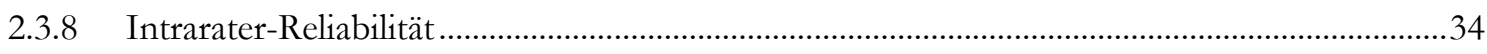

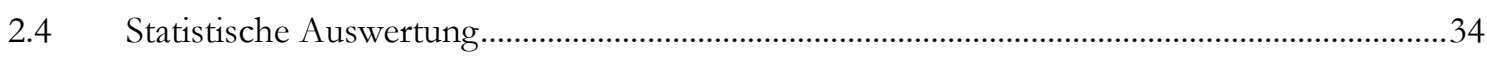

2.4.1 Statistische Auswertung der cephalometrischen Messungen .......................................................34

2.4.2 Statistische Auswertung der volumetrischen Übereinstimmung ..............................................34

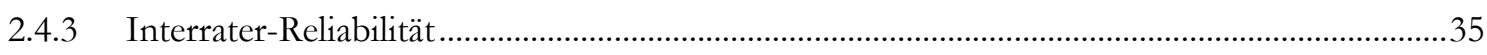

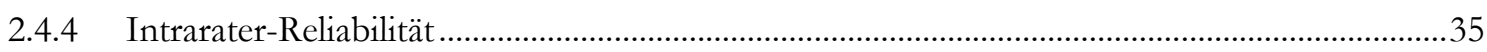

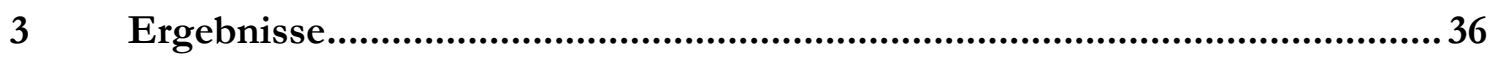

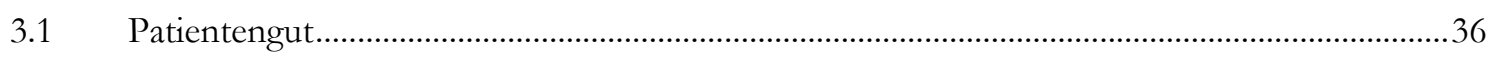

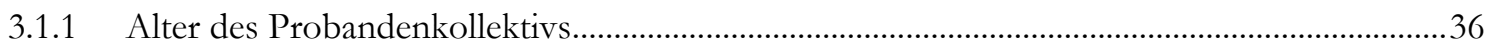

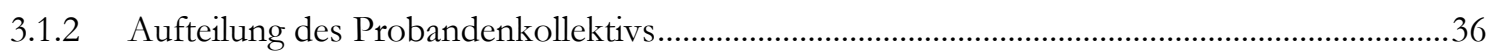

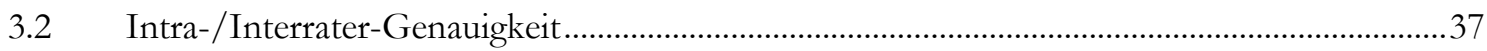

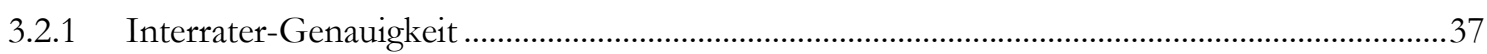

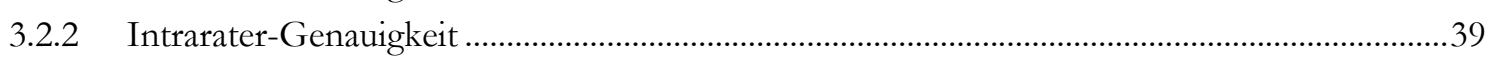

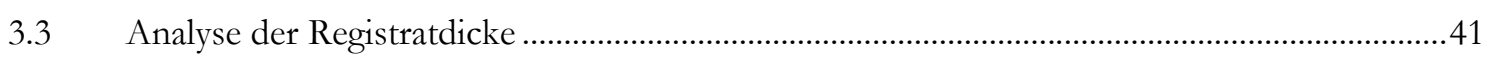




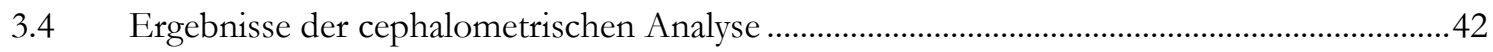

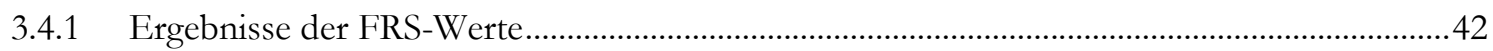

3.4.2 Ergebnisse der transversalen und dentalen cephalometrischen Messungen............................42

3.4.3 Ergebnisse der cephalometrischen Gelenkspaltmessungen ..................................................43

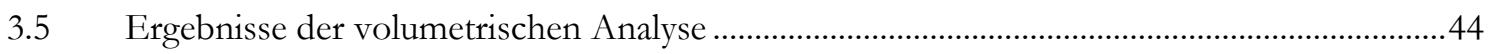

3.5.1 Volumetrische Ergebnisse des gesamten Probandenkollektivs ..............................................44

3.5.2 Ergebnisse der volumetrischen Subgruppenanalyse ................................................................46

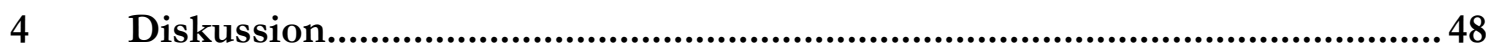

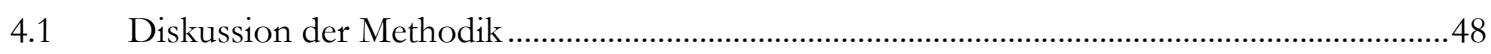

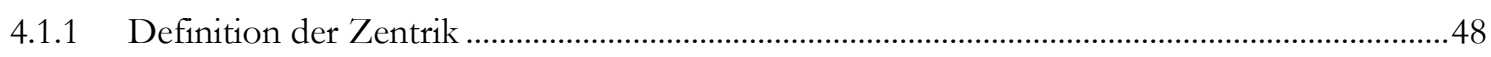

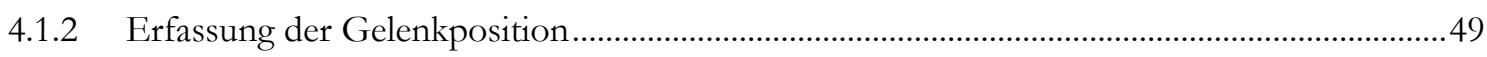

4.1.3 Problematik des Datensatzes und der Rekonstruktion ...............................................................50

4.1.4 Fehlerquellen und Problematik der cephalometrischen Analyse...........................................50

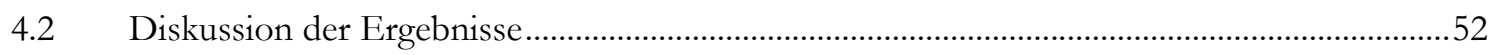

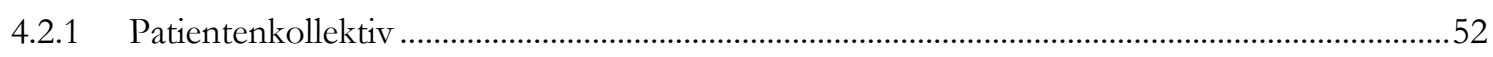

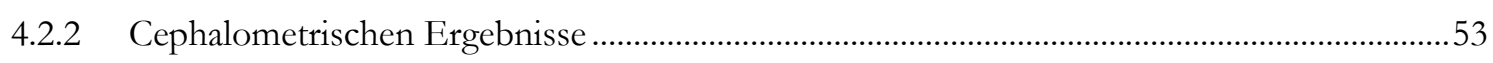

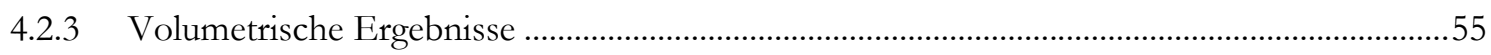

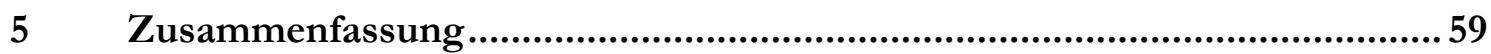

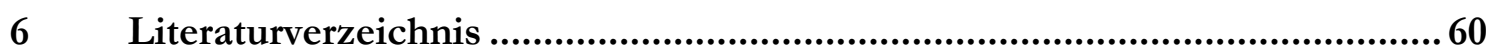




\section{Abbildungsverzeichnis}

Abbildung 1: Röntgenologische Darstellung der zentrischen Kondylenposition.................................2

Abbildung 2: Einseitig verschobene Kondylenposition einer stark laterognathen Patientin............... 4

Abbildung 3: Einseitig verschobene Kondylenposition durch okklusale Interferenzen (lateraler

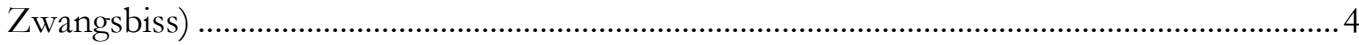

Abbildung 4: Splintdmethodik nach Luhr und Schwestka ............................................................

Abbildung 5: Fernröntgenseitenbild und cephalometrische Analyse des Patientenfalles.....................8

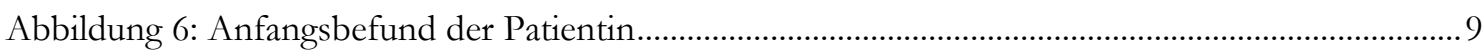

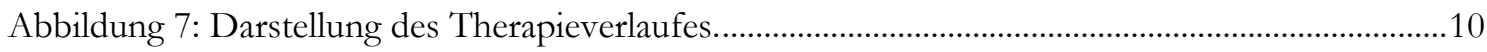

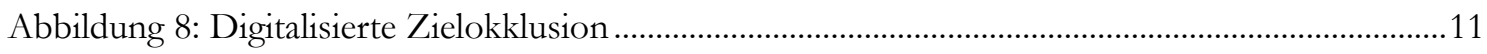

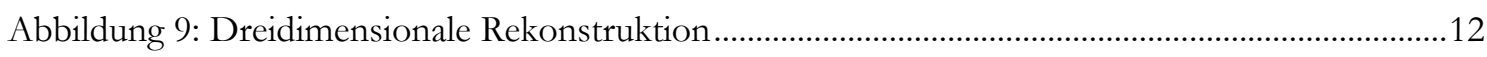

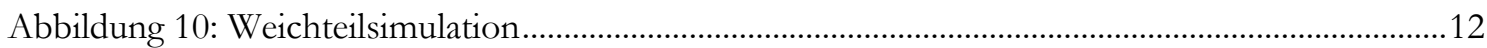

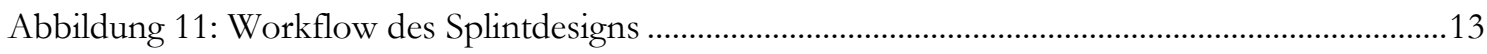

Abbildung 12: Darstellung der Fixierung des gelenktragenden Anteils............................................13

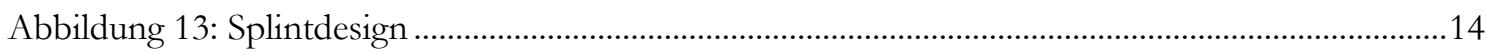

Abbildung 14: Intraorale Passung eines Zentriksplintes ...............................................................15

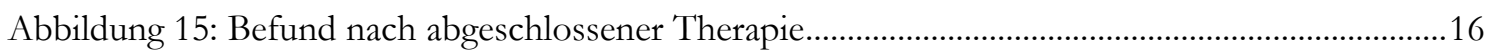

Abbildung 16: Herstellung und Bissnahme des Zentrikregistrates mit Primobyte .............................21

Abbildung 17: Lagerung des Patienten für die Zentriknahme .........................................................21

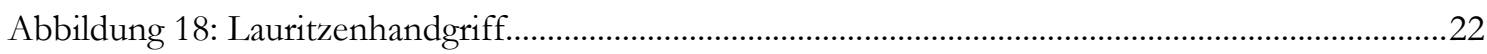

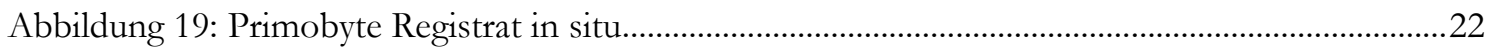

Abbildung 20: Rekonstruktion der Schädelmodelle......................................................................23

Abbildung 21: Rekonstruktion der Kondylen...................................................................................24

Abbildung 22: Modelle mit Primobyte-Registrat eines Patienten mit ausgeprägter skelettaler

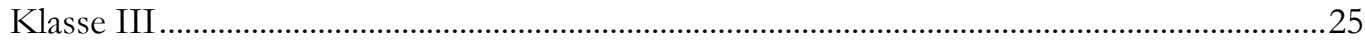

Abbildung 23: Herstellung des Composite Modelles Maxilla/Cranium................................................25

Abbildung 24: Einstellung der Okklusion mithilfe der gescannten präoperativen

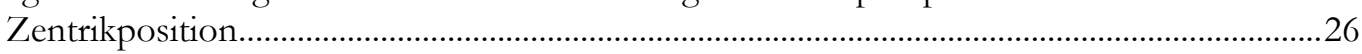

Abbildung 25: Herstellung der Unterkiefer Composite Modelle.......................................................26

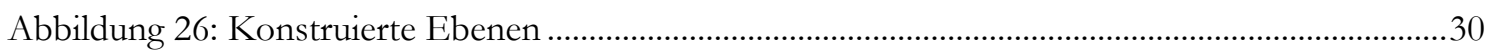

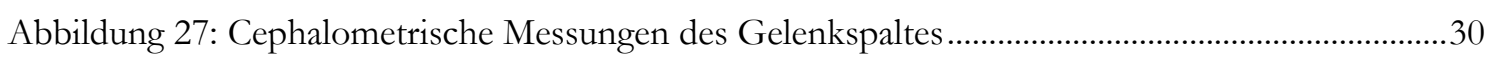

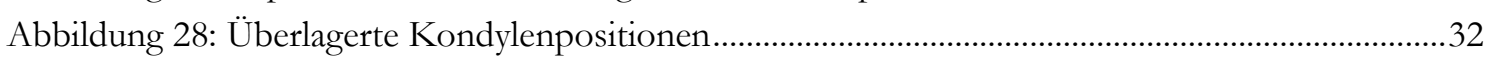

Abbildung 29: Darstellung der deckungsgleichen Bereiche der prä-und intraoperativen

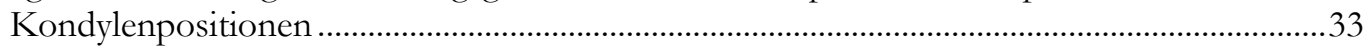

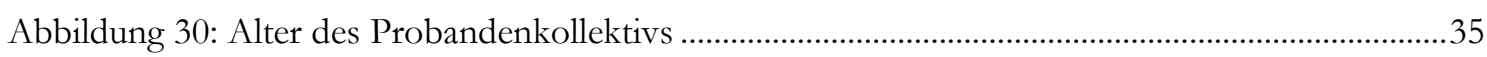

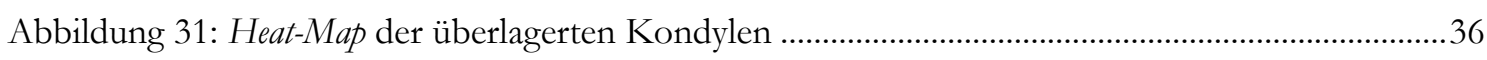

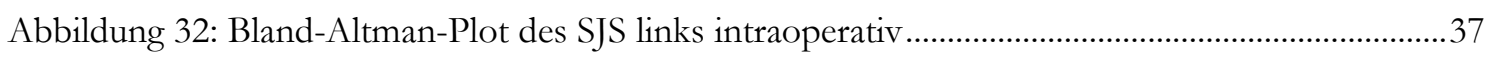

Abbildung 33: Bland-Altman-Plot des AJS rechts intraoperativ .......................................................38

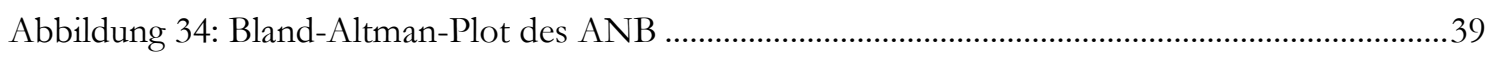

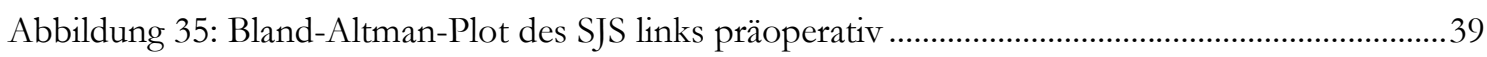

Abbildung 36: Darstellung der dentalen Abweichungen ..................................................................41

Abbildung 37: Kollidierendes Volumen der prä-und intraoperativen Gelenkposition......................43

Abbildung 38: Visuelle Bewegungsrichtung der Kondylen ..............................................................44

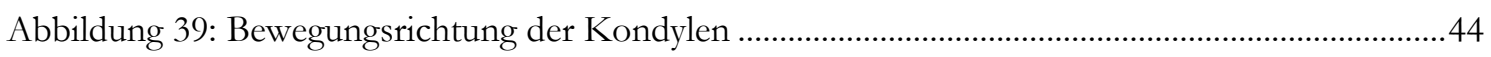

Abbildung 40: Darstellung der Gelenkposition der Subgruppen ....................................................45 


\section{Tabellenverzeichnis}

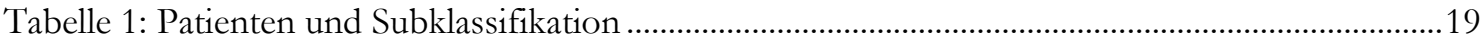

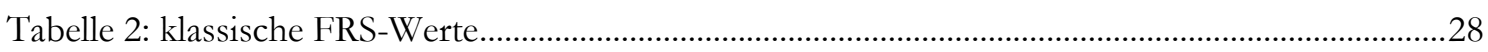

Tabelle 3: cephalometrische Orientierungspunkte für die Vermessung der Kondylenposition prä- und intraoperativ ....................................................................................................28

Tabelle 4: cephalometrische Orientierungspunkte zur Vermessung der dentalen Verhältnisse

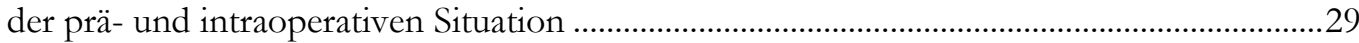

Tabelle 5: konstruierte Ebenen zur cephalometrischen Analyse .........................................................30

Tabelle 6: Cephalometrische Messungen des Gelenkspaltes prä- und intraoperativ und der dentalen Referenzpunkte.

Tabelle 7: Ergebnisse der Abweichungen der kondylären Rekonstruktion zwischen

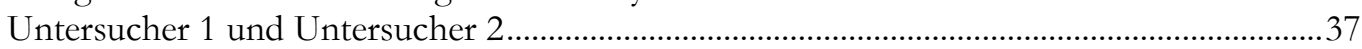

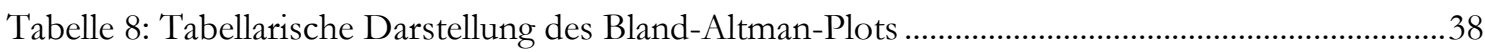

Tabelle 9: Tabellarische Darstellung des Bland-Altman...........................................................................39

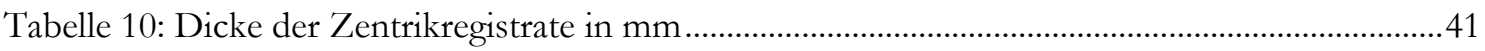

Tabelle 11: cephalometrische Ergebnisse der FRS Werte.......................................................................42

Tabelle 12: Ergebnisse der transversalen und dentalen cephalometrischen Messungen ....................42

Tabelle 13: Ergebnisse der linearen Gelenkspaltmessung ................................................................43

Tabelle 14: Ergebnisse des volumetrischen Vergleiches der Kondylen................................................44

Tabelle 15: Ergebnisse der volumetrischen Subgruppenanalyse Symmetrie......................................46

Tabelle 16: Ergebnisse der volumetrischen Subgruppenanalyse der skelettalen Klasse .....................45 


\section{Abkürzungsverzeichnis}

ASA Amercian society of Anesthesiologists, hier ASA-Klassifikation

CMD Craniomandibuläre Dysfunktion

DICOM digital imaging and communications in medicine

DGFDT Deutsche Gesellschaft für Funktionsdiagnostik und -therapie

DGZMK Deutsche Gesellschaft für Zahn-, Mund- und Kieferheilkunde

DVT Digitale Volumentomographie

$\mathrm{HU}$ houndsfield unit

$\mathrm{M}$ Mittelwert

MRT Magnetresonanztomographie

n Anzahl

ROI region of interest

SD Standardabweichung

STL standart triangulation language (Dateiformat zur Beschreibung der Oberflächen von 3D Körpern)

UMG Universitätsmedizin Göttingen

ZMK Zahn-, Mund-, Kieferheilkunde 


\section{$1 \quad$ Einleitung}

\section{$1.1 \quad$ Einführung}

Im Gegensatz zur Eugnathie, besteht bei einer Dysgnathie eine ausgeprägte Fehlstellung der Zähne und Kiefer zueinander. Hier unterscheiden sich dentoalveoläre Anomalien, die meist durch eine rein kieferorthopädische Behandlung korrigiert werden können, von skelettalen Anomalien, deren Korrektur häufig eine Kombination aus kieferorthopädischer Therapie und einer ergänzenden Dysgnathiechirurgie, mit der operativen Verlagerung der Kiefer zueinander, erfordert. Eine Indikation zu einer solchen kieferorthopädisch-kieferchirurgischen Therapie ist immer dann gegeben, wenn die Ausprägung der skelettalen und dentoalveolären Anomalie über die Behandlungsmöglichkeiten einer rein kieferorthopädischen Therapie hinausgeht. Wenn weder Wachstum ausreichend genutzt werden kann, noch eine Camouflagetherapie zu einem zufriedenstellenden Ergebnis führt, stößt die rein kieferorthopädische Behandlung an ihre Grenzen. Welche Behandlungsstrategie für den Patienten indiziert ist, muss der Kieferorthopädie nach Analyse der sagittalen, vertikalen und transversalen skelettalen und dentoalveolären Verhältnisse entscheiden. Eine klar definierte Grenze, ab wann eine Kombinationstherapie notwendig ist, gibt es nicht. Einige Autoren haben Schemata entwickelt (Proffit et al. 2019; Segner und Hasund 1998) um eine Therapieplanung zu erleichtern, jedoch ist jede Entscheidung höchst individuell und muss sowohl von Behandler als auch Patient getragen werden.

Seit Begründung der Dysgnathiechirurgie Ende des 19. Jahrhunderts in den Vereinigten Staaten durch Angle (1898), Whipple (1898) und Blair (1906) und ihrer „St. Louis Operation" zur Korrektur einer Prognathie, haben sich unzählige Untersuchungen mit deren Indikationen, Operationsmethodiken, Osteosynthesevarianten und der prächirurgischen kieferorthopädischen Dekompensation befasst (Steinhäuser 1996). Zwar müsste man korrekterweise in diesem Zuge auch Hullihen (1849) erwähnen, der mit einer alveolären Osteotomie der Mandibula bereits 1849 einen erworbenen skelettal offenen Biss korrigierte, jedoch wird dieser in der Literatur meist nicht als „Urvater“ der orthognathen Chirurgie genannt.

Nachdem Whipple (1898) im 19. Jahrhundert noch mit zahlreichen Komplikationen zu kämpfen hatte, dauerte es knapp ein Jahrhundert bis die orthognathe Chirurgie ab den 1980er-Jahren zur Standardtherapie für skelettale Anomalien der Kiefer und des Gesichtsschädels geworden ist. Diese Entwicklung ist letztendlich sicherlich den technischen Fortschritten durch Obwegeser (1964), der Modifikation nach Dal Pont (1961), Hunsuck (1968) sowie Bell und Epker (1976) in den 60er und 70er Jahren zu verdanken. Zusätzlich 
haben auch die Verbesserungen der Anästhesiemöglichkeiten, der begleitenden medikamentösen Therapie, der funktionsstabilen Osteosynthese und natürlich der kieferorthopädischen Behandlung zur Implementierung in den klinischen Alltag geführt (Proffit et al. 1992).

Ein zentrales Thema in der Dysgnathiechirurgie stellt die Sicherung einer physiologischen Positionierung der Kiefergelenke in der Fossa articularis dar. Um ein vorhersehbares und stabiles Ergebnis zu erzielen, steht häufig die zentrische Kondylenposition, deren präoperative Registrierung und intraoperative Sicherung zur Debatte. Mit diesem besonders kontrovers diskutierten Thema beschäftigt sich auch die vorliegende Arbeit.

\subsection{Zentrische Kondylenposition}

Nach Definition der Deutschen Gesellschaft für Funktionsdiagnostik- und therapie (DGFDT) aus dem Jahre 2005 ist die zentrische Kondylenposition die „kranio-ventrale, nicht seitenverschobene Position beider Kondylen bei physiologischer Kondylus-DiskusRelation und physiologischer Belastung der beteiligten Gewebestrukturen“ (DGZMK 2005) (Abbildung 1). Diese Kondylenposition ist fachübergreifend für Zahnärzte, Kieferorthopäden und Mund-, Kiefer- und Gesichtschirurgen relevant. Eine pathologische Kondylenposition kann auch bei Patienten ohne ausgeprägte Dysgnathien Beschwerden verursachen, wie z. B. Bewegungseinschränkungen und Schmerzen im Bereich des Gelenkes bei Bewegung und in Ruhe, Druckdolenzen oder entzündlich-degenerative Prozesse (Paknahad und Shahidi 2015; Weinberg 1983) (Abbildung 2, Abbildung 3). Im Rahmen einer CMD-Therapie (Craniomandibuläre Dysfunktion) werden diese komplexen Symptome häufig mit einer okklusalen Schienentherapie und ergänzender Physiotherapie behandelt.

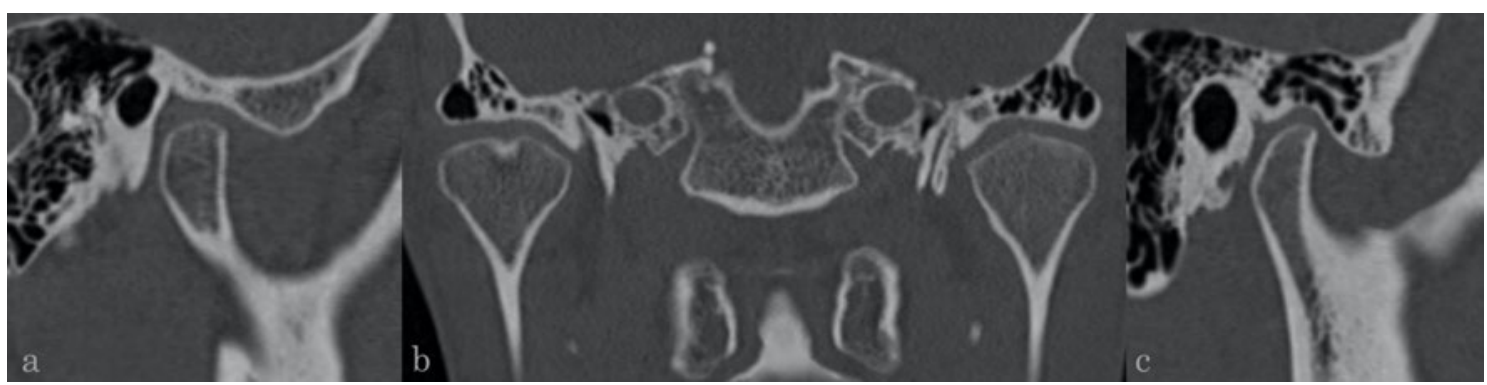

Abbildung 1: Röntgenologische Darstellung der zentrischen Kondylenposition a) Kondylus rechts in der Sagittalebene, b) Kondylen in der Koronalebene und c) Kondylus links in der Sagittalebene.

Auch im Rahmen der Dysgnathiechirurgie nimmt die Kondylenpositionierung eine wichtige Rolle ein. Im Vergleich zu regulären zahnmedizinischen Eingriffen ist sie durch das OPProzedere an sich erschwert. Während der Osteotomie des Unterkiefers, wird dieser in zwei 
gelenktragende und einen zahntragenden Anteil gespalten. Der zahntragende Anteil lässt sich über einen Operationssplint in die Zielposition führen, währenddessen sind die proximalen, gelenktragenden Anteile frei beweglich. Durch die Narkose kommt es zudem zu einem Erschlaffen der Kaumuskulatur und damit zu einem Haltverlust im Gelenk. Diese Faktoren können unbemerkt zu einer Fehlpositionierung der Gelenke führen und beeinträchtigen die rein manuelle Positionierung der Kondylen (Schwestka-Polly 2004).

Laut Luhr et al. (1986), Worms et al. (1980) und Van Sickels et al. (2000) ist die physiologische Beziehung zwischen Kondylus und Fossa jedoch ein entscheidender Faktor für die postoperative Langezeitstabilität des Operationsergebnisses. Die Langzeitstabilität einer kombiniert kieferorthopädisch-kieferchirurgischen Therapie ist eines der essentiellsten Kriterien für eine erfolgreiche Behandlung (Luhr et al. 1986; Paulus 1983). In diesem Zusammenhang zählt laut Epker und Wessberg (1982) das skelettale Rezidiv zur häufigsten Begleiterscheinung einer Unterkiefervorverlagerung. Bettega et al. (2002) bestätigen einen Zusammenhang zwischen der Qualität der sagittalen Reposition der Kiefergelenke und der knöchernen Stabilität. Neben der sagittalen Position seien die funktionellen Ergebnisse zudem abhängig vom Torque des Kondylus.

Eggensperger et al. (2006), Blomqvist et al. (1997) und Ellis und Hinton (1991) zeigten in ihren Untersuchungen eine Korrelation zwischen der intraoperativen Fehlpositionierung des Gelenkes und postoperativen Komplikationen. So führt eine Distraktion des Kiefergelenkspaltes und eine anteriore Positionierung des Kondylus zu einem frühzeitigen Rezidiv, während eine retrale Positionierung eher kondyläre Resorptionen und ein Spätrezidiv zur Folge hat. Eine Verringerung des posterioren Kiefergelenkspaltes steigert zudem die Kräfte auf das Gelenksystem (Yang et al. 2013) was die Wahrscheinlichkeit zur Entwicklung von Craniomandibulären Dysfunktionen erhöhen kann (Pullinger et al. 1986; Saka et al. 2004; Shokri et al. 2019). Laut Jędrzejewski et al. (2015) gehört die CMD zu den zweithäufigsten postoperativen Komplikationen und betrifft $13,54 \%$ der Dysgnathiepatienten. Postoperativ kann es sowohl zu artikulären Geräuschen wie Knacken oder Reiben als auch zu Schmerzen im Gelenkbereich kommen (Hori et al. 1999; Wolford et al. 2003).

Auch strukturell kommt es postoperativ zu Anpassungsprozessen im Bereich des Kiefergelenks. Wie schon von Häupl (1960) und Eschler (1963) beschrieben, ist das stomatognathe System ein Zusammenspiel von verschiedenen Geweben die über ihre Funktion miteinander verbunden sind und auf funktionelle Reize physiologischer und pathologische Art reagieren. Während Brooks et al. (1992) bei asymptomatischen Patienten nur minimale adaptive Veränderungen der kondylären Oberfläche gefunden hat, fanden Yamada et al. (2001) bei Patienten, die sich einer orthognathen Chirurgie unterzogen, eine deutlich höhere Rate an Umbauprozessen. Diese Veränderungen waren zusätzlich progressiver Art, wie z. B. Erosion und die Bildung von Osteophyten. Eine 
computertomographische Untersuchung von Claus et al. (2019) über den Einfluss von bimaxillären Umstellungsosteotomien auf Veränderungen der kondylären Oberfläche zeigte aber keine signifikanten Zusammenhänge. Jedoch beobachteten die Autoren, dass im posterioren und superioren Bereichen des Kondylus stärkere Resorptionen auftraten.

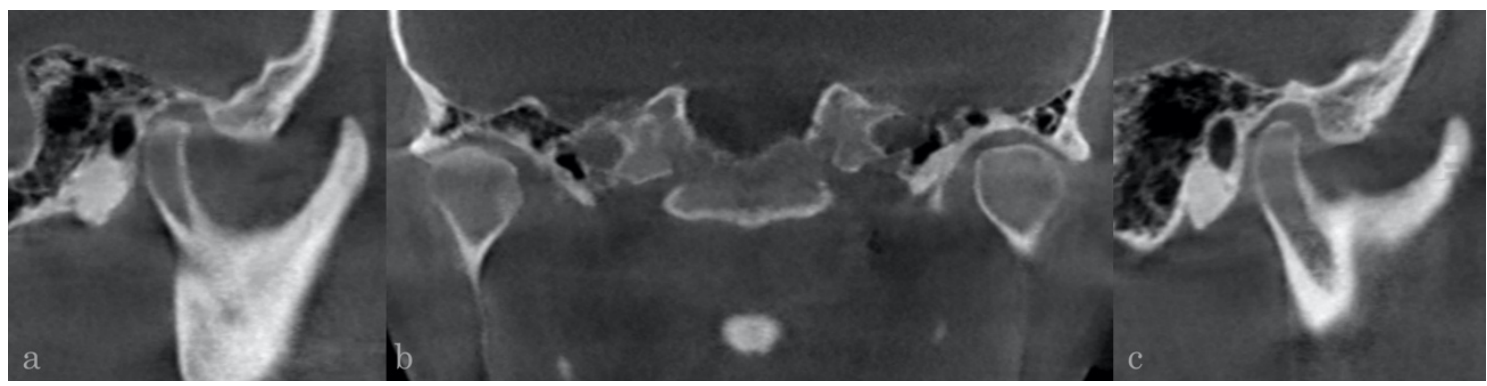

Abbildung 2: Einseitig verschobene Kondylenposition einer stark laterognathen Patientin a) stark retrale und caudale Position des rechten Kondylus in der Sagittalebene b) Kondylen in der Koronalebene und c) linker Kondylus in zentrischer Position in der Sagittalebene.

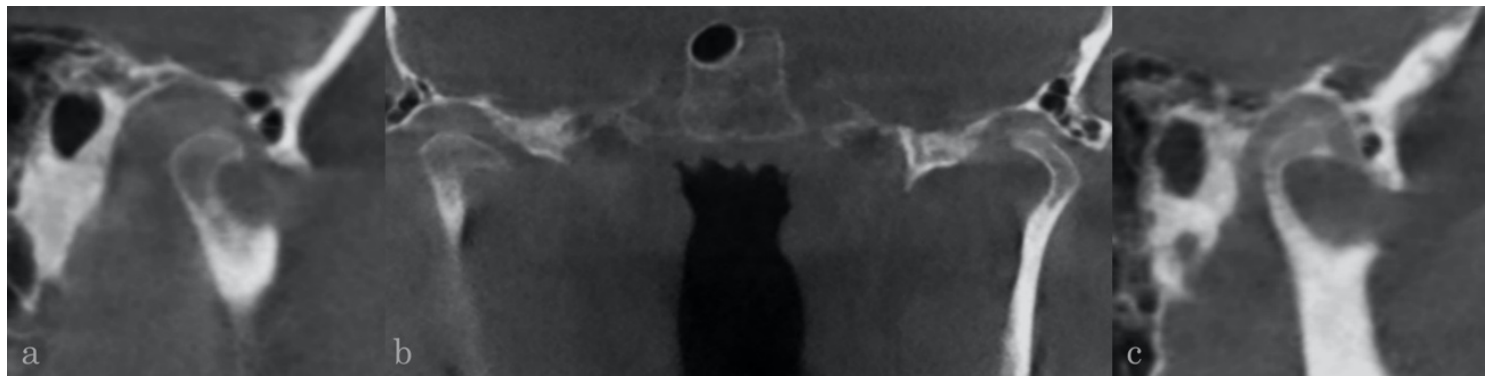

Abbildung 3: Einseitig verschobene Kondylenposition durch okklusale Interferenzen (lateraler Zwangsbiss) a) rechter Kondylus in anteriorer Position in der Sagittalebene b) beide Kondylen in der Koronalebene mit deutlichen Formunterschieden c) linker Kondylus in der Sagittalebene.

$\mathrm{Ob}$ eine kombiniert kieferorthopädisch-kieferchirurgische Therapie einen positiven oder negativen Effekt auf CMD-Symptomatiken hat, wird nach wie vor diskutiert. In der systematischen Literaturübersicht von Al-Moraissi et al. (2017) zeigt sich, dass eine kieferorthopädisch-kieferchirurgische Therapie bei einem Großteil der Patienten mit CMDSymptomatiken zu einer Linderung der Symptome beitragen kann. Allerdings ist auch bei einem geringen präoperativ asymptomatischen Anteil der Patienten postoperativ eine Verschlechterung bzgl. der Kiefergelenkfunktion eingetreten (Aoyama et al. 2005; Wolford et al. 2003). Zu beachten geben Al-Moraissi et al. (2017) jedoch, dass in den untersuchten Studien weder Hinweise auf die postoperative kieferorthopädische Behandlung noch auf die Verwendung von Splinten oder Physiotherapie gegeben werden.

Es ist dennoch davon auszugehen, dass der korrekten Registrierung und Positionierung der Kiefergelenke während der Dysgnathiechirurgie eine entscheidende Bedeutung am Therapieerfolg zu kommt. Die Registrierung der zentrischen Kondylenposition kann klinisch 
zu Herausforderungen führen. Eine Vielzahl an Studien, unabhängig von vorliegenden Dysgnathien, haben bis dato verschiedenste Registriertechniken untersucht, allerdings ist laut Utz et al. (2002) keine Technik klinisch überlegen. Etz et al. (2012) weisen indes auf eine essentielle Reproduzierbarkeit der zentrischen Kondylenposition als wesentliche Voraussetzung bei der Auswahl der Verfahrenstechniken hin. Diese stellt jedoch bei der Registrierung ein erhebliches Problem dar. Zusätzlich findet sich zwischen der, durch die Interkuspidation determinierte Kondylenposition und der Zentrik eine signifikante Diskrepanz (Böhm et al. 1995; Keshvad und Winstanley 2001; Weffort et al. 2010).

Darüber hinaus stellt auch die Kaumuskulatur einen deutlichen Einflussfaktor auf die Position der Kiefergelenke dar. Laut Wang et al. (2009) und Jimenez (1989) zeigt sich, als physiologische Auswirkung einer Änderung der Mandibulaposition auch eine angepasste Muskelaktivität. Schindler et al. (2009) fanden heraus, dass selbst geringe vertikale Veränderungen eine detektierbare Differenz in der Muskelaktivität und der auf das Gelenksystem wirkenden Kräfte verursachen können. Diese Studien wurden an Probandenkollektiven durchgeführt, bei denen kein Anhalt auf skelettale Anomalien vorliegt. Patienten mit ausgeprägten Kieferfehlstellungen zeigen jedoch vermehrt atypische Muskelund Gelenkfunktionen und neigen zu CMD (Moss und Chalmers 1974; Toll et al. 2010). Besonders Patienten mit einer skelettalen Klasse II Anomalie weisen häufig eine Kiefergelenkkompression mit einhergehender Degeneration der Kondylen auf (Toll et al. 2010). Auch Solberg et al. (1986) konnte den Zusammenhang zwischen Anomalien der Okklusion und Veränderung des Gelenkapparates belegen. Eine erhöhte Inzidenz von Kiefergelenkdysfunktionen wird unter anderem mit der Malokklusion einer skelettalen Klasse II/2 diskutiert (Schupp et al. 1992).

Zudem führen laut Zak et al. (1992) und Yaghmaei et al. (2013) unterschiedliche Muskeltoni zu signifikanten Unterschieden in der Kondylenposition. Beide Studien untersuchten den Einfluss von Muskelrelaxantien im Rahmen einer Allgemeinanästhesie auf die zentrische, okklusale Position. Da laut Studienlage Patienten mit skelettalen Bissanomalien vermehrt atypische Muskelfunktionen aufweisen, ist davon auszugehen, dass eine Allgemeinnarkose, wie sie bei Umstellungsosteotomien zum Einsatz kommt, besonders bei diesen Patienten signifikanten Einfluss hat (Bamber et al. 1999). Zak et al. (1992) bestätigen diese Annahme und erklären den Einsatz von Barbituraten ohne Muskelrelaxantien bereits für ausreichend um eine Positionsänderung hervorzurufen. Yaghmaei et al. (2013) fanden nur bei Klasse II Patienten eine signifikante Auswirkung der Allgemeinanästhesie. Dagegen gibt es laut Borba et al. (2014) keine relevanten Veränderungen zwischen der zentrischen Kondylenposition und der intraoperativen Position. 


\subsection{Techniken zur intraoperativen Kondylenpositionierung}

Eine Möglichkeit die Abweichungen der Kondylenposition prä- und intraoperativ zu vermeiden, stellt die intraoperative Kondylenpositionierung dar. Sie dient der Sicherung der präoperativen Kondylenposition vor, während und über den chirurgischen Eingriff hinaus und soll so skelettale Rezidive und symptomatische CMD vermeiden. Bereits Leonard (1976) stellte eine Technik zur intraoperativen Positionierung vor. Es folgte in den darauffolgenden Jahrzenten eine Vielzahl von Möglichkeiten. Während die meisten Techniken auf manuelle oder computerbasierte splintunterstütze, okklusale Registrierungen zurückgreifen (Cortese et al. 2019; Luhr 1989; Nova et al. 2017; Schwestka et al. 1990), gibt es in der Literatur auch speziellere Lösungen, wie z. B. die von Politi et al. (2007) beschriebene intraoperative Reduzierung der Anästhesietiefe, die computerunterstützte optische Lokalisierung (Bettega et al. 1996; Bettega et al. 2002) oder die Nutzung von intraoperativer Sonographie (Gateno et al. 1993). Alle Varianten zielen jedoch auf die intraoperative Sicherung des gelenktragenden Anteils des Unterkiefers ab.

Ein Review von Costa et al. (2008) untersuchte hierzu anhand von Studien zwischen 1990 und 2007 die Notwenigkeit von Kondylenpositionierungshilfen während einer Dysgnathiechirurgie im Zusammenhang mit skelettalen Rezidiven und postoperativer CMD. Laut Autoren besteht keine Evidenz zur Nutzung von Hilfsmitteln, jedoch kamen nur bei wenigen der untersuchten Studien Hilfsmittel zum Einsatz. Eine manuelle intraoperative Positionierung hat sich klinisch zwar etabliert, die Verwendung von individuell hergestellten Hilfen jedoch nicht, da die meisten Methodiken zum Gebrauch von splintunterstützten und okklusalen Registrierungen zeitintensiv sind und zahntechnische Vorarbeit benötigen.

Lindqvist und Söderholm (1988) publizierten eine einfache und zeitsparende Methodik zur Positionierung des gelenktragenden Anteils: als zentrische Kondylenposition wurde die präoperative, habituelle Okklusion angenommen, die mit einem Wachsbiss registriert wurde. Intraoperativ wurden die Kiefer mit Hilfe des Wachsbisses rigide fixiert und eine Osteosyntheseplatte zwischen Ramus und Maxilla bzw. dem Stützpfeiler des Os zygomaticum angebracht. Laut Autoren ist eine mukosale Präparation des Oberkiefers nicht notwendig, sodass die Schrauben direkt eingebracht werden können.

Auch Reyneke und Ferretti (2002) nutzen zur Positionierung des Kondylus eine zeitsparende Methodik unter Verwendung eines „condylar seating tool“. Nach der Durchtrennung des Unterkiefers wird das proximale Segment mit dem Instrument nach posterior und inferior gedrückt, während von extraoral mit leichtem digitalen Druck der Unterkieferwinkel nach anterior und superior bewegt wird.

Cortese et al. (2019) machen sich hingegen die Möglichkeit des computerassistierten Designs und Herstellung von chirurgischen Hilfsmitteln zu Nutze. Neben eines Splintes für die Zielokklusion, verwenden die Autoren zur Positionierung des proximalen Anteils der 
Mandibula zwei individuelle, digitale Positionierungshilfen die zwischen den letzten Molaren und den aufsteigenden Ästen angebracht werden. Sie umfassen die Kurvatur der Äste und die distalen Flächen der Molaren. Auch hier wird die Schablone fest mit dem Ramus verschraubt.

Trotz dieser Vielzahl an Techniken hat sich der Einsatz von Hilfsmitteln bis dato im klinischen Alltag noch nicht etabliert (Costa et al. 2008; Ellis 1994). Gründe hierfür liegen laut Ellis (1994) am erhöhten Zeitaufwand und dem Können des Chirurgen, die Kondylen eigenhändig zu positionieren.

An der Universitätsmedizin Göttingen (UMG) etablierten Luhr (1989) und Schwestka et al. (1990) eine Splintmethodik zur Übertragung der klinisch registrierten Kondylenposition in das operative Setting (Abbildung 4).
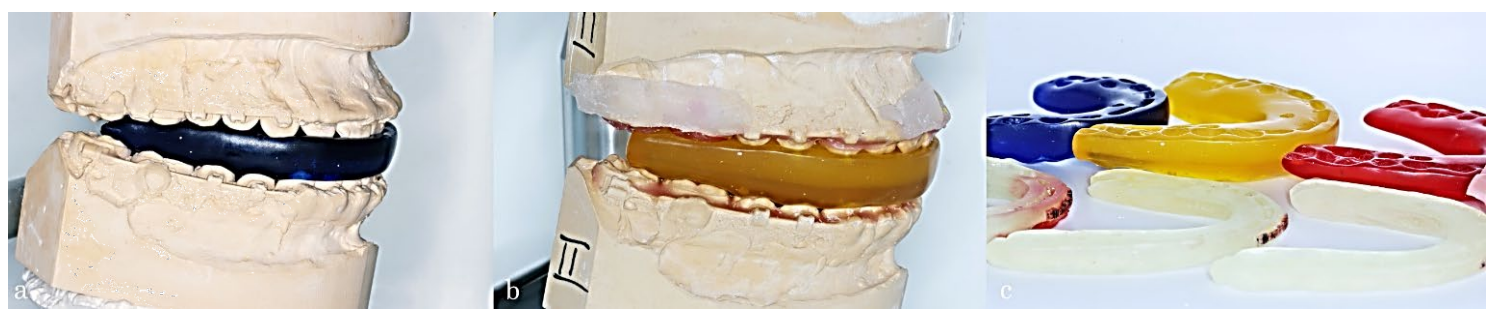

Abbildung 4: Splintmethodik nach Luhr und Schwestka a) blauer Zentriksplint b) Intermediate Splint c) Vergleich der konventionellen Splinten (bunt) und den aktuell genutzten Digitalen (weiß-transparent).

In den letzten Jahren konnte dieses Planungsprocedere auf ein modernes digitales Planungssystem übertragen werden. Das heutige digitale Splint-Design erfolgt anhand einer Modelloperation mithilfe einer medizinischen Software, an dem die einzelnen Schritte der Verlagerung simuliert werden. Dieses Konzept wird nachfolgend zusammen mit dem allgemeinen Operationsablauf detailliert dargestellt.

\subsection{Interdisziplinäre Dysgnathietherapie der Universitätsmedizin Göttingen}

\subsubsection{Kieferorthopädische Vorbehandlung}

Patienten mit ausgeprägter Kieferfehlstellung und einer Indikation zur Kombinationstherapie durchlaufen am Zentrum für Zahn-, Mund-, Kieferheilkunde der UMG einen standardisierten Ablauf zur Korrektur der Anomalien. Nach initialer Vorstellung in der interdisziplinären Dysgnathiesprechstunde und Entscheidung des Patienten für die Therapie erfolgt zunächst die kieferorthopädische Vorbehandlung. 
Das interdisziplinäre, ausführliche Aufklärungsgespräch ist unabdingbar. Da es sich um eine zeitintensive Therapie handelt, die neben den kieferorthopädischen auch besonders chirurgische Risiken- und Nebenwirkungen bringt, sollte dem Patienten ausreichend Informationen und Bedenkzeit zur Verfügung stehen.

Die kieferorthopädische Vorbehandlung hat die Auflösung der natürlichen dentoalveolären Kompensation der skelettalen Dysgnathie zur Aufgabe (Abbildung 5, Abbildung 6). Die Phase der Dekompensation verfolgt zwei Ziele: Zum einen dient sie der Anpassung der dentalen an die skelettale Situation und führt so zur Optimierung der operativen Verlagerungsstrecken, zum anderen schafft sie Spielraum zur dentalen Kompensation eines potentiellen postoperativen Rezidives (Proffit et al. 2019). Dabei führt die präoperative Dekompensation häufig zu einer Verschlechterung des extraoralen Erscheinungsbildes des Patienten (Troy et al. 2009) und einer initialen Reduktion der Kaufunktion durch Verstärkung der dentoalveoläre Dysgnathie.

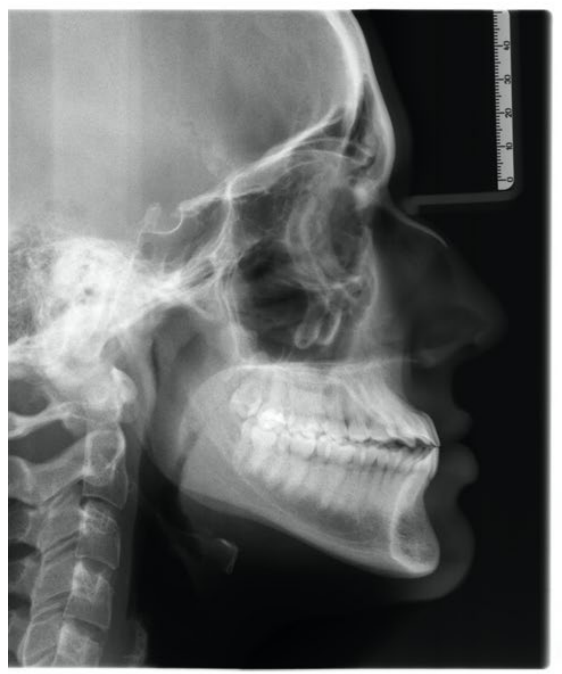

\begin{tabular}{lll}
\hline Variabel & & Interpretation \\
& & \\
SNA & $85,2^{\circ}$ & Maxilla prognath \\
SNB & $87,5^{\circ}$ & Mandibula prognath \\
ANB & $-2,2^{\circ}$ & Skelettale Klasse III \\
Wits & $-11,3 \mathrm{~mm}$ & Skelettale Klasse III \\
NL-NSL & $8,5^{\circ}$ & Keine Neigung der Maxilla \\
ML-NSL & $29,5^{\circ}$ & Keine Neigung der Mandibula \\
ML-NL & $21,0^{\circ}$ & Neutrale Relation \\
OK1-NA & $39,6^{\circ}$ & Stark protrudierte \\
UK1-NB & $37,0^{\circ}$ & Oberkieferinzisivi \\
& & \\
\hline
\end{tabular}

Abbildung 5: Fernröntgenseitenbild und cephalometrische Analyse des dargestellten Patientenfalles. Anhand der stark protrudierten Oberkieferfrontzähne zeigt sich die ausgeprägte dentale Kompensation der skelettalen Klasse III. 


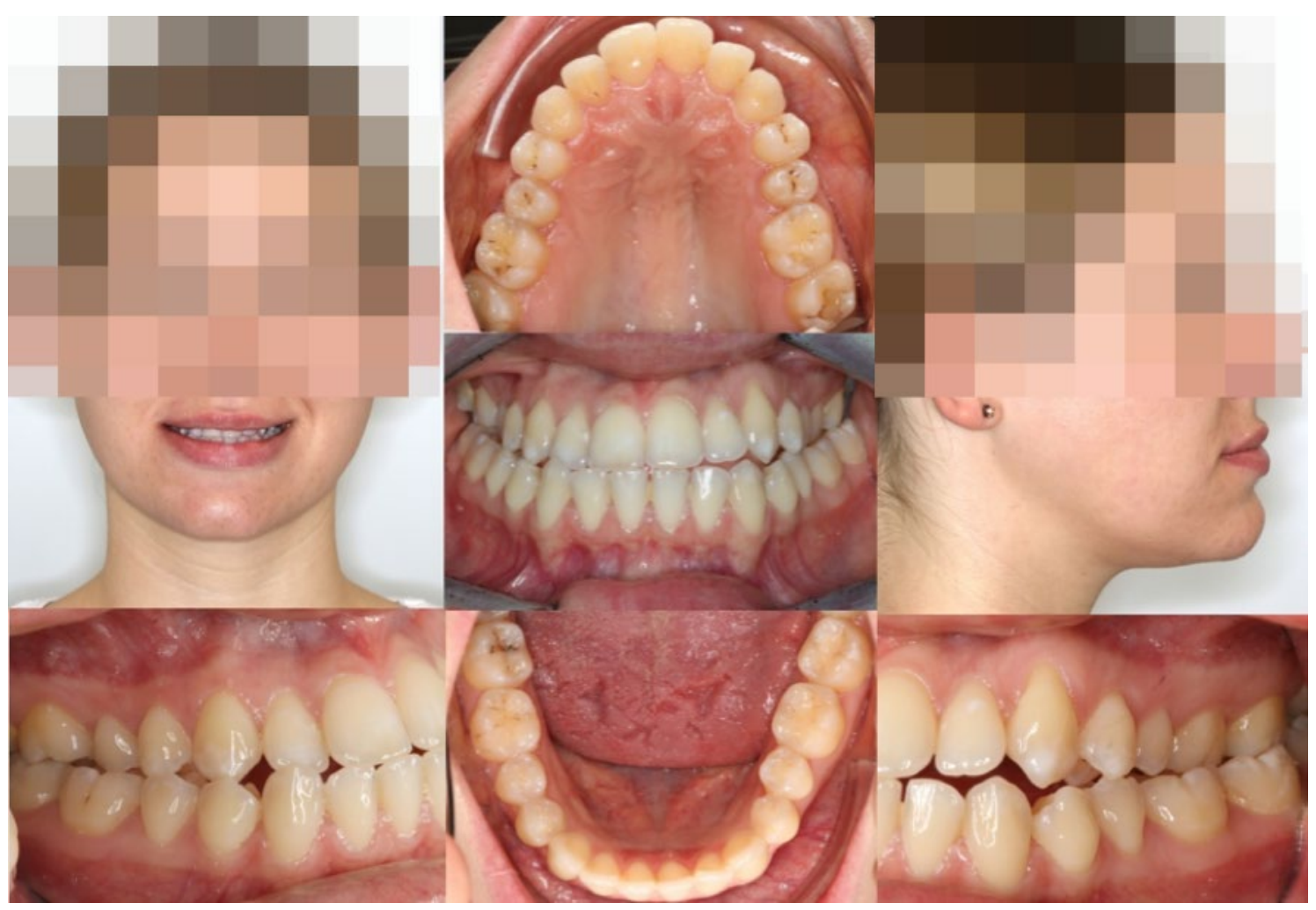

Abbildung 6: Anfangsbefund der Patientin. Extraoral dominiert die Laterognathie und die negative Lippentreppe bei einer ausgeprägten Kinnpartie. Intraoral zeigt sich eine schmale Maxilla mit stark protrudierten Frontzähnen und ein zirkulärer Kreuz-, und Kopfbiss.

Je nach skelettaler und dentaler Anomalie gestaltet sich die kieferorthopädische Vorbehandlung unterschiedlich (Abbildung 7). In dem hier gezeigten Patientenfall war, bedingt durch das starke transversale Defizit des Oberkiefers, eine chirurgisch unterstützte Gaumennahterweiterung im Vorfeld notwendig. Hierzu wurde eine Le-Fort 1 Osteotomie mit zusätzlicher Spaltung der Sutura palatina durchgeführt und ein skelettal fixierter Disktraktor eingebracht. Der Disktraktor wird postoperativ vom Patienten selbstständig an einer Schraube zweimal täglich aufgedreht und erweitert somit die Maxilla. Nachdem die geplante Breite erreicht wurde, wurde der Disktraktor durch einen Transpalatinalbogen ersetzt und mit der Multiband-Therapie begonnen.

Nach Abschluss der kieferorthopädischen Vorbehandlung erfolgt die Operationsvorbereitung. 


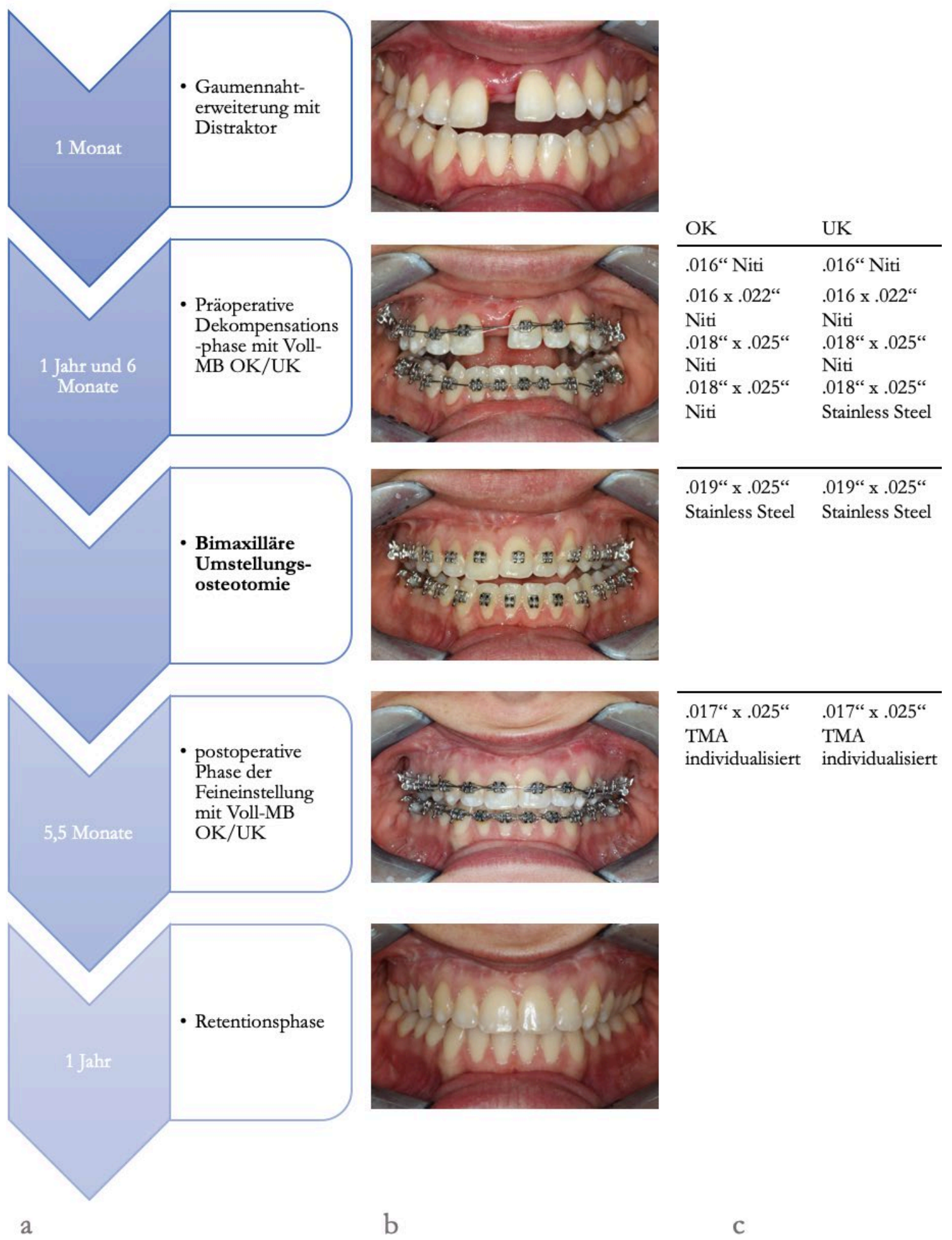

Abbildung 7: Darstellung des Therapieverlaufes a) schematische Darstellung der Therapieabschnitt und zeitlicher Ablauf des Patientenfalles b) intraorale Frontalansicht der Therapieabschnitte c) Bogenfolge der Multi-bandapparatur während der Therapieabschnitte (Niti= Nickel-Titan; TMA =Titan-Molybdän-Alloy). Nach der Entfernung der Multibandbehandlung erfolgt immer eine einjährige Retentionsphase mit herausnehmbaren Geräten, deren Tragezeit kontinuierlich reduziert wird. 


\subsubsection{Operationsplanung, Durchführung und postoperative Behandlung}

An der Universitätsmedizin Göttingen hat sich eine gelenkprotektive Operationsmethodik seit den 80er und 90er Jahren als fester Bestandteil der kieferorthopädischkieferchirurgischen Therapie etabliert (Luhr 1989; Schwestka-Polly 2004). Seither wurde das Konzept kontinuierlich optimiert und im Rahmen der Digitalisierung der Operationsplanung an das virtuelle Planungskonzept adaptiert. Eine gelenkbezügliche Therapie sieht, wie unter 1.3 beschrieben, den Erhalt der zentrischen Kondylenposition über den chirurgischen Eingriff hinweg vor (Schwestka-Polly 2004).

Hierzu erfolgt die Operationsplanung nach einem standardisierten Protokoll. Vom kieferorthopädischen Behandler der UMG werden Alginatabdrücke des Ober- und Unterkieferzahnbogens (Tetrachrom, Kaniedenta, Herford, Germany) und ein Zentrikregistrat genommen (weitere ausführliche Erläuterungen zur klinischen Anwendung befinden sich im Methodikteil dieser Studie). Das Zentrikregistrat am Patienten dient der Positionierung der Kondylen während der digitalen Volumentomografie (DVT). Zusätzlich $\mathrm{zu}$ einer intraoralen und extraoralen Fotoserie wird ein dreidimensionales Foto aufgenommen, welches zur Weichgewebssimulation der Operation genutzt wird. Während des dreidimensionalen Fotos sitzt der Patient aufrecht mit geschlossenem Mund, das Zentrikregistrat befindet sich in situ.

Die Planung der Operation erfolgt vollständig digital. Hierfür wird ein dreidimensionales Schädelmodell auf Basis des DVT-Datensatzes erstellt, die Gipsmodelle digitalisiert und mit Maxilla bzw. Mandibula kombiniert. Die Zielokklusion wird im Vorfeld einartikuliert und ebenfalls digitalisiert (Abbildung 8). Zum Einsatz kommen die medizinischen Software Mimics ${ }^{\circledR}$ InPrint 3.0 (Materialise, Belgien) und ProPlan ${ }^{\circledR}$ CMF 3.0 (DePuy Synthes CMF; Companie of Johnson \& Johnson). Das dreidimensionale Foto wird im Anschluss mit der skelettalen Grundlage aligned.

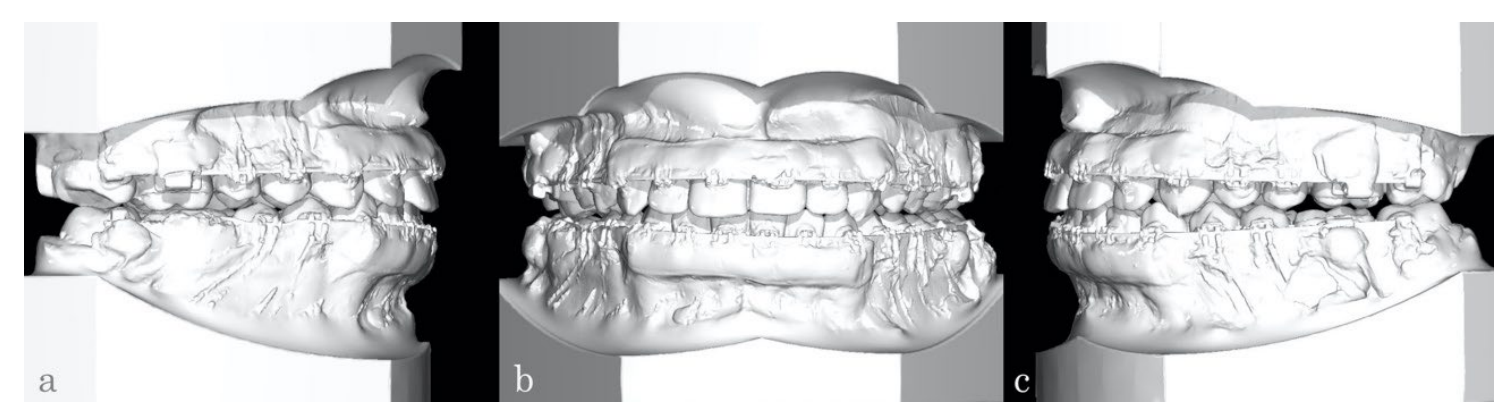

Abbildung 8: Digitalisierte Zielokklusion betrachtet von a) Patientenrechts b) Frontal c) Patientenlinks.

Zur Simulation der Operationsstrecken ist ein Schneiden der dreidimensionalen Modelle in einzelne Segmente notwendig. Orientiert wird sich hierbei an der Operationsschnittführung 
der LeFort-1 Osteotomie und der bilateralen sagittalen Spaltosteotomie nach Obwegeser/Dal-Pont (Dal Pont 1961; Obwegeser 1964) (Abbildung 9).

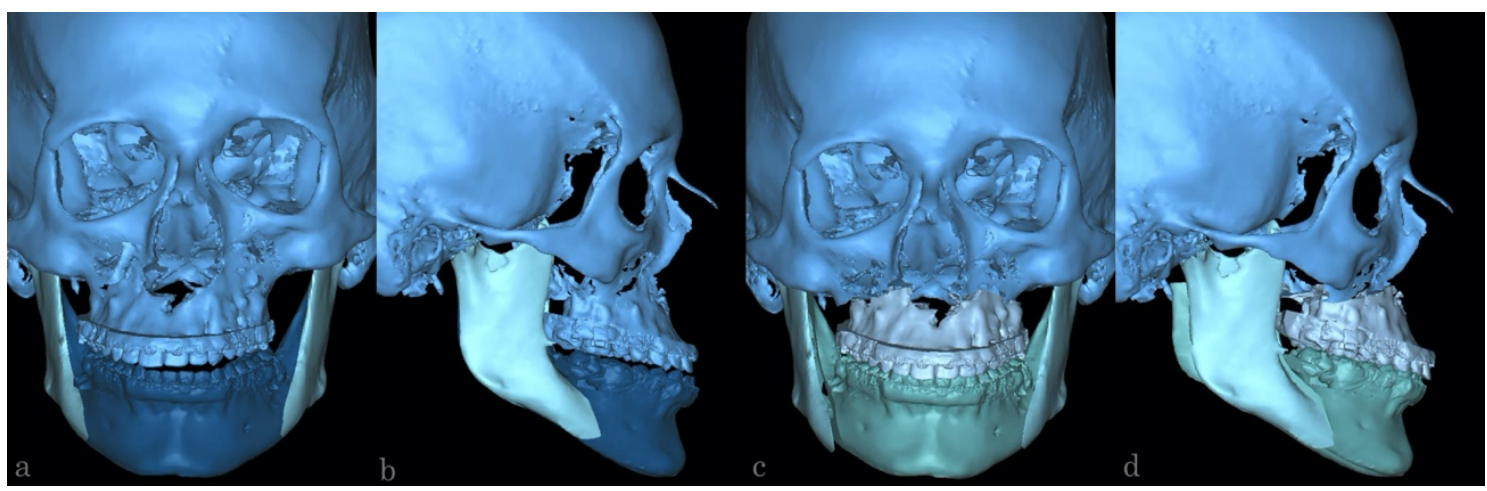

Abbildung 9: Dreidimensionale Rekonstruktion a) frontale präoperative Situation b) sagittale präoperative Situation c) frontale postoperative Situation und d) sagittale postoperative Situation.

Die Verlagerungsstrecken der einzelnen Kiefersegmente werden vom kieferorthopädischen Behandler zunächst festgelegt. Im Rahmen der interdisziplinären Operationsbesprechungen erfolgt im Anschluß die Vorstellung des Patientenfalles in der Mund-, Kiefer-, Gesichtschirurgie. Dabei erfolgt die Feineinstellung der Operationsstrecken mit zusätzlicher Weichteilsimulation. Durch die Verwendung der Software ProPlan ${ }^{\circledR}$ CMF 3.0 kann ein Eindruck des postoperativen Erscheinungsbildes des Patienten gewonnen werden (Abbildung 10).

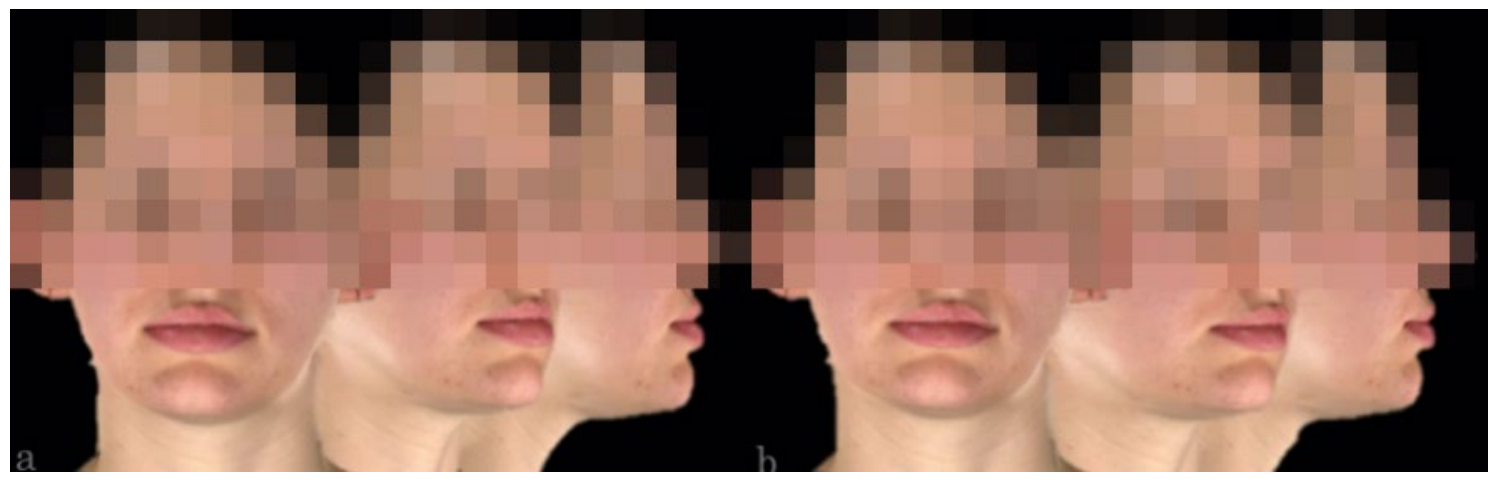

Abbildung 10: Weichteilsimulation a) präoperativ und b) postoperativ.

Entsprechend der früheren Planungen angelehnt an das System von Schwestka et al. (1990) und Luhr et al. (1986) werden die Operationssplinte digital erstellt.

Die zentrische präoperative Kondylenposition wird in den ersten Splint (Zentriksplint) überführt. Das Design erfolgt vollständig digital (Abbildung 11). Je nach Operationsmethode werden unterschiedliche viele Splinte angefertigt. Bei einer bimaxillären Umstellungsosteotomie werden 4 Splinte produziert: der erwähnte Zentriksplint, der Splint 
zur Positionierung der Maxilla (der sogenannte Intermediate Splint), der Splint zur Positionierung der Mandibula und ein finaler Splint (Übungssplint) zur postoperativen Sicherung der Situation (Abbildung 12). Der finale Splint verbleibt postoperativ für die nächsten Tage in situ und fungiert als eine Art funktionelles kieferorthopädisches Gerät und ermöglicht das Erlernen der neuen muskulären Position (Luhr et al. 1991). Konzipiert werden die Splinte in der Software ProPlan ${ }^{\circledR}$ CMF 3.0 und mithilfe eines für medizinische Zwecke zugelassenen Stereolithographie-Druckers der Firma Formlabs GmbH (Berlin, Germany) gedruckt. Verwendet werden die zahnmedizinischen Kunstharze Dental SG ${ }^{\circledR}$ und Dental LT® der Firma Formlabs.

Um bei alleiniger sagittaler Spaltung des Unterkiefers den proximalen Teil nicht an der Maxilla fixieren zu müssen, schlugen Luhr et al. (1991) einen Doppelimpressionensplint mit Balkonen im Bereich der Oberkiefermolaren vor. Intraoperativ kann so der gelenktragende Teil mit einer Osteosyntheseplatte am Balkon des Splintes immobilisiert werden und die konstante Position des Gelenkes sichergestellt werden.

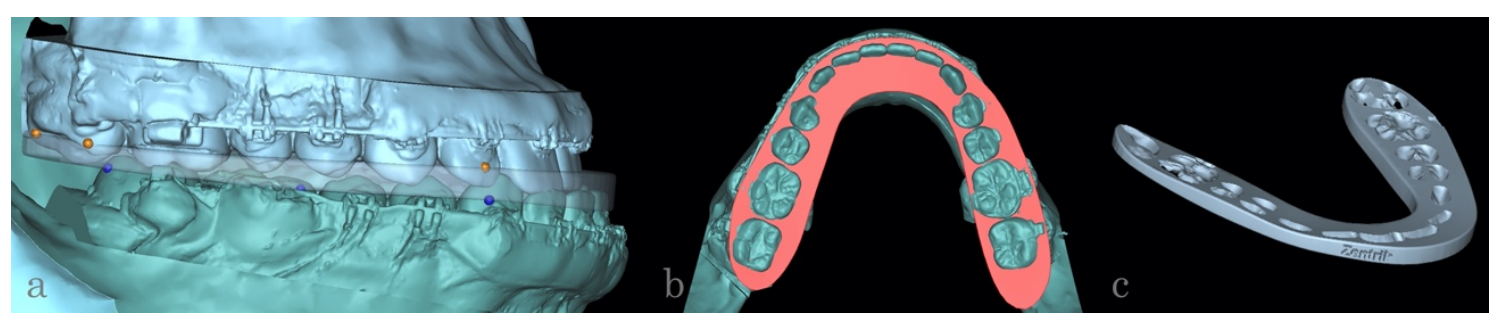

Abbildung 11: Workflow des Splintdesigns a) vestibuläre Begrenzungspunkte zur Definition der Splinthöhe b) transversale Ausdehnung c) fertiges Design.

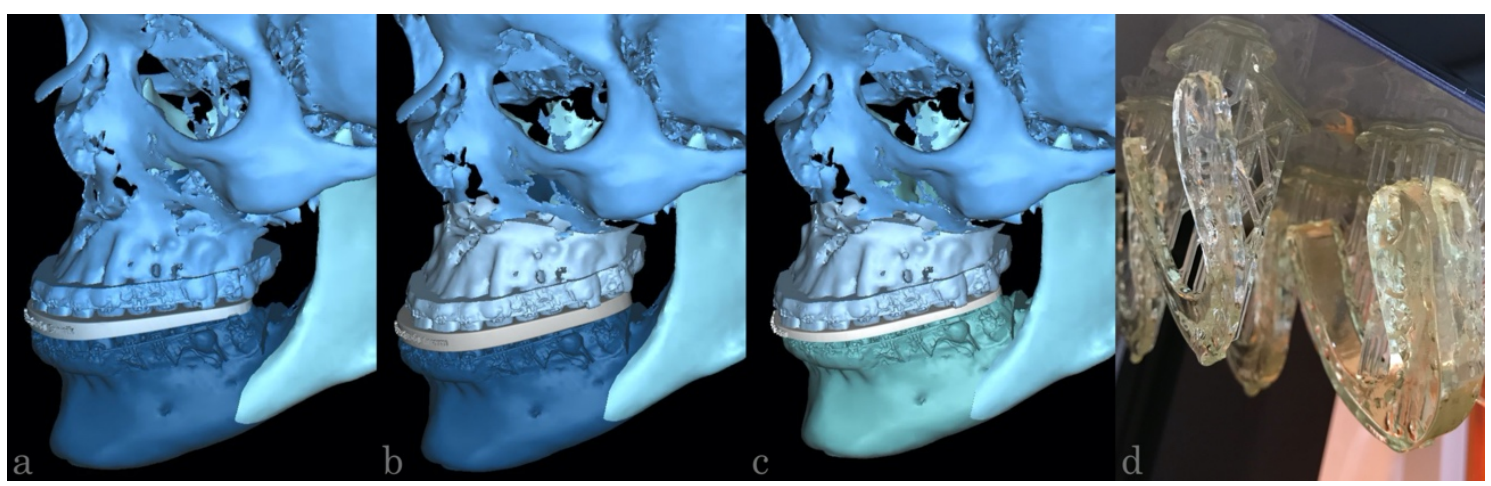

Abbildung 12: Splintdesign a) Zentriksplint in präoperativer Situation b) Intermediate Splint nach Oberkieferverlagerung c) Finalsplint nach Verlagerung der Mandibula d) Stereolithigraphie-Druck der Splinte.

Am Tag der stationären Aufnahme werden die Splinte intraoral angepasst und sterilisiert. Intraoperativ werden nach subperiostaler Präparation des Kieferwinkels, zunächst die Kiefer intermaxillär im Zentriksplint immobilisiert. Hierzu werden Drahtligaturen von der 
maxillären zur mandibulären Multibracketapparatur verdrillt. Der gelenktragende Teil wird nun mit Hilfe einer Osteosyntheseplatte oberhalb der Le Fort-1 an der Maxilla bzw. am Os zygomaticum oder am Doppelimpressionensplint fixiert (Abbildung 13).

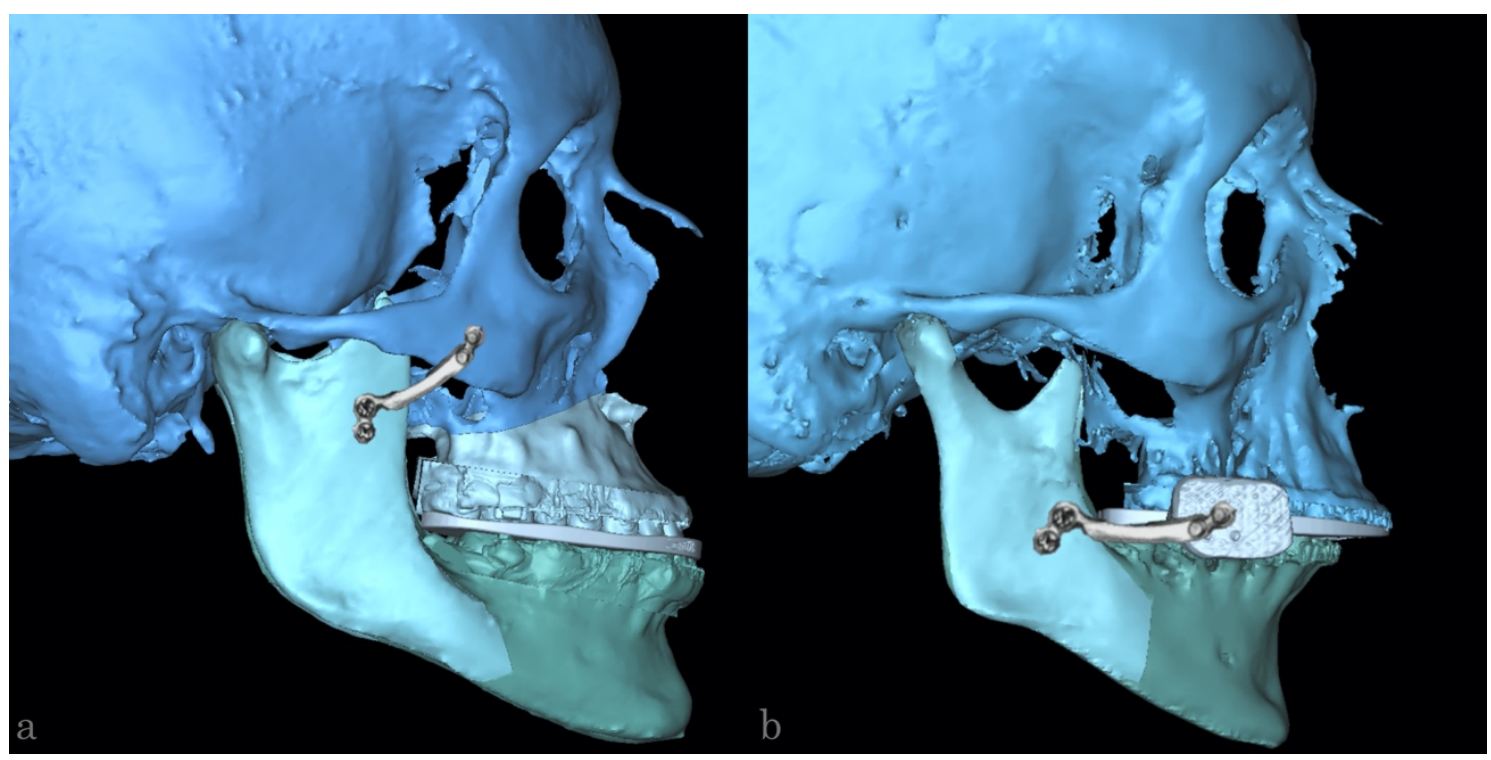

Abbildung 13: Darstellung der Fixierung des gelenktragenden Anteils bei a) bimaxillärer Umstellungsosteotomie und b) alleiniger sagittaler Spaltung des Unterkiefers.

Die Osteosyntheseplatte dient während der Verlagerung der Kiefer zur Sicherung der zentrischen Kondylenposition und wird erst nach finaler Verplattung beider Kiefer entfernt. Nach Entfernung des Zentriksplintes folgt bei einer bimaxillären Umstellung die „down fracture" des Oberkiefers entlang der Schnittebene der Le Fort-1-Osteotomie. Der Intermediate Splint wird intermaxillär verbunden und gibt die finale Position der Maxilla vor. Nach Verplattung der neuen Oberkieferposition wird der Intermediate Splint wieder entfernt.

Der Unterkiefer wird nach Obwegeser-Dal Pont beidseitig retromolar sagittal gespalten und über den Finalsplint in seine endgültige Position verschoben und intermaxillär immobilisiert. Nach der Versorgung der Mandibula mit funktionsstabilen Osteosyntheseplatten wird auch die Osteosyntheseplatte des proximalen Gelenkteiles entfernt. Die intermaxilläre Verdrahtung wird entfernt und durch Gummizüge ersetzt, der finale Splint verbleibt als Übungssplint in situ.

Auch die postoperative Nachsorge findet in der UMG interdisziplinär für alle Patienten statt. Bis zu drei Tage nach der Operation verbleibt der Übungssplint permanent im Mund (Abbildung 14). Anschließend wird der Splint postoperativ zusammen mit der Anomalie entsprechenden Gummizügen weitergetragen und vom Patienten selbstständig ein- und ausgegliedert. Die Tragezeit liegt bei 22-23 Stunden, der Splint wird nur zum Essen weicher Nahrung entfernt. 


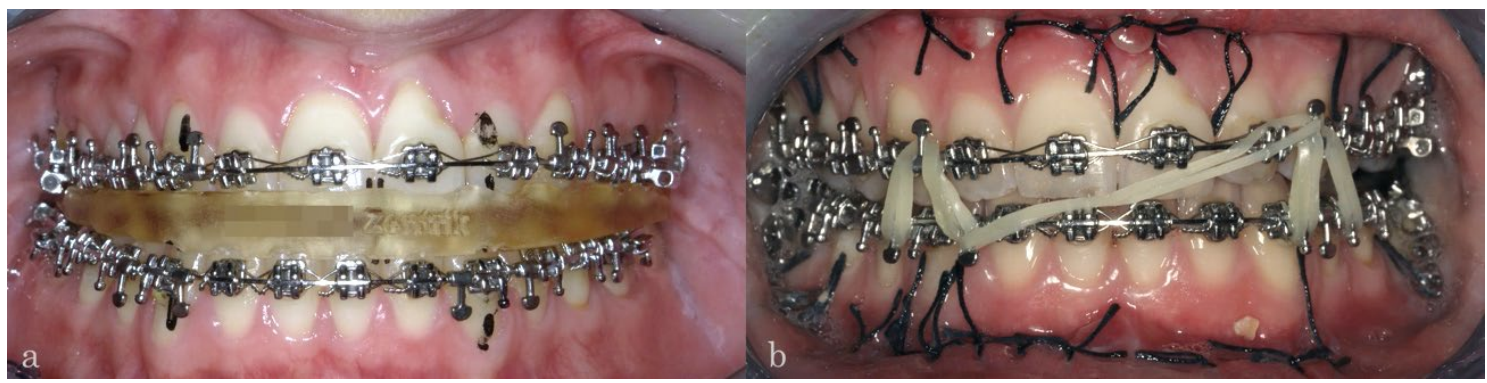

Abbildung 14: a) intraorale Passung eines Zentriksplintes b) einwöchige postoperative Situation mit intermaxillären Gummizügen, Nähte in Situ.

Mit der postoperativen kieferorthopädischen Feineinstellung sollte zeitnah begonnen werden, um die osseären Umbauprozesse zur Zahnbewegung optimal zu nutzen. Bis der Patient sicher in die Zielokklusion findet, kann der Übungssplint in Kombination mit intermaxillären Gummizügen getragen werden. Im Regelfall kann ca. 2 Wochen postoperativ der Splint endgültig herausgenommen werden, während das Ergebnis weiter über Gummizüge gesichert wird. So kann sich besonders im Bereich der Molaren und Prämolaren zeitnah eine sichere Okklusion einstellen und der Patient langsam die Mundöffnung trainieren. Patienten profitieren dazu sehr von postoperativer Lymphdrainage um den Rückgang der Schwellung zu beschleunigen. Der Zeitpunkt hierfür kann individuell bestimmt werden, laut Yaedú et al. (2017) sorgt die Therapie ab dem zweiten postoperativen Tag für ein signifikant schnelleres Abschwellen.

Die postoperative kieferorthopädische Feineinstellung sollte möglichst innerhalb von sechs Monaten abgeschlossen werden (Dowling et al. 1999), da die Motivation der Patienten nach der chirurgischen Intervention deutlich abnimmt. Ziel sollten sichere okklusale Verhältnisse im Sinne der „six keys of occlusion"(Andrews 1972) sein (Abbildung 15). 


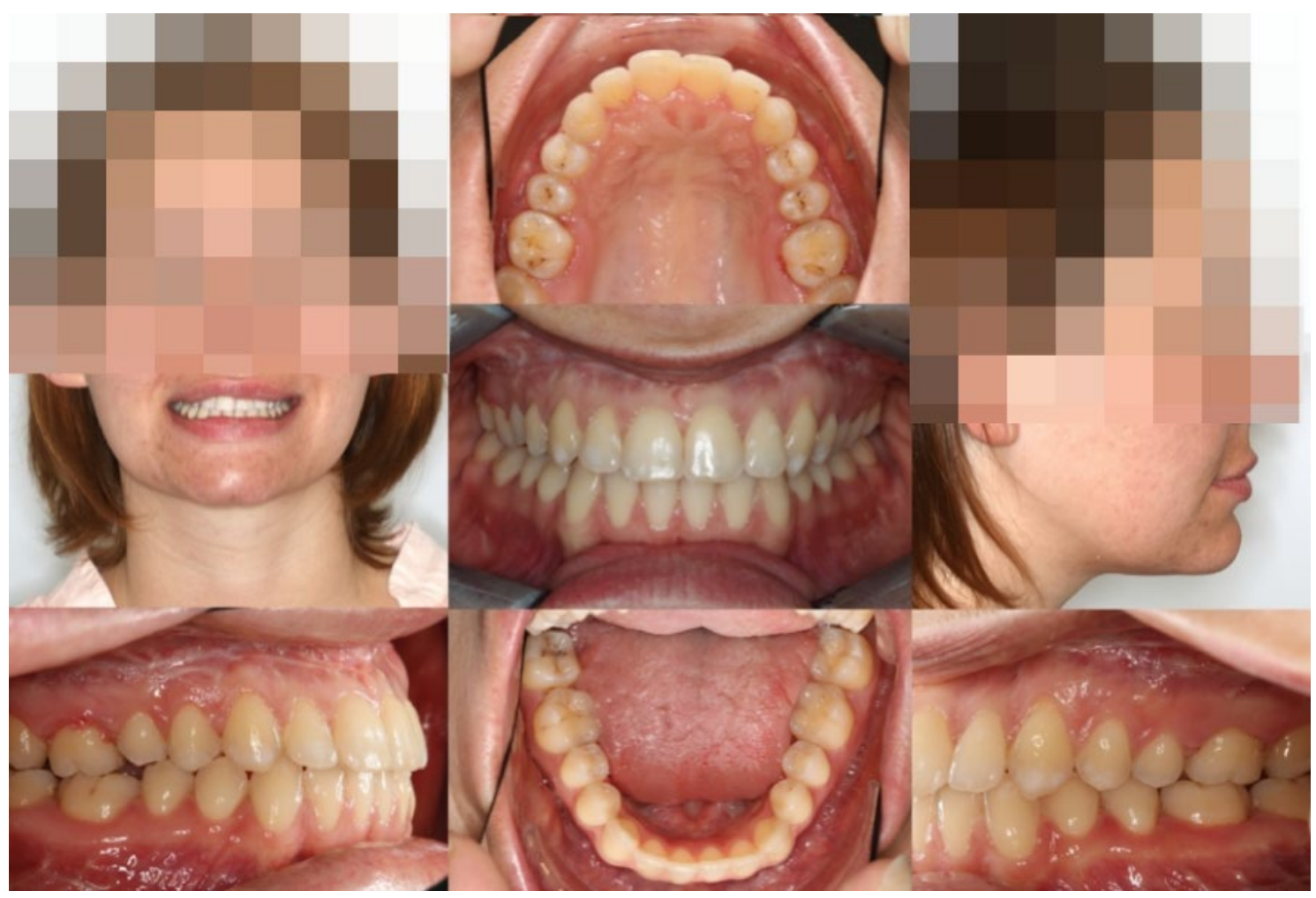

Abbildung 15: Befund nach abgeschlossener Therapie. Extraoral zeigt sich ein harmonisches und symmetrisches Erscheinungsbild. Auf dentaler Ebene wurde eine Angle-Klasse I mit einem physiologischen Overjet und Overbite erreicht. Im Vestibulum ist der Narbenzug zu erkennen.

\subsection{Ziel der Untersuchung}

Auf Basis der Literatur war das Ziel der vorliegenden Studie die Untersuchung des Einflusses der Generalanästhesie auf die zentrische Kondylenposition bei Dysgnathiepatienten. Bisherigen Untersuchungen basieren auf der Analyse von okklusalen Veränderungen, um Rückschlüsse auf die Kondylenposition zu schließen. Die Auswirkungen okklusaler Abweichungen auf kondylärer Ebene können mit dieser Methode auf Basis einartikulierter Gipsmodelle jedoch nicht festgestellt werden. Zusätzlich sind die Analysen nur mit zweidimensionalen linearen Verfahren beschrieben worden (Bamber et al. 1999; Reyneke und Ferretti 2002; Yaghmaei et al. 2013; Zak et al. 1992). Die hier vorliegende Studie zeigt eine neue Möglichkeit in der Dysgnathietherapie, die Analyse der Kondylenposition direkt auf Höhe der Gelenkfossa am Patienten zu erfassen. Zusätzlich werden zweidimensionale lineare als auch volumetrische dreidimensionale Messungen der Kondylenpositionen präund intraoperativ ermöglicht. Die vorliegende Studie leistet durch die Untersuchung der intraoperativen zentrischen Kondylenpositionierung damit einen Beitrag zur Evaluation der intraoperativen Freihandpositionierung der Gelenke und inwiefern sich diese von der präoperativen Zentrik unterscheidet. 
Folgende Fragestellungen sollten beantwortet werden:

1. Unterscheidet sich die zentrische Kondylenposition präoperativ am wachen Patienten mit aktivem Muskeltonus von der intraoperativen Position im relaxierten Zustand?

2. Finden sich auf dentaler und kondylärer Ebene vergleichbare Abweichungen zwischen der prä- und intraoperativ registrierten Zentrik?

3. Eignet sich eine volumetrische dreidimensionale Analyse besser als lineare cephalometrische Messungen zur Detektion von Veränderungen der Kondylenposition?

4. Unterscheidet sich die prä- und intraoperative Zentrik in Abhängigkeit der skelettalen Klasse?

5. Beeinflusst eine Laterognathie die intraoperative Reproduzierbarkeit der Zentrik? 


\section{Material und Methoden}

\subsection{Patientenkollektiv und Rekrutierung}

Das Probandenkollektiv dieser einarmigen, prospektiven und monozentrischen Studie umfasste 30 gesunde, erwachsene Patienten (weiblich: $n=16$; männlich $n=14$ ) im Alter zwischen 18 und 49 Jahren (Mittelwert $\mathrm{M}=$ 25,8; Standardabweichung SD=7,1), bei denen eine ausgeprägte skelettale Dysgnathie mit Indikation zur kombiniert kieferorthopädischkieferchirurgischen Therapie (mindestens Grad 4 nach Index of orthognathic funktional treatment need (Ireland et al. 2014)) vorlag. Alle Patienten unterzogen sich im Zentrum für Zahn-, Mund-, Kieferheilkunde der Universitätsmedizin Göttingen dem Wahleingriff einer orthognathen Chirurgie.

Die Patientenrekrutierung erfolgte zwischen Juni 2018 und Mai 2019 nach Zustimmung der klinikeigenen Ethikkommission (Nr.16/4/18) in Übereinstimmung mit der Deklaration von Helsinki. Alle Patientin nahmen freiwillig an der Studie teil und gaben ihr schriftliches Einverständnis, das jeder Zeit widerrufbar ist.

Folgende Bedingungen führten zum Studienausschluss:

- Ausgeschlossen von der Studie wurden Patienten mit akuten craniomandibulären Symptomen oder funktionellen Einschränkungen der Kiefergelenke

- $\quad$ einem erhöhten Narkoserisiko (ASA>3 gemäß (Anesthesiologists 2014),

- schwerbehandelbaren Infektionserkrankungen (z. B. HIV, Hepatitis C), syndromalen Kiefer- und Schädelanomalien oder Lippen-Kiefer-Gaumenspalten.

Die Fallzahl von 30 Probanden wurde in G*Power (v.3.1.9.2, Universität Düsseldorf) für die Anwendung eines Wilcoxon-Vorzeichen-Rang-Tests für verbundene Stichproben, unter Voraussetzung eines Signifikanzniveaus von 0,05, einer Power von 0,9 sowie einer DropOut Quote von 10\% berechnet. Die Effektstärke wurde basierend auf einer Magnetresonanz-Tomographie-Untersuchung von (Kandasamy et al. 2013) zur Variation der Kondylenposition in superior-inferiorer Richtung geschätzt (Mittelwert-Differenz: 0,26 ; SD-Differenz: 0,43).

\subsection{Einteilung des Patientenkollektives}

Das Patientenkollektiv wurde anhand der cephalometrische Auswertung des DVTs in skelettale Klassen eingeteilt (Tabelle 1), auf Basis der skelettalen Relation nach Wits (Jacobson 1975, 1976)

- Patientin mit einem Wits $=0 \pm 2 \mathrm{~mm}$ wurden als Klasse I klassifiziert

- Patienten mit einem Wits $>+2 \mathrm{~mm}$ wurden als Klasse II klassifiziert

- Patienten mit einem Wits $<-2 \mathrm{~mm}$ wurden als Klasse III klassifiziert 
Des Weiteren wurden die Patienten anhand ihrer Symmetrie klassifiziert. Hierfür wurde die Mentondeviation zur Sagittalebene in $\mathrm{mm}$ anhand der dreidimensional erstellten Modelle vermessen und die Patienten in zwei Gruppen unterteilt:

- Symmetrische Patienten mit einer Mentondeviation $<4 \mathrm{~mm}$

- Asymmetrische Patienten mit einer Mentondeviation $>4 \mathrm{~mm}$

Der Richtwert von 4mm zur Definition einer Laterognathie wurde gemäß des von Kim et al. (2014) beschriebenen Vorschlags zur Klassifizierung von Gesichtsasymmetrien übernommen.

Tabelle 1: Patienten und Subklassifikation

\begin{tabular}{|c|c|}
\hline Patientenkollektiv & $\mathrm{n}=\mathbf{3 0}$ \\
\hline Männlich & $\mathrm{n}=14$ \\
\hline Weiblich & $\mathrm{n}=16$ \\
\hline \multirow[t]{2}{*}{ Patientenalter (Jahren) } & 18-49 Jahre \\
\hline & $\mathrm{M}=25,8 ; \mathrm{SD}=7,1$ \\
\hline \multicolumn{2}{|l|}{ Skelettale Klasse der Patienten nach WITS in mm } \\
\hline skelettale Klasse I (Wits $=0 \pm 2 \mathrm{~mm})$ & $\mathrm{n}=3(\mathrm{M}=0,7 ; \mathrm{SD}=1,7)$ \\
\hline skelettale Klasse II (Wits > + 2mm) & $\mathrm{n}=13(\mathrm{M}=7,5 ; \mathrm{SD}=3,8)$ \\
\hline skelettale Klasse III (Wits $<-2 \mathrm{~mm}$ ) & $\mathrm{n}=14(\mathrm{M}=-7,0 ; \mathrm{SD}=3,2)$ \\
\hline \multicolumn{2}{|c|}{ Patienten aufgeteilt bzgl. ihrer Asymmetrie (Mentondeviation in mm) } \\
\hline symmetrische Patienten (Mentondeviation $<4 \mathrm{~mm}$ ) & $\mathrm{n}=20(\mathrm{M}=1,3 ; \mathrm{SD}=1,1)$ \\
\hline asymmetrische Patienten (Mentondeviation $>4 \mathrm{~mm}$ ) & $\mathrm{n}=10(\mathrm{M}=6,1 ; \mathrm{SD}=1,5)$ \\
\hline \multicolumn{2}{|l|}{ Patienten aufgeteilt bzgl. des chirurgischen Vorgehens } \\
\hline Le Fort 1 und BSSO & $\mathrm{n}=22$ \\
\hline Le Fort 1 & $\mathrm{n}=2$ \\
\hline BSSO & $\mathrm{n}=6$ \\
\hline Anzahl der Zentrikregistrate & $\mathrm{n}=60$ \\
\hline Anzahl der vermessenen Kondylenpositionen & $\mathrm{n}=120$ \\
\hline
\end{tabular}

(n steht für die Anzahl der untersuchten Patienten, M für den Mittelwert und SD für die Standardabweichung) 


\subsection{Studienablauf}

\subsubsection{Klinische Funktionsanalyse}

Um Patienten im Vorfeld mit einer akuter Craniomandibulären Dysfunktion von der Studie auszuschließen (siehe Ausschlusskriterien Punkt 3.1), fand im Vorfeld eine klinische Funktionsanalyse statt. Diese beinhaltete eine spezielle Schmerzanamnese, die Palpation der Kaumuskulatur, die klinische Überprüfung der okklusalen Verhältnisse und der Kiefergrenzbewegungen. Zusätzlich wurde das Kiefergelenk auf Druckdolenzen und Gelenkgeräusche überprüft. Zum Einsatz kam der Befundbogen „klinischer Funktionsstatus“ der DGFDT (2020). Erst nach einer unauffälligen Analyse wurden die Patienten ins Probandenkollektiv aufgenommen.

\subsubsection{Bissnahme - Registrierung der zentrischen Kondylenposition}

Zum Vergleich der Kondylenpositionen präoperativ im wachen Zustand und intraoperativ im narkotisierten, muskelrelaxierten Zustand, wurden bei jedem Patienten zwei Kieferrelationsbestimmungen mithilfe eines okklusalen Registrates durchgeführt -1 . Präoperativ während der OP-Vorbereitung und 2. Intraoperativ nach erfolgter Anästhesie. Das Registrat zur Bestimmung der zentrischen Kondylenposition (im Folgenden als Zentrikregistrat bezeichnet) wurde mittels Primobyte (Primodent, Bad Homburg, Deutschland), einem zahnmedizinisch zugelassenen, dimensionstreuen und biokompatiblen Werkstoff für intraorale, okklusale Registrate genommen. Primobyte besteht aus einer Kombination aus einer individuell anpassbaren Registrierplatte und einer intraoral zu verarbeitenden, hochviskösen Detailpaste. Beide Bestandteile sind auf Basis eines lichthärtenden Kunststoffes.

Die Definition der zentrischen Kondylenposition richtete sich nach den Vorgaben der DGFDT: „die kranio-ventrale, nicht seitenverschobene Position beider Kondylen bei physiologischer Kondylus-Diskus-Relation und physiologischer Belastung der beteiligten Gewebestrukturen“ (DGZMK 2005).

Das erste Zentrikregistrat wurde im Rahmen der Operationsplanung am wachen Patienten angefertigt. Zur Herstellung der Gipsmodelle wurden zunächst Alginatabdrücke des Oberund Unterkiefers angefertigt (Tetrachrom, Kaniedenta, Herford, Germany). Anschließend wurde im zahntechnischen Labor die individuelle Registrierplatte anhand der in habitueller Interkuspidation einartikulierten Modelle hergestellt (Abbildung 16). Diese Registrierplatte wurde im Artikulator soweit ausgeschliffen, dass zunächst keine Bisssperrung mehr vorhanden war, um intraoral eine Veränderung der vertikalen Kieferrelation so gering wie möglich zu halten. Der Sitz der Registrierplatte wurde intraoral überprüft und korrigiert. Vier Markierungen, im Bereich der ersten Oberkiefermolaren und der Caninen, wurde für die Detailpaste auf die Registrierplatte gesetzt. Der Patient wurde für die Registrierung der 
zentrischen Kondylenposition mit leicht überstreckten Kopf horizontal auf dem Behandlungsstuhl gelagert (Abbildung 17).

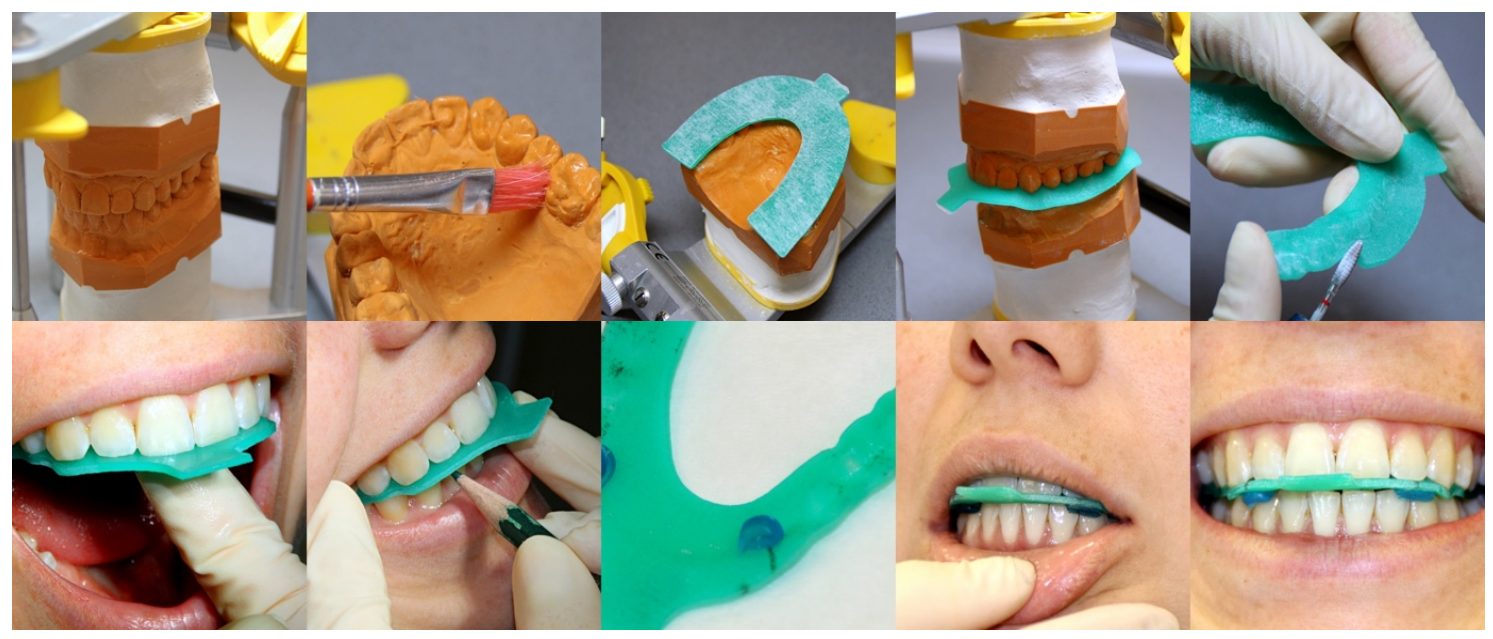

Abbildung 16: Herstellung und Bissnahme des Zentrikregistrates mit Primobyte.

Die Detailpaste wurde zunächst auf die beiden mesialen Markierungen aufgetragen, der Patient mittels Kinnführungsgriff in die gefühlte zentrische Kondylenposition geführt und das Material lichtpolymerisiert. Der Griff erfolgt in Anlehnung an den Kinnführungsgriff nach Lauritzen (Lauritzen 1974), jedoch passiv ohne Anwendung aktiver Kräfte. Der Unterkiefer wurde hierbei mithilfe des Daumens (mittige Positionierung unterhalb der Unterkieferinzisivi), Zeige- und Mittelfingers (jeweils lateral-caudal des Unterkiefers) nach posterior und kranial bewegt und registriert (Abbildung 18). Der Patient musste die lockere Führung des Unterkiefers zulassen. Bei Patienten mit starker muskulärer oder emotionaler Anspannung wurden zunächst einige Unterkieferbewegungen zur Lockerung durchgeführt. Der Vorgang wurde für die distalen Markierungen wiederholt.

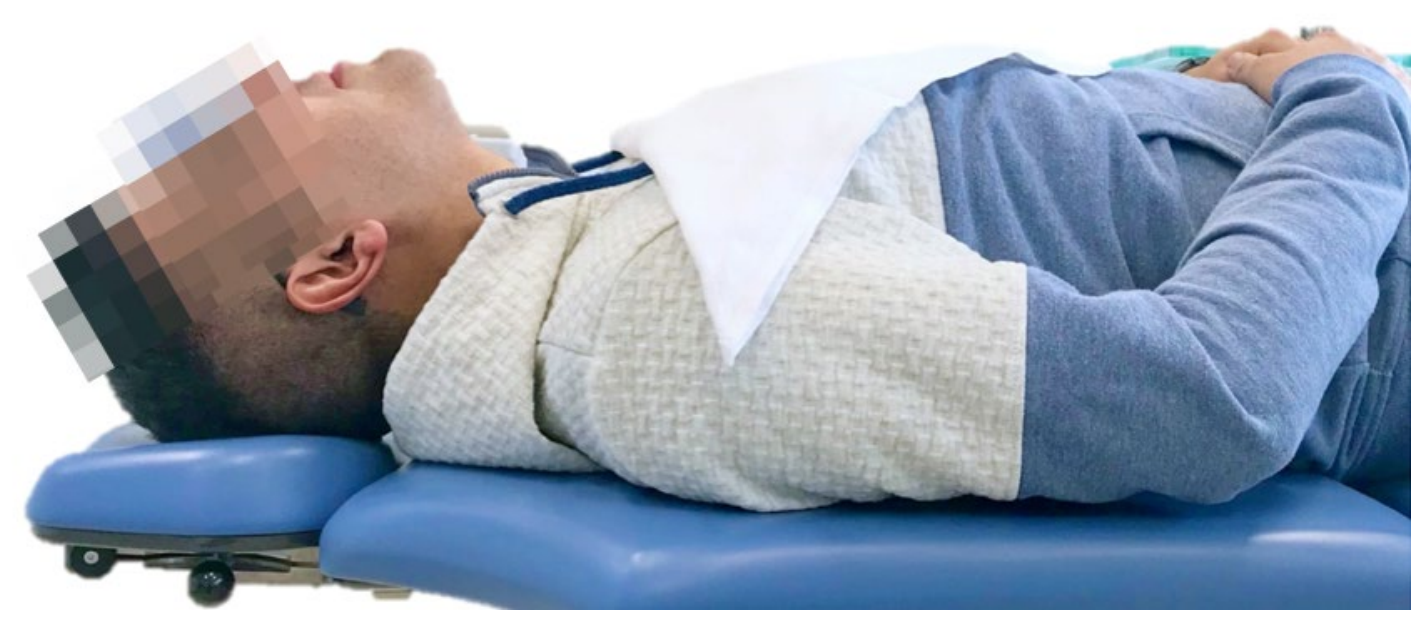

Abbildung 17: Lagerung des Patienten für die Zentriknahme. 


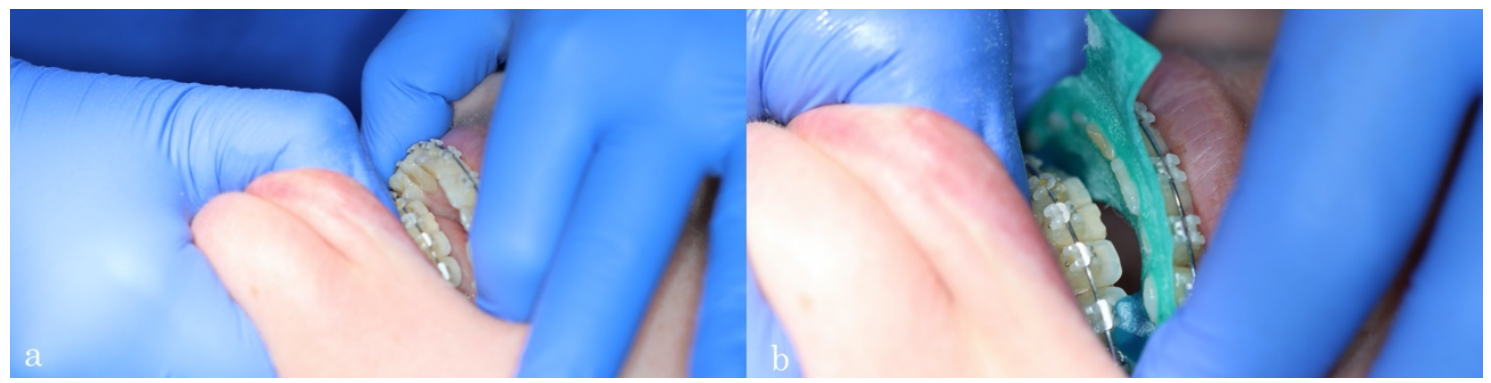

Abbildung 18: Lauritzenhandgriff a) "Übungsbewegung" mit dem Patienten. Der Behandler stützt den Unterkiefer mit Daumen im Bereich der Frontzähne und mit den Fingern im Bereich des Kinnes. Nach Entspannung des Patienten wird der Unterkiefer drucklos durch den Behandler geschlossen b) Wiederholung der Bewegung mit Primobyteregistrat. Die linke Hand des Behandlers fixiert die Registratplatte am Oberkiefer.

Die Herstellung dieses präoperativen Zentrikregistrates (Abbildung 19) ist Teil der regulären Operationsvorbereitung und dient sowohl zur Positionierung der Kondylen während der DVT als auch der Bestimmung der Kondylenposition während der Umstellungsosteotomie.

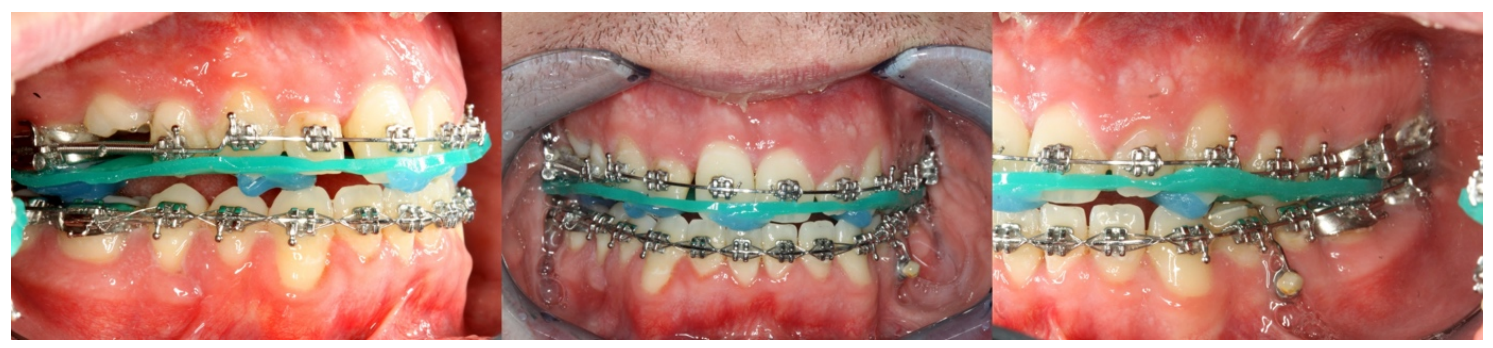

Abbildung 19: Primobyte Registrat in situ.

Im Rahmen dieser Studie erfolgte ein zweites, intraoperatives Zentrikregistrat am narkosierten und relaxierten Patienten. Die Vorbereitung im zahntechnischen Labor erfolgte analog.

Nach Einleitung der Vollnarkose wurde die zentrische Kondylenposition nach identischem Vorgehen genommen. Hierzu lag der nasalintubierte Patient horizontal auf dem Operationstisch, der Kopf wurde leicht überstreckt.

Die genutzten Narkotika und Relaxantien unterlagen keinen studienbedingten Vorgaben und wurden vom behandelnden Anästhesisten bestimmt. Zur Anwendung kamen Propofol, Remifentanil und Rocuronium.

Alle angefertigten Registrierungen erfolgten durch die Verfasserin dieser Arbeit um individuelle Schwankungen zu reduzieren. 


\subsubsection{Untersuchung der Registratdicke}

Zur Beurteilung des vertikalen Einflusses des Zentrikregistrates wurden alle 60 prä- und intraoperative Registrate an vier Messpunkten mit einem Tasterzirkel vermessen. Zur Vergleichbarkeit wurden die dünnsten Bereiche Regio der Zähne 16,13,23,26 ausgewählt.

\subsubsection{Bildgebung}

Im Rahmen der OP-Planung wurde eine DVT nach standardisiertem Protokoll mit Zentrikregistrat in situ durchgeführt (Orange Dental PaX Zenith 3D, Field of View 240x190 mm, Voxel Größe 0,3mm). Die errechneten Daten wurden als DICOM-Datensätze genutzt.

\subsubsection{Digitalisierung der Zentrikregistrate}

Um einen objektiven Vergleich der klinisch erhobenen prä- und intraoperativen Zentrikregistrate zu ermöglichen, erfolgte die Überführung beider klinischen Situationen und der analogen Situationsmodelle, in ein patientenindividuelles Schädelmodell auf Basis der zur OP-Planung angefertigten DICOM-Datensätze.

Initial wurde mithilfe der Software Mimics ${ }^{\circledR}$ InPrint 3.0 (Materialise, Belgien) ein dreidimensionales Modell anhand der Grauwerte der DVT rekonstruiert. Hierzu wurde zunächst eine region of interest (ROI) des gesamten Schädels auf Basis des thresholds hergestellt. Der threshold basiert auf der Hounsfield-Skala mit der die Abschwächung der Röntgenstrahlung in Graustufen beschrieben wird. Die Einheit ist hounsfield unit (HU) (Brooks 1977). Hierzu enthält das Programm Mimics ${ }^{\circledR}$ InPrint 3.0 ein tool um den individuell optimalen threshold einzustellen um sowohl kompakten als auch spongiösen Knochen darzustellen sowie Artefakte und Weichgewebe herauszurechnen (im Durchschnitt 226HUMAX). Größere Artefakte oder Regionen, die für die Untersuchung irrelevant waren (z. B. Wirbelsäule, Zungenbein), wurden aus der ROI ausgeschnitten (Abbildung 20). 


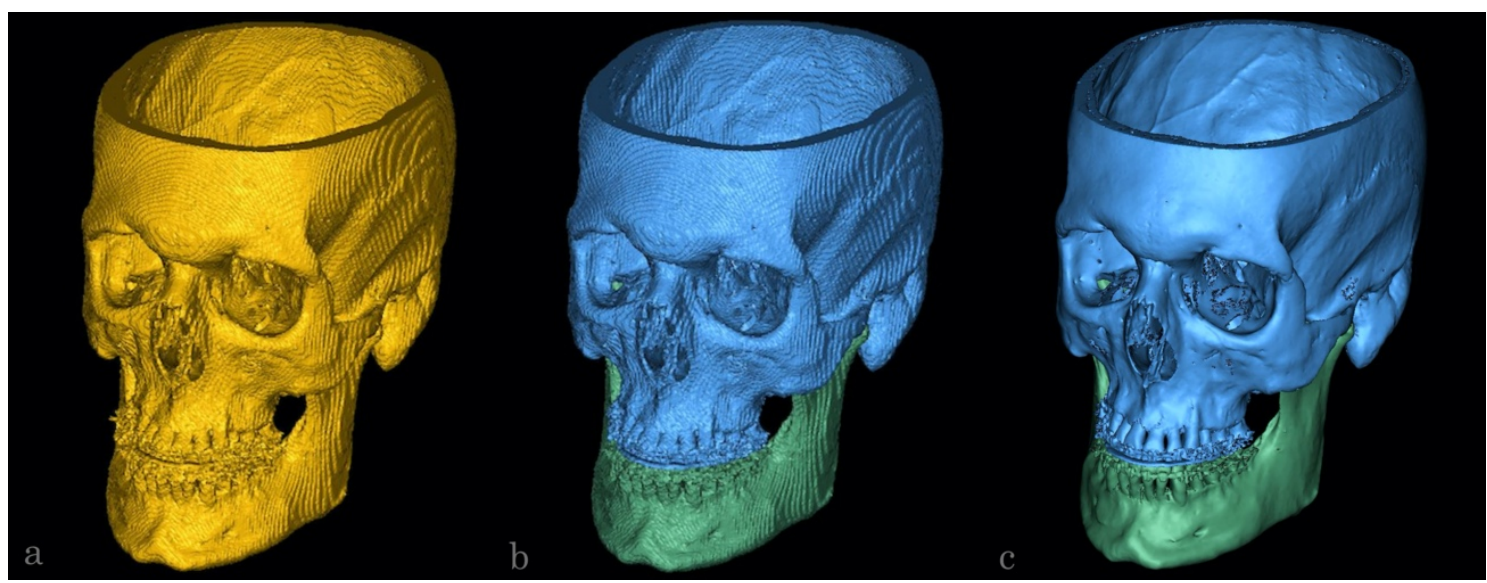

Abbildung 20: Rekonstruktion der Schädelmodelle a) nach Entfernung größerer Artefakte b) nach Nachbearbeitung und Trennung in Maxilla und Mandibula c) geglättetes Modell.

Das tool „Split“ in Mimics ${ }^{\circledR}$ InPrint 3.0 diente der Teilung der ROI in die Maxilla mit Cranium und in die Mandibula. Die Bereiche der Kondylen, die unterhalb des eingestellten thresholds liegen, wurden mithilfe der Option „3D Interpolate" und einem threshold im niedrigen Bereich, in Abhängigkeit der jeweiligen Knochendichte (teilweise $<50 \mathrm{HU}$ ) nachbearbeitet (Abbildung 21). Am erzeugten 3D Modell wurden Löcher unter $0,30 \mathrm{~mm}$ gefüllt und das Modell mit einer Detailgenauigkeit von 0,50 mm geglättet. Die Genauigkeit der Methodik wurde evaluiert indem ein zweiter Untersucher die Modelle erneut erstellte und die Modelle beider Untersucher miteinander überlagert wurden.

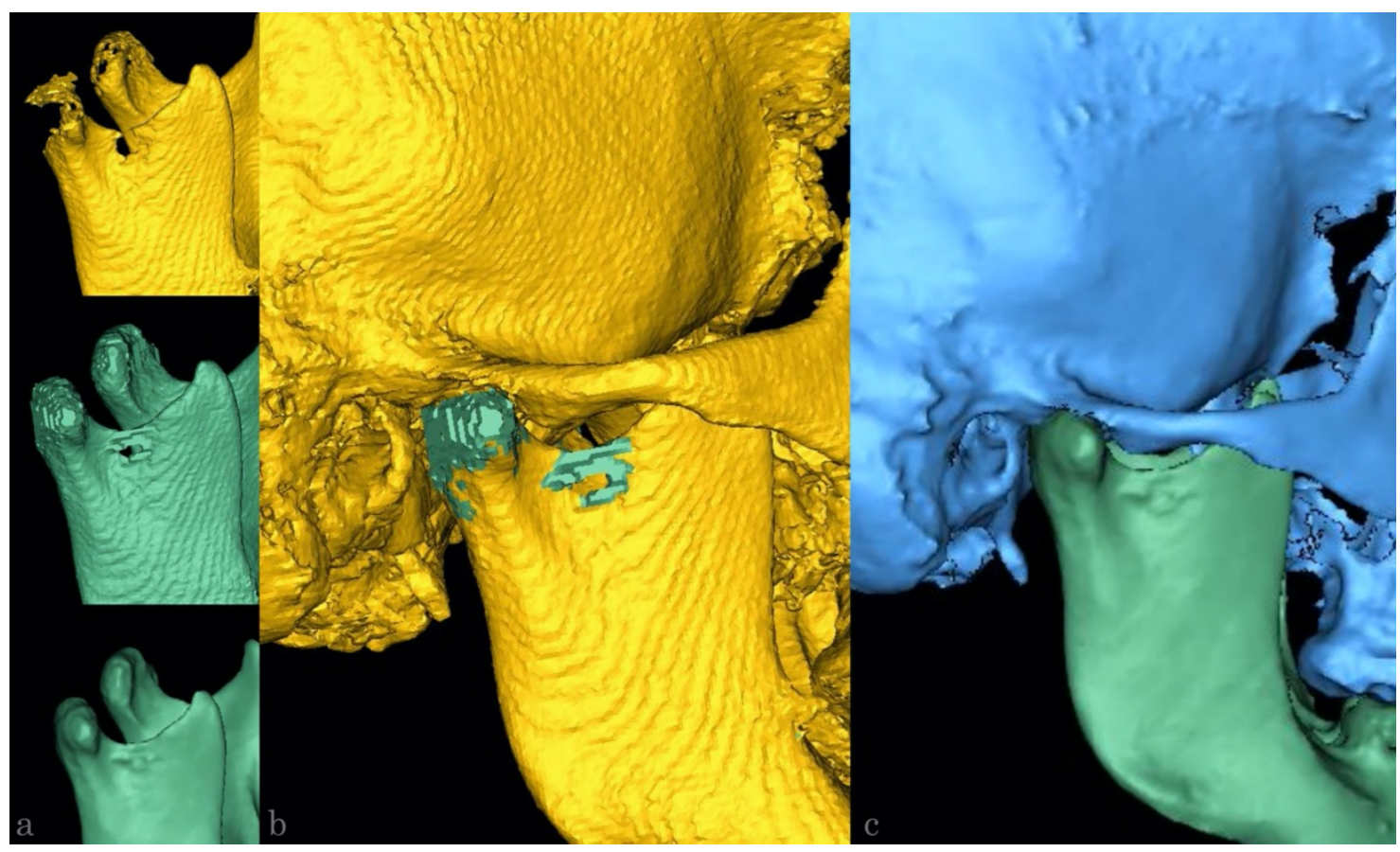

Abbildung 21: Rekonstruktion der Kondylen a) Oben vor der Bearbeitung mit einem individuellen threshold, Mitte nach der manuellen Bearbeitung und Unten nach der Glättung b) Überlagerung der bearbeiteten (grün) und nicht bearbeiteten (gelb) Bereiche c) endgültiges geglättetes Modell. 
Für eine exaktere Darstellung der dentalen Okklusionsbeziehungen wurden die analogen Gipsmodelle des Ober- und Unterkiefers mithilfe des S300 Ortho Scanners (Zirkonzahn, Gais, Italien) unter Einsatz der Primobyte-Registrate in prä- und intraoperativer Registrierung digitalisiert und als plain stl (reine Oberflächendatei als standart triangulation language) weiterverarbeitet (Abbildung 22). Hierzu wurden vier einzelne stl-Dateien hergestellt, eine Datei umfasst das Oberkiefer Gipsmodell, eine das Unterkiefer Gipsmodell und jeweils eine Datei die gescannte prä- und intraoperative Okklusion. Die Scanpräzision lag bei $\leq 10 \mu \mathrm{m}$. Laut Cho et al. (2015) findet sich bei dieser Scanpräzision kein signifikanter Unterschied zwischen digitalen und konventionellen Modellen bei nichtpräparierten Zähnen.

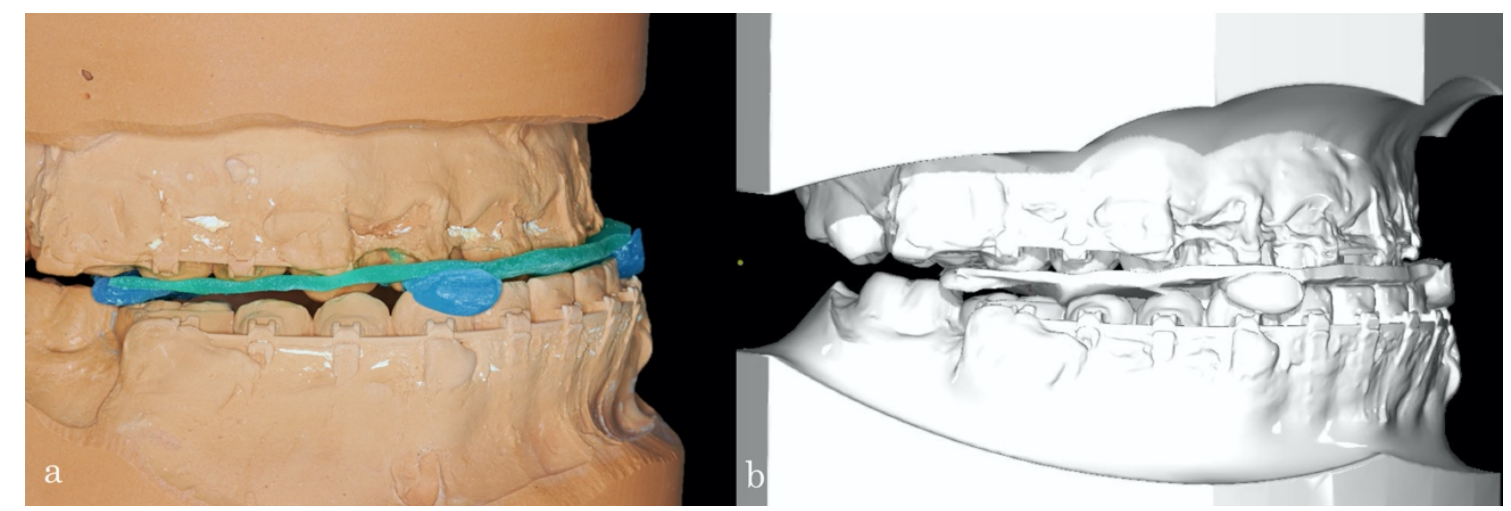

Abbildung 22: Modelle mit Primobyte-Registrat eines Patienten mit ausgeprägter skelettaler Klasse III a) analoges Gipsmodell b) digitalisiertes Modell.

Das dreidimensionale, in Mimics ( ${ }^{\circledR}$ InPrint 3.0 generierte und in Maxilla/Cranium und Mandibula geteilte Modell, wurde in die medizinische Software ProPlan ${ }^{\circledR}$ CMF 3.0 (DePuy Synthes CMF; Companie of Johnson \& Johnson) importiert und mit den stl-Dateien der jeweiligen gescannten Gipsmodelle kombiniert. Hierzu wurde das tool „Orthognathic Wizard“ zur Planung von Dysgnathiechirurgie genutzt. Diese zusammengesetzten Modelle (hier weiter als Composite-Modell bezeichnet) wurden jeweils für Oberkiefer mit Cranium und Unterkiefer hergestellt. Über drei korrespondierende Punkte (Abbildung 23a) wurden das dreidimensionale Schädelmodell und das digitalisierte Gipsmodell übereinander gelagert. Über das anschließende Alignment der gesamten Zahnoberflächen (Abbildung 23b-c) wurde eine exakte Übereinstimmung erreicht. Nach dem gleichen Vorgehen wurde separat das Composite-Modell des Unterkiefers hergestellt. 


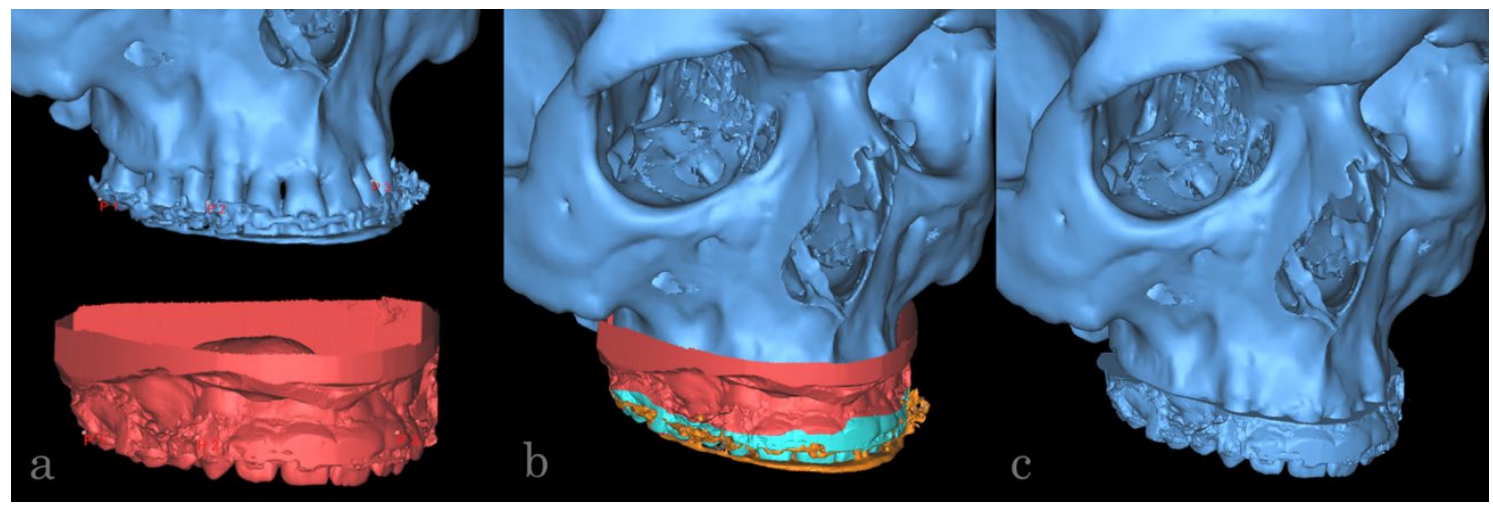

Abbildung 23: Herstellung des Composite-Modelles Maxilla/Cranium a) Markierung der korrespondierenden Punkte b) Alignment der Zahnoberflächen c) fertiges Composite-Modell.

Die okklusale Registrierung erfolgte nach demselben Schema wie die Herstellung der Composite-Modelle und wurde anhand korrespondierender Punkte und Alignment der gesamten Zahnoberfläche errechnet (Abbildung 24). Hierzu wurde die Funktion „Occlusion Registration" der Planungssoftware ProPlan ${ }^{\circledR}$ CMF 3.0 genutzt. Simuliert wurde eine „mandible only surgery“, bei der ausschließlich über die digitalisierte Okklusion das komplette Unterkiefer-Modell (fusionierte Mandibula inklusive Kondylen und gematchte Zahnoberfläche) in die gewünschte Position verschoben wurde. Das Composite-Modell der Maxilla wurde hierbei nicht bewegt.

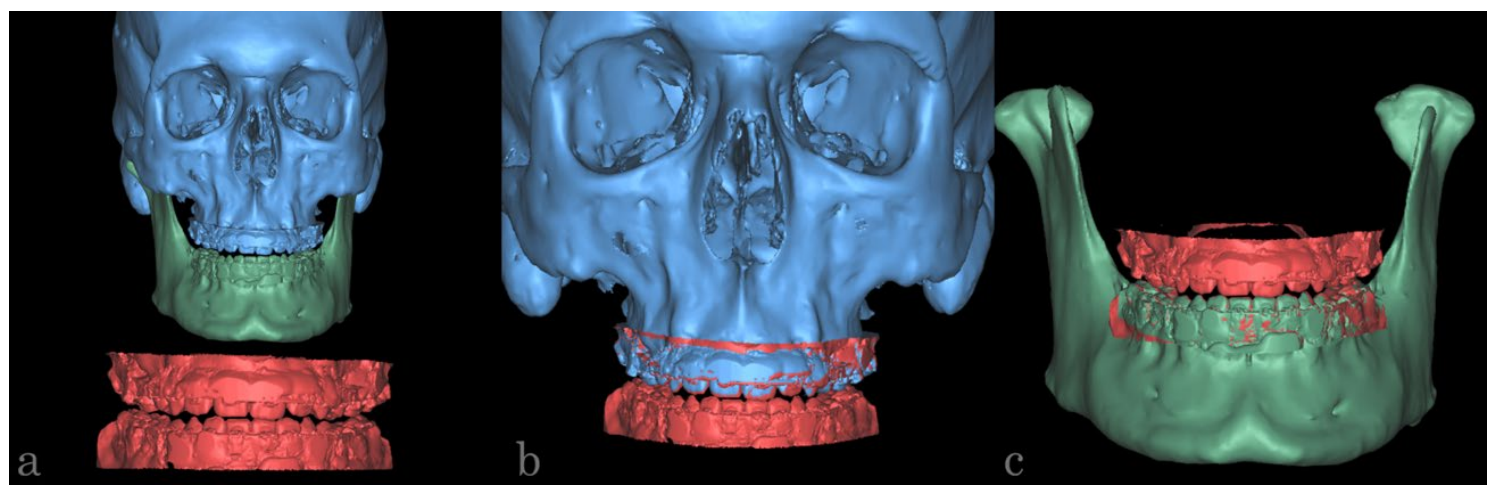

Abbildung 24: Einstellung der Okklusion mithilfe der gescannten präoperativen Zentrikposition. 


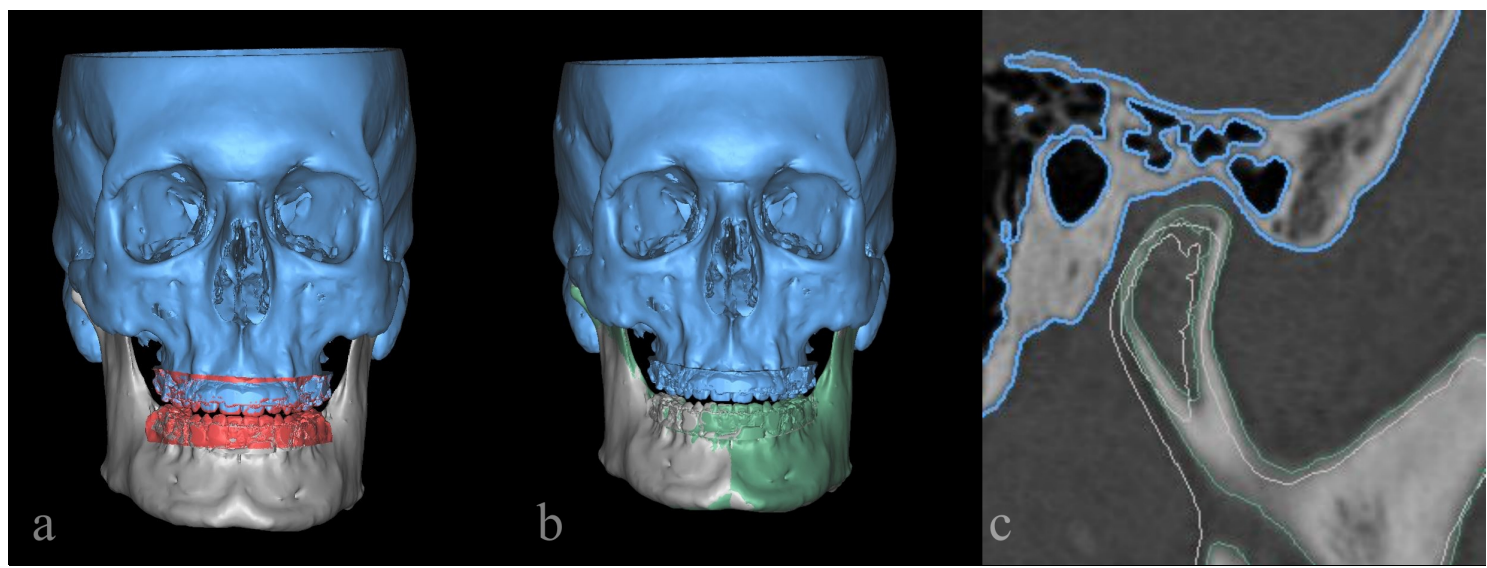

Abbildung 25: a) Herstellung des intraoperativen Unterkiefer Composite-Modelles in grau b) Überlagerung der beiden Unterkieferpositionen: grün präoperative Zentrik, grau intraoperative Zentrik c) sagittaler Schnitt durch einen Kondylus mit beiden Modellen.

Um die vom DICOM-Satz der DVT abweichende Kondylenposition des intraoperativen Registrates in eine zweite Mandibula zu übertragen, wurde erneut die Funktion „Occlusion Registration“ genutzt. Nach dem gleichen Vorgehen wurde auch hier das UnterkieferComposite-Modell inklusive Kondylen, in die durch das digitalisierte intraoperative Registrat bestimmte Position verschoben. Die okklusale Registration erfolgte anhand korrespondierender Punkte und Alignment der gesamten Zahnoberfläche. Das intraoperative Unterkiefer-Composite-Modell wurde als separates Modell gespeichert (Abbildung 25 in grau). Anhand der beiden hergestellen Unterkiefer Composite-Modelle wurde die Datenanalyse durchgeführt.

\subsubsection{Datenanalyse und Auswertung}

$\mathrm{Da}$ als postoperative Veränderungen der Kondylus-Fossa-Relation insbesondere das Absinken des Kondylus nach inferior beschrieben wurde (Reyneke und Ferretti 2002; Ueki et al. 2007), stellte die Breite des superioren Gelenksspalts die Hauptzielgröße der Untersuchung dar. Zudem wurden die Breite des anterioren und posterioren Gelenkspalts, dentale Messwerte zur Untersuchung der okklusalen Verhältnisse, sowie die prozentuale Übereinstimmung der 3D Rekonstruktion des Kondylus prä- und intraoperativ analysiert.

\subsubsection{Cephalometrische Analyse}

Die cephalometrische Analyse fand innerhalb der Software ProPlan ${ }^{\circledR}$ CMF 3.0 statt. Hierzu wurden neben klassischen FRS-Werten, wie SNA, ANB, WITS zur Klassifizierung der Patienten (Tabelle 2), die Kondylen gemäß der validierten Methode nach Pullinger et al. (1986) hinsichtlich des kürzesten Abstandes zwischen den prä- und intraoperativen 
Unterkiefermodellen im Bereich des superioren, anterioren und posterioren Gelenkspaltes vermessen.

Tabelle 2: Klassische FRS-Werte

\begin{tabular}{lll}
\hline SNA & SNA-Winkel & $\begin{array}{l}\text { Winkel zwischen der S-N-Linie } \\
\text { (vordere Schädelbasis) und der N- } \\
\text { A-Linie }\end{array}$ \\
ANB & ANB-Winkel & $\begin{array}{l}\text { Winkel zwischen den Linien N-A } \\
\text { und N-B }\end{array}$ \\
WITS & WITS-Appraisal & $\begin{array}{l}\text { Abstand zwischen A und B Punkt } \\
\text { senkrecht projiziert auf die } \\
\text { Okklusionsebene }\end{array}$ \\
\hline
\end{tabular}

Zur exakten Vermessung der dreidimensionalen Schädelmodelle wurden je Proband 42 Orientierungspunkte gesetzt, davon 19 im Bereich des Oberkiefers und Cranium sowie 23 Punkte jeweils auf das präoperative und intraoperative Unterkiefermodell (Tabelle 3).

Die Definition der zusetzenden Punkte im Bereich des Kondylus und der Fossa orientieren sich an den Studien von Yoon et al. (2015), Chen et al. (2013) und Zafar et al. (2014). Angelehnt an die Arbeit von Gateno et al. (2011) wurden zusätzlich Punkte an den korrespondierenden Molaren gesetzt (Tabelle 4).

Tabelle 3: Cephalometrische Orientierungspunkte für die Vermessung der Kondylenposition prä- und intraoperativ

\begin{tabular}{|c|c|c|}
\hline CA-R & A Punkt Condylus rechts & Superiorster Punkt des Condylus rechts \\
\hline CA-R* & $\begin{array}{l}\text { A Punkt Condylus rechts } \\
\text { intraoperativ }\end{array}$ & $\begin{array}{l}\text { Superiorster Punkt des Condylus rechts auf der } \\
\text { intraoperativen UK-Maske }\end{array}$ \\
\hline CA-L & A Punkt Condylus links & Superiorster Punkt des Condylus links \\
\hline CA-L* & $\begin{array}{l}\text { A Punkt Condylus links in- } \\
\text { traoperativ }\end{array}$ & $\begin{array}{l}\text { Superiorster Punkt des Condylus links auf der } \\
\text { intraoperativen UK-Maske }\end{array}$ \\
\hline CB-R & B Punkt Condylus rechts & Anteriorster Punkt Condylus rechts \\
\hline $\mathrm{CB}-\mathrm{R}^{*}$ & $\begin{array}{l}\text { B Punkt Condylus rechts } \\
\text { intraoperativ }\end{array}$ & $\begin{array}{l}\text { Anteriorster Punkt Condylus rechts auf der } \\
\text { intraoperativen UK-Maske }\end{array}$ \\
\hline CB-L & B Punkt Condylus links & Anteriorster, mittiger Punkt Condylus links \\
\hline CB-L* & $\begin{array}{l}\text { B Punkt Condylus } \\
\text { intraoperativ }\end{array}$ & $\begin{array}{l}\text { Anteriorster Punkt Condylus links auf der } \\
\text { intraoperativen UK-Maske }\end{array}$ \\
\hline CC-R & C Punkt Condylus rechts & Posteriorster, mittiger Punkt Condylus rechts \\
\hline
\end{tabular}




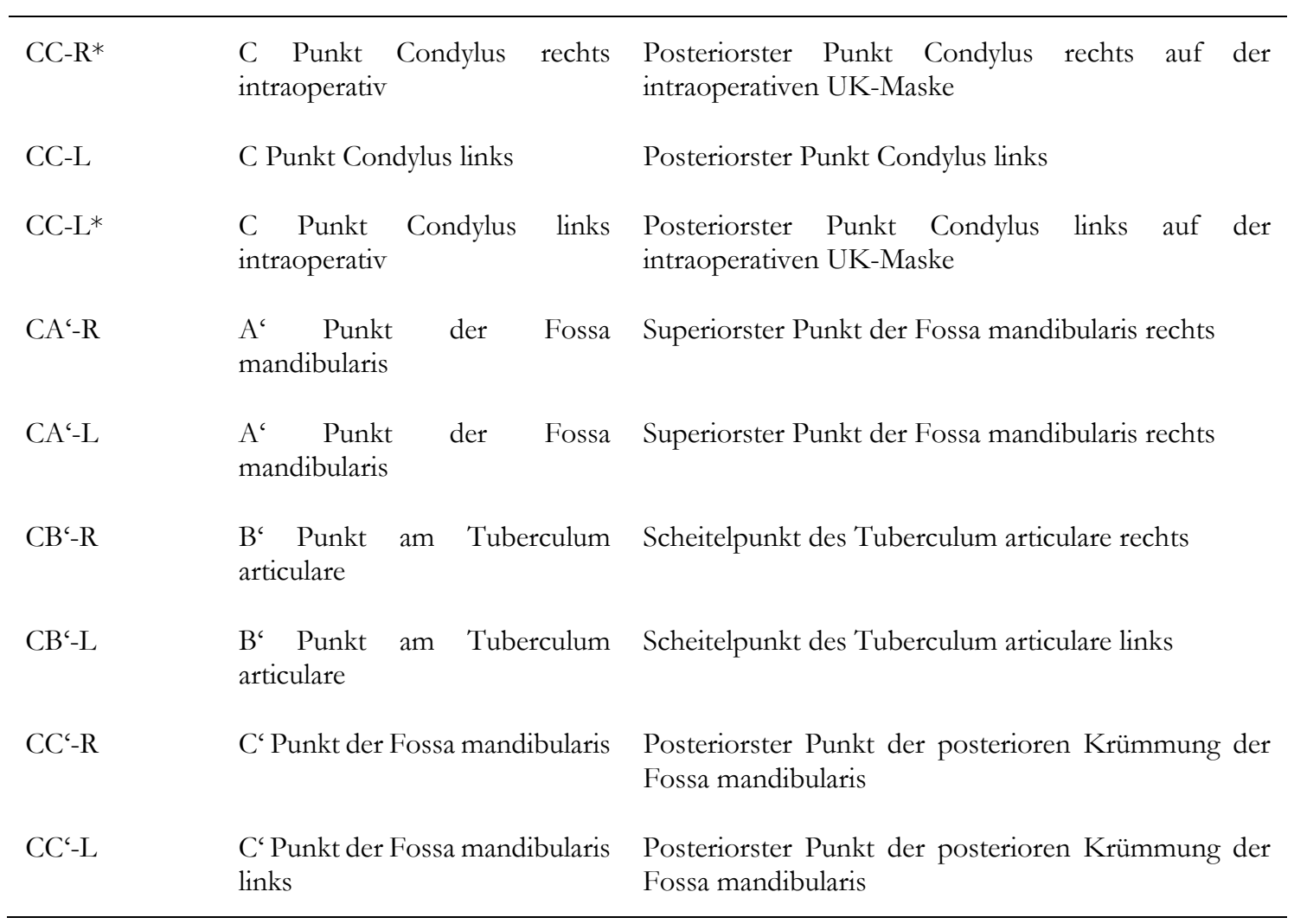

Tabelle 4: Cephalometrische Orientierungspunkte zur Vermessung der dentalen Verhältnisse der prä- und intraoperativen Situation

\begin{tabular}{|c|c|c|}
\hline UR6 & Upper right 6 & Mesiobukkale Höckerspitze 16 \\
\hline UR6* & Upper right 6 intraoperativ & $\begin{array}{l}\text { Mesiobukkale Höckerpitze } 16 \text { auf der intraoperativen } \\
\text { UK-Maske }\end{array}$ \\
\hline UL6 & Upper left 6 & Mesiobukkale Höckerspitze 26 \\
\hline UL6* & Upper left 6 intraoperativ & $\begin{array}{l}\text { Mesiobukkale Höckerspitze } 26 \text { auf der intraoperativen } \\
\text { UK-Maske }\end{array}$ \\
\hline LR6 & Lower right 6 & Bukkale Fissur 46 \\
\hline LR6* & Lower right 6 intraoperativ & Bukkale Fissur 46 auf der intraoperativen UK-Maske \\
\hline LL6 & Lower left 6 & Bukkale Fissur 36 \\
\hline LL6* & Lower left 6 intraoperativ & Bukkale Fissur 36 auf der intraoperativen UK-Maske \\
\hline Ii-Approx & $\begin{array}{l}\text { Approximalkontakt Inzisivi } \\
\text { inferior }\end{array}$ & $\begin{array}{l}\text { Approximalkontakt zwischen den unteren mittleren } \\
\text { Inzisivi }\end{array}$ \\
\hline
\end{tabular}




\begin{tabular}{|c|c|c|c|}
\hline Ii-Approx* & $\begin{array}{l}\text { Approximalkontakt } \\
\text { inferior intraoperativ }\end{array}$ & Inzisivi & $\begin{array}{l}\text { Approximalkontakt zwischen den unteren mittleren } \\
\text { Inzisivi auf der intraoperativen Maske }\end{array}$ \\
\hline
\end{tabular}

Anhand der Orientierungspunkte wurden dreidimensionale Ebenen konstruiert, die als Basis für Winkel- und Streckenmessungen dienten (Tabelle 5, Abbildung 26). Es wurden im Rahmen der cephalometrischen Analyse dreidimensionale Messungen durchgeführt.

Bedingt durch den Algorithmus der Software werden dreidimensionale Messungen immer als kürzeste Strecke zwischen den jeweiligen Punkten berechnet.

Tabelle 5: Konstruierte Ebenen zur cephalometrischen Analyse

\begin{tabular}{lll}
\hline FH & Frankfurter Horizontale & $\begin{array}{l}\text { Porion links und rechts und } \\
\text { Orbita rechts }\end{array}$ \\
UKE & Unterkieferebene & $\begin{array}{l}\text { Durch Menton und caudaler } \\
\text { Ramus links und rechts }\end{array}$ \\
SAG & Unterkieferebene intraoperativ & $\begin{array}{l}\text { Durch Menton* und caudaler } \\
\text { Ramus links und rechts auf der } \\
\text { intraoperativen UK-Maske }\end{array}$ \\
& Sagittalebene & $\begin{array}{l}\text { Senkrecht zu FH und } \\
\text { Frontalebene, durch Punkt N }\end{array}$ \\
\hline
\end{tabular}

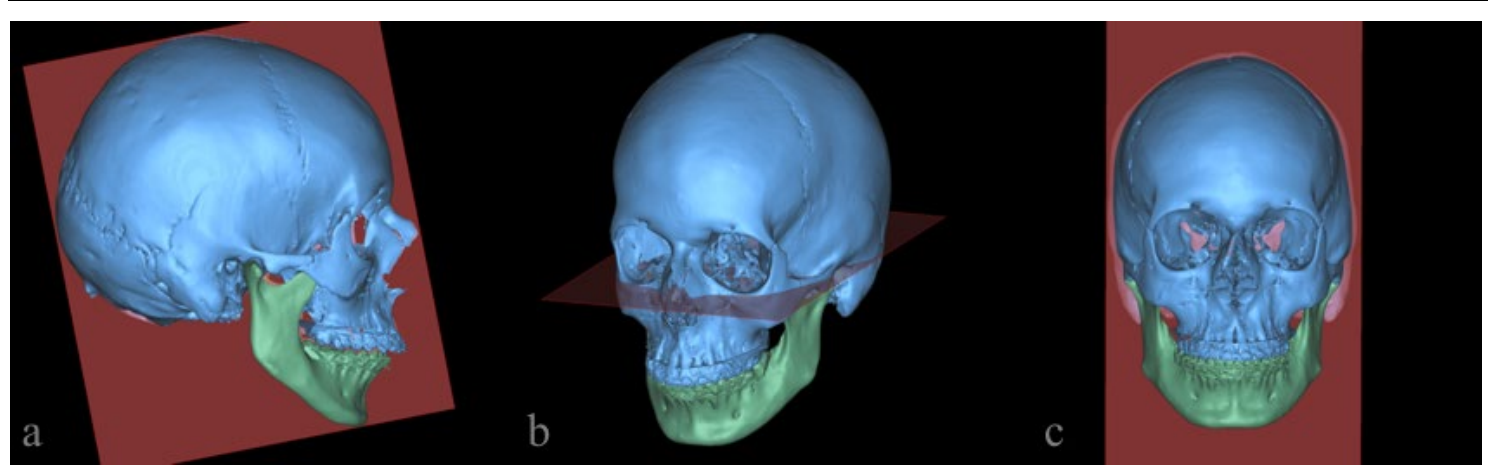

Abbildung 26: Konstruierte Ebenen a) Sagittalebene, b) Frankfurter Horizontale, c) Frontalebene.

Für die Messung der zweidimensionalen Werte, wurden die korrespondierenden Punkte zunächst auf eine Ebene (z. B. Sagittal- oder Frontalebene) gespiegelt und die Abstände anschließend berechnet. Besondere Beachtung fand die cephalometrische Analyse der Gelenkspalte. Diese wurde mit Messungen des anterioren Gelenkspaltes (AJS= anterior joint space), zwischen dem anteriorsten Punkt des Kondylus und dem anteriorsten Punkt der Fossa, dem superioren Gelenkspalt (SJS= superior joint space), zwischen dem superiorsten Punkt des Kondylus und der Fossa und dem posterioren Gelenkspalt (PJS= posterior joint space), zwischen dem posteriorsten Punkt des Kondylus und der Fossa, durchgeführt (Abbildung 27, Tabelle 6). Diese Messungen wurden separat für die präoperative 
Kondylenposition und die intraoperative Kondylenposition (mit * markierten Werte und Messungen), jeweils für den rechten und linken Kondylus erhoben. Zur weiteren Analyse der Kondylenpositionen wurden die Abstände der Orientierungspunkte der Kondylen zu Ebenen, die auf Basis der unbeweglichen Orientierungspunkte der Maxilla/Cranium-Maske konstruiert wurden, gemessen. So wurde unter anderem der Abstand zwischen dem superiorsten Punkt des Kondylus zur Frankfurter Horizontalen, der Abstand zwischen dem anteriorsten und posteriorsten Kondyluspunkt und der Frontalebene, der Abstand zwischen dem medialsten und lateralsten Kondyluspunkt zur Sagittalebene, auf der linken und rechten Seite jeweils prä- und intraoperativ gemessen. Auf dentaler Ebene wurde die sagittale Abweichung des Unterkiefers im Bezug zur Sagittalebene und die Abstände zwischen den korrespondieren ersten Molaren gemessen.

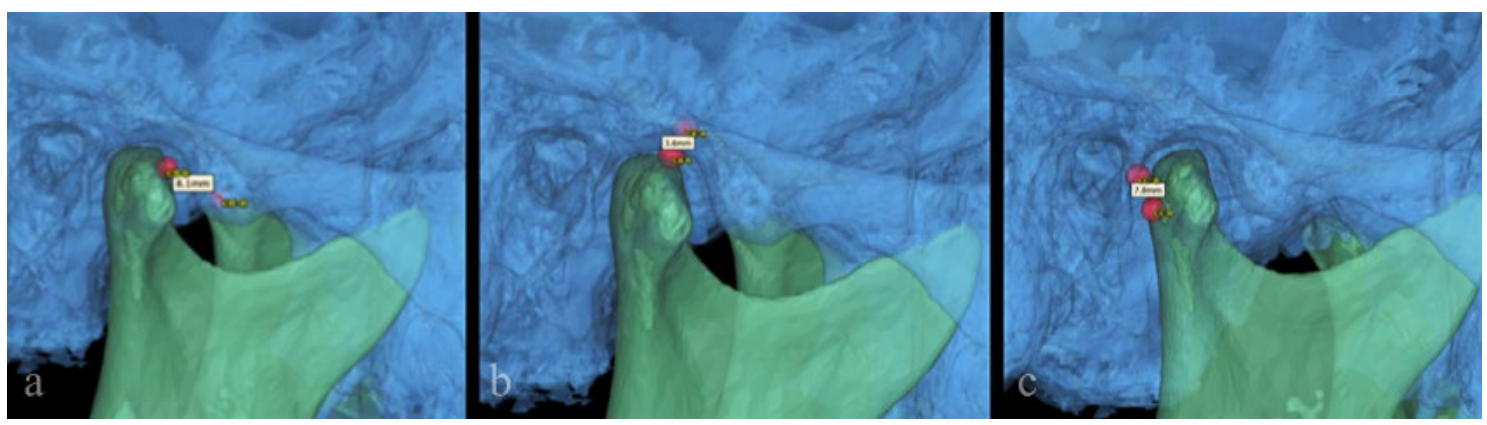

Abbildung 27: Cephalometrische Messungen des a) anterioren, b) superioren und c) posterioren Gelenkspaltes.

Tabelle 6: Cephalometrische Messungen des Gelenkspaltes prä- und intraoperativ und der dentalen Referenzpunkte

\begin{tabular}{|c|c|c|}
\hline $\begin{array}{l}\text { AJS-R } \\
\text { AJS-L }\end{array}$ & $\begin{array}{l}\text { Anteriorer Gelenkspalt (anterior } \\
\text { joint space) }\end{array}$ & $\begin{array}{l}\text { Abstand zwischen dem } \\
\text { anteriorsten Punkt des Kondylus } \\
\text { und dem anteriorsten Punkt der } \\
\text { Fossa rechts und links }\end{array}$ \\
\hline $\begin{array}{l}\text { AJS-R* } \\
\text { AJS-L* }\end{array}$ & $\begin{array}{l}\text { Anteriorer Gelenkspalt (anterior } \\
\text { joint space) intraoperativ }\end{array}$ & $\begin{array}{l}\text { Abstand zwischen dem } \\
\text { anteriorsten Punkt des Kondylus } \\
\text { und dem anteriorsten Punkt der } \\
\text { Fossa rechts und links auf der } \\
\text { intraoperativen UK-Maske }\end{array}$ \\
\hline $\begin{array}{l}\text { SJS-R } \\
\text { SJS-L }\end{array}$ & $\begin{array}{l}\text { Superiorer Gelenkspalt (Superior } \\
\text { joint space) }\end{array}$ & $\begin{array}{l}\text { Abstand zwischen dem } \\
\text { superiorsten Punkt des Kondylus } \\
\text { und dem superiorsten Punkt der } \\
\text { Fossa rechts und links }\end{array}$ \\
\hline $\begin{array}{l}\text { SJS-R* } \\
\text { SJS-L* }\end{array}$ & $\begin{array}{l}\text { Superiorer Gelenkspalt (Superior } \\
\text { joint space) intraoperativ }\end{array}$ & $\begin{array}{l}\text { Abstand zwischen dem } \\
\text { superiorsten Punkt des Kondylus } \\
\text { und dem superiorsten Punkt der } \\
\text { Fossa rechts und links auf der } \\
\text { intraoperativen UK-Maske }\end{array}$ \\
\hline $\begin{array}{l}\text { PJS-R } \\
\text { PJS-L }\end{array}$ & $\begin{array}{l}\text { Posteriorer Gelenkspalt } \\
\text { (posterior joint space) }\end{array}$ & $\begin{array}{l}\text { Abstand } \quad \text { zwischen } \\
\text { posteriorsten } \quad \text { Punkt }\end{array}$ \\
\hline
\end{tabular}


Kondylus und dem posteriorsten Punkt der Fossa rechts und links

\begin{tabular}{|c|c|c|}
\hline $\begin{array}{l}\text { PJS-R* } \\
\text { PJS-L* }\end{array}$ & $\begin{array}{lrr}\text { Posteriorer } & \text { Gelenkspalt } \\
\text { (posterior } & \text { joint } & \text { space) } \\
\text { intraoperativ } & & \end{array}$ & $\begin{array}{l}\text { Abstand zwischen dem } \\
\text { posteriorsten Punkt des } \\
\text { Kondylus und dem posteriorsten } \\
\text { Punkt der Fossa rechts und links } \\
\text { auf der intraoperativen UK- } \\
\text { Maske }\end{array}$ \\
\hline $\begin{array}{l}\text { Ii-Approx-SAG } \\
\text { Ii-Approx-SAG* }\end{array}$ & $\begin{array}{l}\text { Deviation der Dentition prä- und } \\
\text { intraoperativ }\end{array}$ & $\begin{array}{l}\text { Abstand zwischen dem } \\
\text { Aproximalkontakt der mittleren } \\
\text { Inzisivi zur Sagittalebene auf der } \\
\text { prä- und intraoperativen UK- } \\
\text { Maske }\end{array}$ \\
\hline $\begin{array}{l}\text { UR6-LR6 } \\
\text { UR6-LR6* }\end{array}$ & $\begin{array}{l}\text { Höcker-Fossa Korrelation rechts } \\
\text { prä-und intraoperativ }\end{array}$ & $\begin{array}{l}\text { Abstand zwischen den } \\
\text { korrespondierenden Molaren } \\
\text { rechtsseitig auf der prä- und } \\
\text { intraoperativen UK-Maske }\end{array}$ \\
\hline $\begin{array}{l}\text { UL6-LL6 } \\
\text { UL6-LL6* }\end{array}$ & $\begin{array}{l}\text { Höcker-Fossa Korrelation links } \\
\text { prä-und intraoperativ }\end{array}$ & $\begin{array}{l}\text { Abstand zwischen den } \\
\text { korrespondierenden Molaren } \\
\text { linksseitig auf der prä- und } \\
\text { intraoperativen UK-Maske }\end{array}$ \\
\hline
\end{tabular}

\subsubsection{Analyse der Volumina}

Ergänzend zur cephalometrischen Auswertung wurde eine Überlagerung der Gelenkpositionen durchgeführt (Abbildung 28a). Hierfür wurden die Kondylen in ProPlan ( CMF 3.0 vom Mandibulacorpus getrennt. Die Schnittebene wurde parallel zur Frankfurter Horizontalen durch die Inzisura mandibulae separat links und rechts konstruiert. Alle Kondylenpositionen wurden als $s t$-Datei aus ProPlan ${ }^{\circledR}$ exportiert und in die Software Mimics Research ${ }^{\circledR}$ (Materialise, Belgien) eingepflegt. In Mimics Research ${ }^{\circledR}$ wurden über die Funktion ,global registration" die korrespondierenden Dreiecke der stl-Dateien ermittelt und anschließend eine ,part comparsion analyse" durchgeführt. Hierbei kalkuliert der Algorithmus des tools die geringste Distanz zwischen den vergleichbaren Dreieckspunkten des ausgewählten Objektes (intraoperative Kondylenposition) und dem Referenzobjekt (präoperative Kondylenposition). Berechnet werden so die minimale und maximale Abweichung, sowie der Medianwert, die zur Validierung der Methodik im Rahmen der Interrater-Reliabilität genutzt wurden. Die Ergebnisse stellt das Tool als Heat-Map bereit (Abbildung 28 b). 


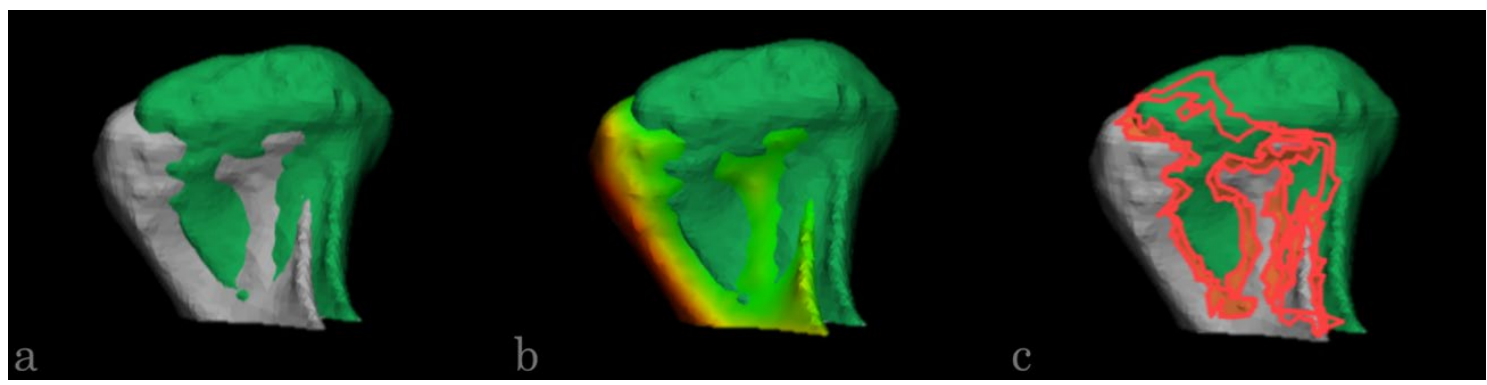

Abbildung 28: a) überlagerte Kondylenpositionen b) Heat-Map der Kondylenpositionen c) kollidierendes Volumenanteile.

Mithilfe des tools „collision detected" in Mimics Research ${ }^{\circledR}$ wurde eine Analyse des kollidierenden Volumensanteils der Kondylen durchgeführt (Abbildung 28c).

Im tool ,collision detected" wurden einerseits das reine Volumen des präoperativ registrierten Kondylus (Angabe in $\mathrm{mm}^{3}$ ), andererseits das Volumen der deckungsgleichen Bereiche der prä- und intraoperativ registrierten Kondylenpositionen bestimmt (Abbildung 29).

Die prozentuale Volumenübereinstimmung rechts/links berechnete sich als Quotient beider Volumina.

$$
\text { Volumen-R/Volumen-L }=\frac{\begin{array}{c}
\text { Volumen deckungsgleicher Bereich } \\
\text { prä-und intraoperative Kondylenposition }
\end{array}}{\text { Volumen präoperativer Kondylus }}
$$

Diese Untersuchung wurde separat für den rechten und linken Kondylus durchgeführt.

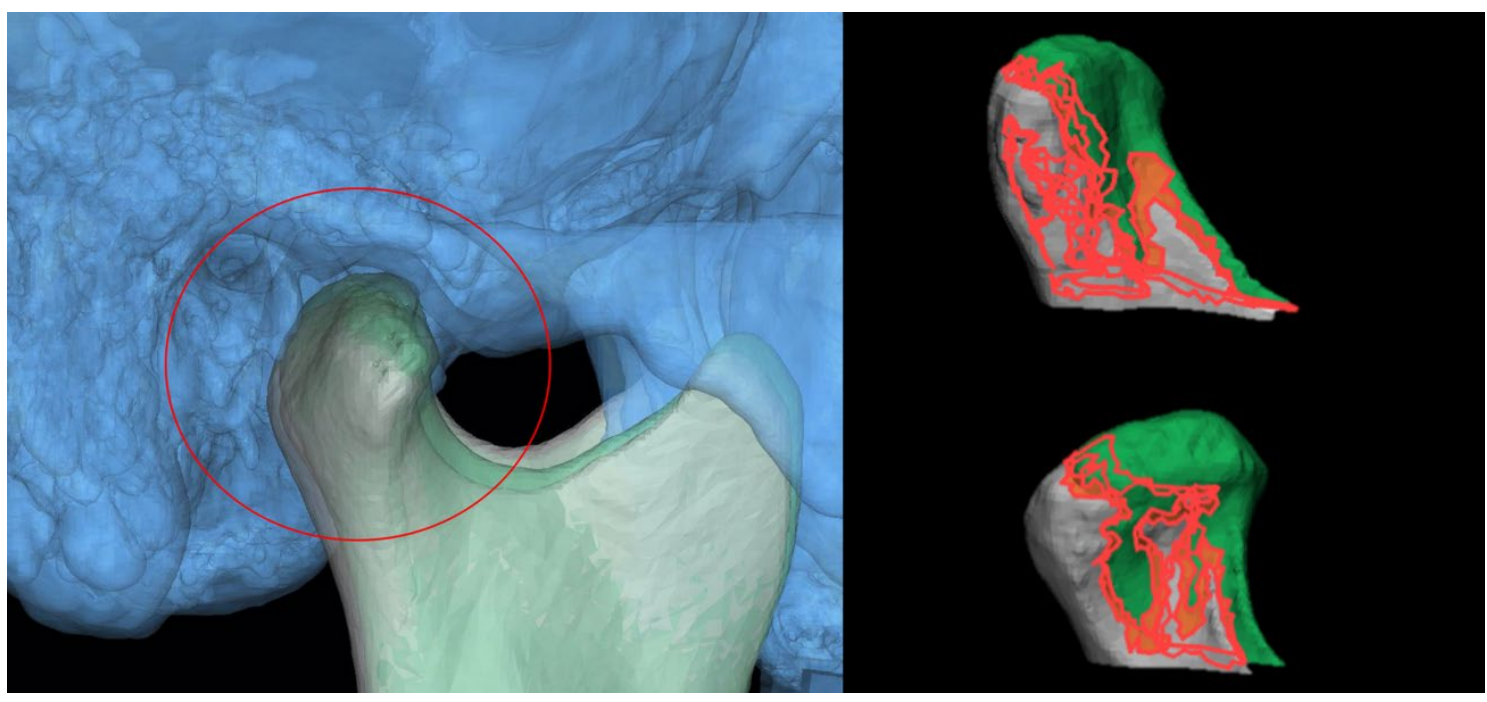

Abbildung 29: Darstellung der deckungsgleichen Bereiche der prä-und intraoperativen Kondylenpositionen.

\subsubsection{Interrater-Reliabilität}

Zur Validierung der Methodik zwischen unterschiedlichen Untersuchern wurde die Interrater-Reliabilität bei 10 Patienten des Kollektivs durchgeführt. Die zweite Untersucherin 
war kundig in der Software Mimics ${ }^{\circledR}$ InPrint 3.0 und ProPlan ${ }^{\circledR}$ CMF 3.0, sodass Anwendungsfehler ausgeschlossen werden können.

Zunächst wurde die Genauigkeit der Rekonstruktion der Kondylen aus dem dreidimensionalen Datensatz des DVTs überprüft. Hierzu wurden in Mimics ${ }^{\circledR}$ InPrint 3.0, nach selbiger Vorgehensweise wie oben erläutert von beiden Untersuchern zu einem zweiten Zeitpunkt die Kondylen rekonstruiert, nachgearbeitet und geglättet. Zur Analyse wurden die Kondylen in ProPlan ${ }^{\circledR}$ CMF 3.0 vom Mandibulacorpus parallel zur Frankfurter Horizontalen durch die Inzisura mandibulae getrennt und über Mimics Research ${ }^{\circledR}$ ausgewertet.

Des Weiteren fand eine Analyse in Bezug auf die cephalometrischen Werte statt. Dieselbe cephalometrische Analyse wie oben vorgestellt, wurde von der zweiten Untersucherin ohne weitere Hilfestellung durchgeführt.

\subsubsection{Intrarater-Reliabilität}

Von Untersucherin eins wurde zu einem zweiten Zeitpunkt die cephalometrische Analyse bei 10 Patienten des Kollektivs wiederholt.

\subsection{Statistische Auswertung}

Die statistische Analyse wurde mithilfe des Statistikprogrammes IBM SPSS Statistics für Windows (Version 25, IBM Corp. Armonk, NY, USA) durchgeführt. Als globales Signifikanzniveau wurde $p<0,05$ angenommen.

\subsubsection{Statistische Auswertung der cephalometrischen Messungen}

Pro Patienten wurden 13 cephalometrische Messwerte zum Vergleich der prä- und intraoperativen Kondylen- und Mandibulapositionen ausgewertet. Hierzu wurde der Wilcoxon-Vorzeichen-Rang-Text für gepaarte Stichproben angewandt.

\subsubsection{Statistische Auswertung der volumetrischen Übereinstimmung}

Die erhobenen Daten wurden als nicht normalverteilt angenommen. Zur Analyse der volumetrischen Übereinstimmung wurde der Einstichproben Wilcoxon-Vorzeichen-RangTest verwendet. Die Auswertung fand separat für den linken und rechten Kondylus der Probanden statt. Als theoretischer Wert der Volumenkongruenz wurde 100\% angenommen. Statistisch signifikante $p$-Werte wurden unter Verwendung der Bonferroni-Korrektur berichtigt.

Zudem wurde eine Subgruppenanalyse zum Vergleich von skelettaler Klasse II und III und symmetrischen und asymmetrischen Patienten vorgenommen. Hierzu wurden die volumetrischen Übereinstimmungen der Kondylen links und rechts mithilfe des Mann-Whitney U- 
Test für unabhängige Stichproben analysiert. Im Rahmen dieser Subgruppenanalyse wurde bei den asymmetrischen und symmetrischen Patienten das Volumen der Kondylen zusätzlich in Betrachtung der Deviation- und Nichtdeviationsseite verglichen. Hierzu wurde der Wilcoxon-Vorzeichen-Rang-Test angewandt.

\subsubsection{Interrater-Reliabilität}

Zur Untersuchung der Interrater-Reliabilität zur Genauigkeit der Kondylenrekonstruktion konnten über eine Heat-Map die minimalen sowie maximalen Abweichung und die daraus resultierenden Mediane und Mittelwerte abgeleitet werden und verglichen werden.

Zum Vergleich der cephalometrischen Werte mit Untersucherin eins wurde ein BlandAltman-Plot für alle Messungen angefertigt

\subsubsection{Intrarater-Reliabilität}

Nach selbigem Vorgehen wie bei der Interrater-Reliabilität wurde zum Vergleich der cephalometrischen Werte der Bland-Altman-Plot verwendet. 


\section{Ergebnisse}

Im Rahmen dieser Untersuchung wurden die Kondylenpositionen an zwei unterschiedlichen Zeitpunkten von 30 Patienten mit skelettaler Klasse I, II und III dreidimensional cephalometrisch und dreidimensional volumetrisch analysiert und ausgewertet. Die im folgenden dargestellten Ergebnisse beziehen sich auf das gesamte Patientengut, zusätzlich hierzu auf eine Subgruppenanalyse zwischen Klasse II und Klasse III und einer Subgruppenanalyse zwischen asymmetrisch und symmetrischen Patienten. Die Untersuchungsgruppe der Klasse I ist für eine statistische Auswertung zu gering und wurde daher bei der Subgruppenanalyse der skelettalen Klassen vernachlässigt. Von den 30 Probanden wurden insgesamt 60 Zentrikregistrate und 120 Kondylenpositionen ausgewertet.

\subsection{Patientengut}

\subsubsection{Alter des Probandenkollektivs}

Durchschnittlich betrug das Alter der Patienten zum Operationszeitpunkt 25,8 Jahre. Der Schwerpunkt der Altersverteilung lag deutlich im Bereich zwischen 20 und 29 Jahren (Abbildung 30).

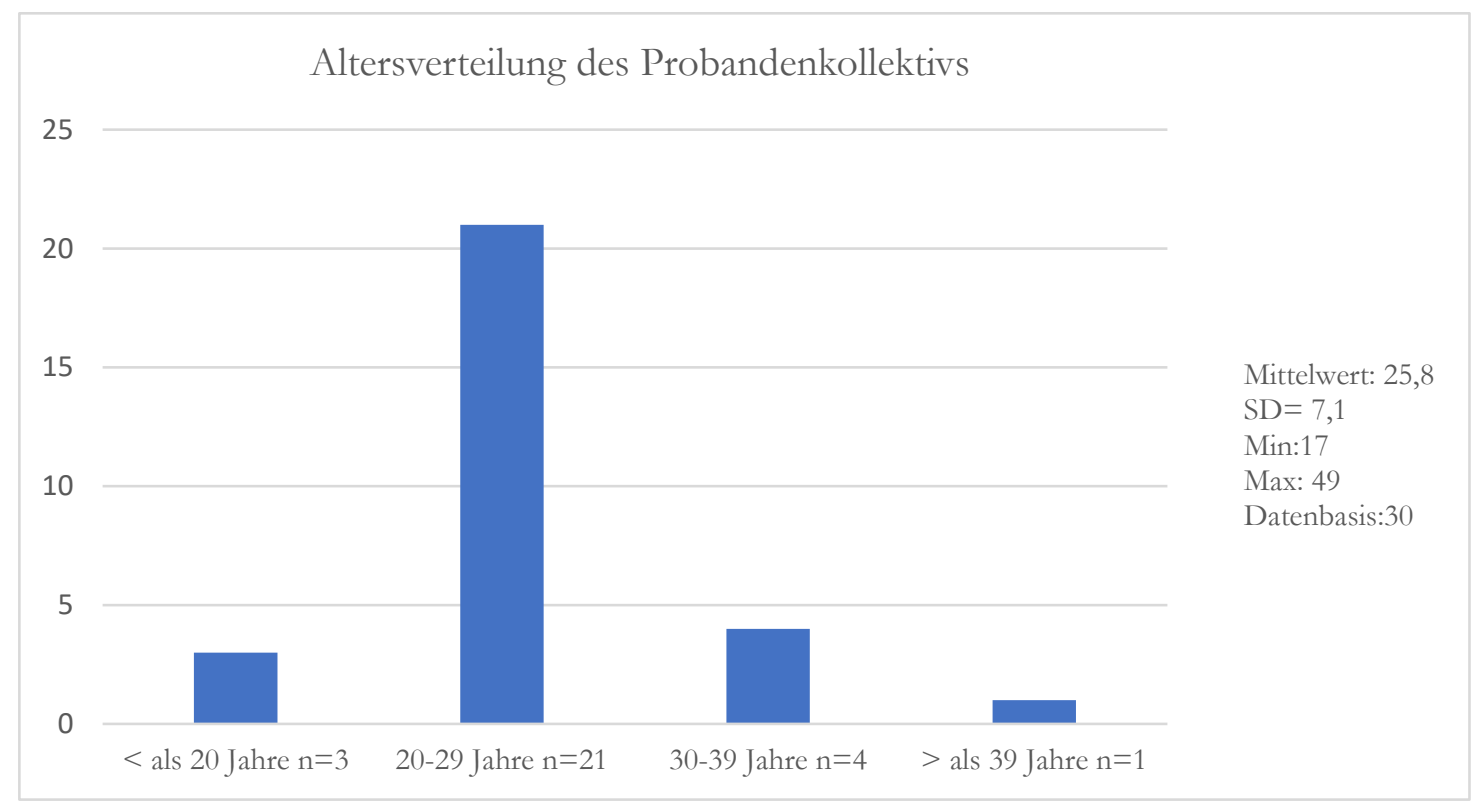

Abbildung 30: Alter des Probandenkollektivs. Aufgezeigt wird auf der Y-Achse die Anzahl der Patienten der entsprechenden Altersgruppe auf der X-Achse.

\subsubsection{Aufteilung des Probandenkollektivs}

In Bezug auf das Geschlecht war das Probandenkollektiv ausgeglichen und bestand aus 14 männlichen und 16 weiblichen Probanden. Die Aufteilung zwischen den skelettalen 
Gruppen II und III war mit je $n=13$ und n=14 ebenfalls ausgeglichen. Lediglich die skelettale Klasse I war mit n=3 unterrepräsentiert. Die Unterteilung des Probandenkollektiv bzgl. der Symmetrie zeigte eine doppelt so hohe Anzahl an symmetrischen Patienten $(n=20)$ als asymmetrische Patienten $(\mathrm{n}=10)$.

22 der 30 Patienten bekamen eine bimaxilläre Operation, d.h. eine Kombination aus einer bilateralen sagittalen Split Osteotomie (BSSO) und einer Le Fort 1 Operation. Sechs der Patienten wurden mit einer isolierten BSSO chirurgisch therapiert, zwei Patienten ausschließlich mit einer Le Fort 1 Operation. Durch die stark asymmetrische Verteilung dieser Gruppen wurde auf eine weitere Analyse diesbezüglich verzichtet.

Insgesamt schien das Probandenkollektiv eine gute Vergleichbarkeit aller relevanten Subgruppen aufzuweisen.

\subsection{Intra-/Interrater-Genauigkeit}

\subsubsection{Interrater-Genauigkeit}

Über die Heat-Map der Kondylen ließ sich die Übereinstimmung der Kondylen beurteilen (Abbildung 31). Die grün gefärbten Bereiche symbolisierten identische Oberflächendreiecke des stl-Datensatzes, gelb-orange Bereiche eine Abweichung von 0,75-1,25mm, rote Bereiche eine Abweichung von 1,25-1,5mm. Die Abweichung in mm bei den ausgewerteten Kondylen $\mathrm{n}=20$ war so gering, dass eine klinische Relevanz ausgeschlossen werden konnte (Tabelle 7).

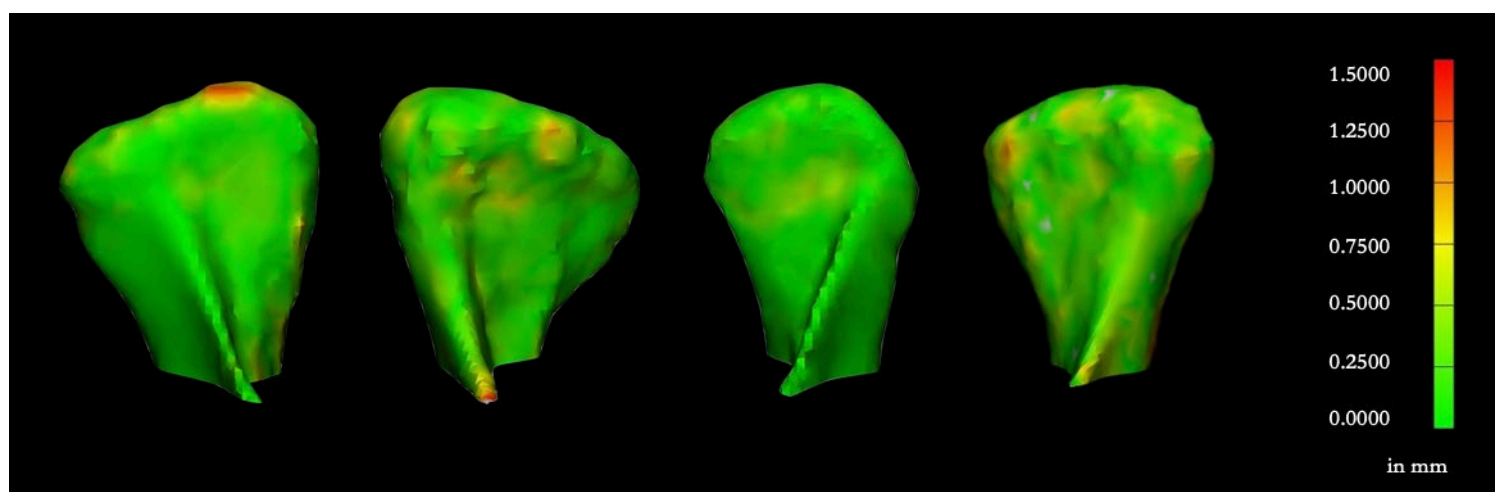

Abbildung 31: Heat-Map der überlagerten Kondylen mit Skala in mm.

In Tabelle 8 sind die relevantesten cephalometrischen Messungen gezeigt, mit dem Mittelwert der Differenz, dem Mittelwert der Differenz plus 1,96* Standardabweichung der Differenz $(\mathrm{LoA}+)$ und dem Mittelwert der Differenz minus 1,96* Standardabweichung der Differenz (LoA-). Beispielhaft zeigen Abbildung 32 und Abbildung 33 den Bland-AltmanPlot für den SJS_L-intraoperativ und den AJS_R-intraoperativ. Alle Punkte lagen innerhalb der limits of agreement. 
Tabelle 7: Ergebnisse der Abweichungen der kondylären Rekonstruktion zwischen den Untersucherinnen

\begin{tabular}{lllll}
\hline Anzahl der Kondylen & Minimum & Maximum & Median & Mittelwert \\
$\mathrm{N}=20$ & 0 & 1,51 & 0,11 & 0,18 \\
\hline
\end{tabular}

Tabelle 8: Tabellarische Darstellung des Bland-Altman-Plots

\begin{tabular}{|c|c|c|c|c|c|c|}
\hline \multirow[t]{2}{*}{ Messwerte in $\mathrm{mm}$} & \multicolumn{4}{|c|}{ Präoperative CR } & \multicolumn{2}{|c|}{ Intraoperative CR } \\
\hline & mDiff & $\mathrm{LoA}+$ & LoA- & mDiff & $\mathrm{LoA}+$ & LoA- \\
\hline Ii-Approx_SAG (mm) & $-0,23$ & 0,9 & $-1,36$ & $-0,94$ & 3,47 & $-5,35$ \\
\hline AJS-R & $-0,86$ & 5,41 & $-7,13$ & $-1,4$ & 2,09 & $-1,47$ \\
\hline AJS-L & $-3,04$ & 2,29 & $-8,37$ & $-2,66$ & 1,76 & $-7,08$ \\
\hline SJS-R & 1,04 & 2,9 & $-0,822$ & 1,38 & 4,81 & $-2,05$ \\
\hline SJS-L & 0,61 & 2,01 & $-0,795$ & 0,76 & 2,01 & $-0,49$ \\
\hline PJS-R & 1,3 & 8,08 & $-5,48$ & 1,4 & 4,92 & $-2,12$ \\
\hline PJS-L & $-0,39$ & 2,33 & $-3,11$ & $-0,15$ & 2,28 & $-2,58$ \\
\hline
\end{tabular}

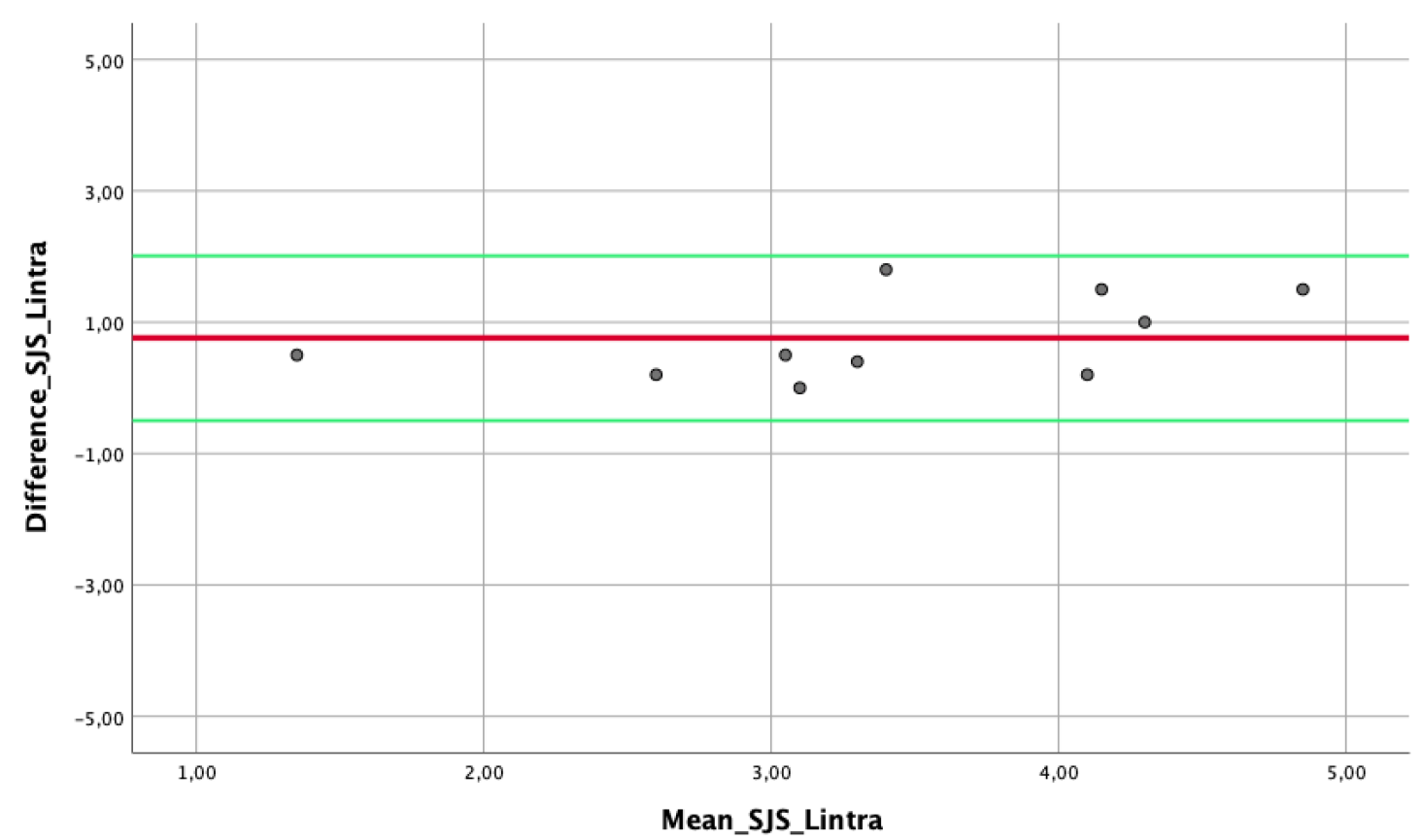

Abbildung 32: Bland-Altman-Plot des SJS links intraoperativ. Auf der Y-Achse wird die Differenz zweier Messungen aufgezeigt, auf der X-Achse finden sich der Mittelwert zweier Messungen. Zur leichteren 
Interpretation beschreibt die rote Linie den Mittelwert aller Messungen während die grünen Linien LoA+ und LoA- darstellen.

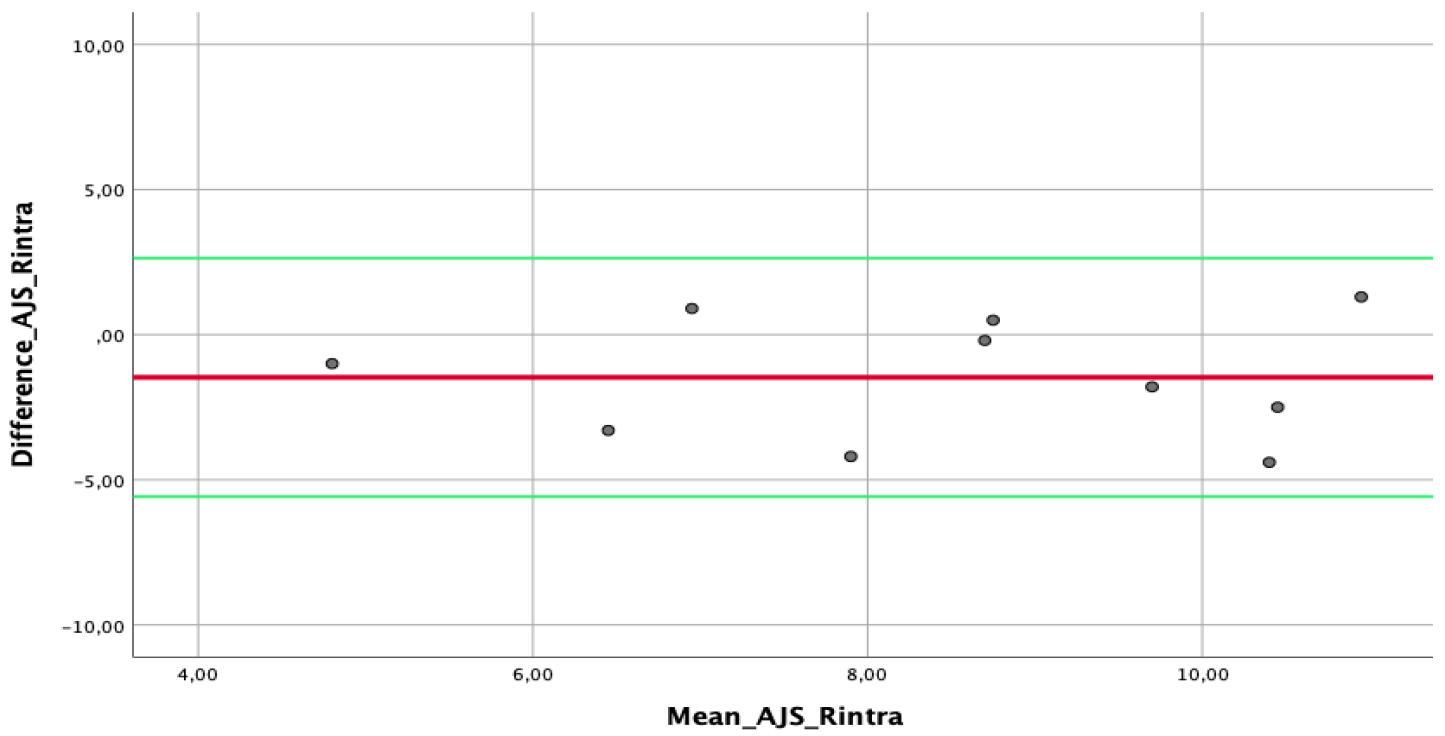

Abbildung 33: Bland-Altman-Plot des AJS rechts intraoperativ. Auf der Y-Achse wird die Differenz zweier Messungen aufgezeigt, auf der X-Achse finden sich der Mittelwert zweier Messungen. Zur leichteren Interpretation beschreibt die rote Linie den Mittelwert aller Messungen während die grünen Linien LoA+ und LoA- darstellen.

\subsubsection{Intrarater-Genauigkeit}

Von Untersucherin eins wurde zu einem zweiten Zeitpunkt die cephalometrische Analyse bei 10 Patienten des Kollektivs wiederholt. Nach selbigem Vorgehen wie bei der InterraterReliabilität wurde zum Vergleich der Ergebnisse der Bland-Altman-Plot verwendet (Tabelle 9). Exemplarisch wird der Bland-Altman-Plot von den Messungen ANB und SJS_L (Abbildung 34 und Abbildung 35) gezeigt. Alle Punkte lagen innerhalb der limits of agreement. Das Toleranzintervall variierte jedoch stark zwischen den Messungen. Während der Messwert für die dentale sagittale Abweichung präoperativ ein Intervall von 2,1 $\mathrm{mm}$ umfasste, lag es für den anterioren Gelenkspalt der rechten Seite präoperativ bei 7,4mm.

Tabelle 9: Tabellarische Darstellung des Bland-Altman

\begin{tabular}{|c|c|c|c|c|c|c|}
\hline \multirow[t]{2}{*}{ Messwerte in $\mathrm{mm}$} & \multicolumn{3}{|c|}{ Präoperative CR } & \multicolumn{3}{|c|}{ Intraoperative CR } \\
\hline & mDiff & $\mathrm{LoA}+$ & LoA- & mDiff & $\mathrm{LoA}+$ & LoA- \\
\hline Ii-Approx_SAG (mm) & $-0,2$ & 0,852 & $-1,25$ & $-0,11$ & 0,811 & $-1,03$ \\
\hline AJS-R & 0,85 & 4,55 & $-2,85$ & 0,09 & 2,69 & $-2,87$ \\
\hline AJS-L & 0,39 & 3,33 & $-2,55$ & 0,28 & 1,73 & $-1,17$ \\
\hline SJS-R & 0,54 & 1,85 & $-0,77$ & 0,55 & 2,52 & $-1,42$ \\
\hline SJS-L & 0,22 & 1,64 & $-1,2$ & $-0,01$ & 1,96 & $-1,98$ \\
\hline
\end{tabular}




\begin{tabular}{lcccccc}
\hline PJS-R & $-0,08$ & 2,47 & $-2,31$ & 0,13 & 0,81 & 1,71 \\
PJS-L & 0,05 & 1,46 & $-2,81$ & 0,14 & 2,62 & $-2,34$ \\
\hline
\end{tabular}

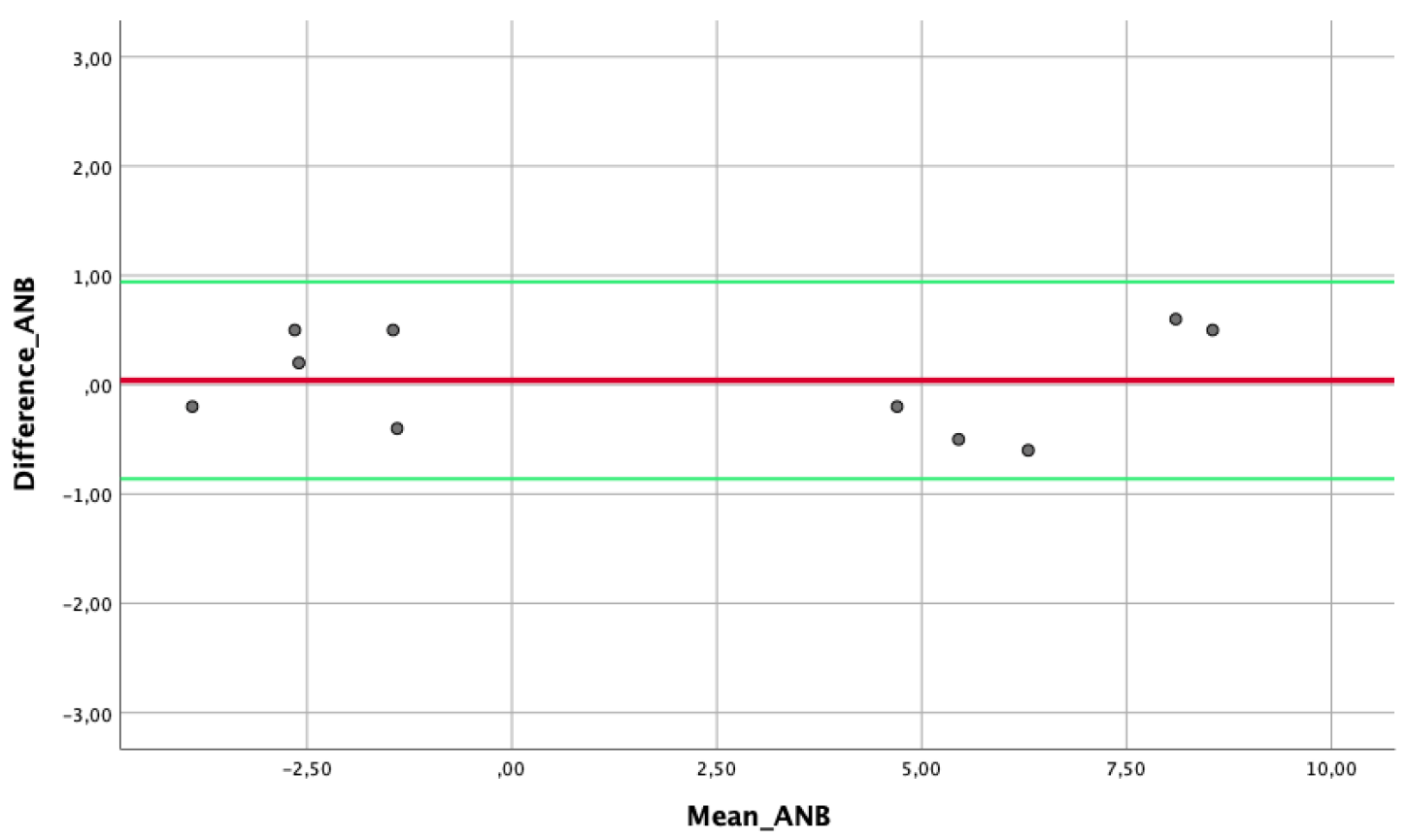

Abbildung 34: Bland-Altman-Plot des ANB. Auf der Y-Achse wird die Differenz zweier Messungen aufgezeigt, auf der X-Achse finden sich der Mittelwert zweier Messungen. Zur leichteren Interpretation beschreibt die rote Linie den Mittelwert aller Messungen während die grünen Linien LoA+ und LoA- darstellen. 


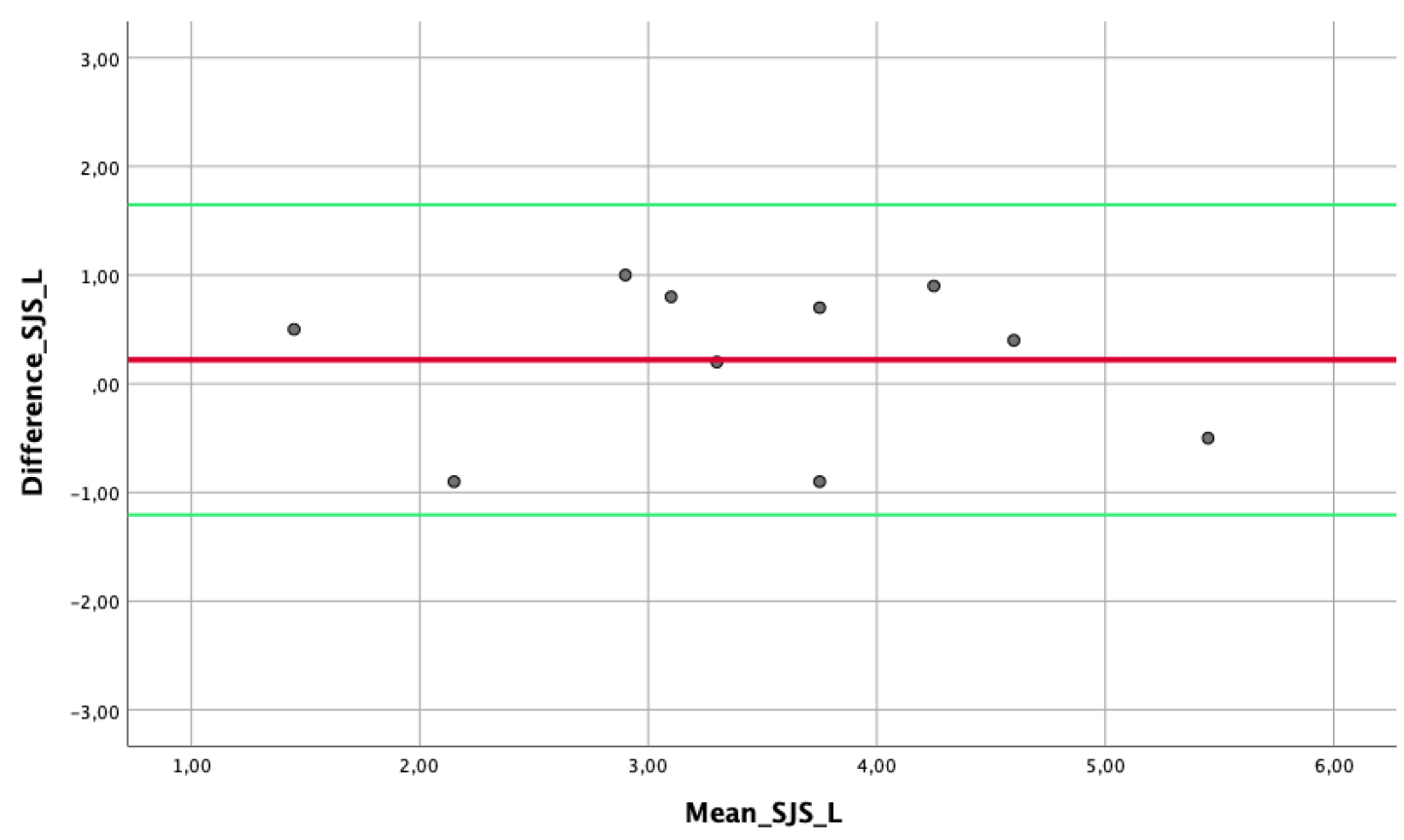

Abbildung 35: Bland-Altman-Plot des SJS links präoperativ. Auf der Y-Achse wird die Differenz zweier Messungen aufgezeigt, auf der X-Achse finden sich der Mittelwert zweier Messungen. Zur leichteren Interpretation beschreibt die rote Linie den Mittelwert aller Messungen während die grünen Linien LoA+ und LoA- darstellen.

\subsection{Analyse der Registratdicke}

Aufgrund unterschiedlicher vertikaler Konfigurationen der Probanden zeigten die mesialen Messpunkte eine höhere Streuung der Registratdicke als die Distalen (Tabelle 10). Da die Abweichung der Mittelwerte zwischen prä-und intraoperativen Messpunkten geringer als $0,05 \mathrm{~mm}$ war, ließ sich ein klinisch relevanter vertikaler Fehler ausschließen.

Tabelle 10: Dicke der Zentrikregistrate in $\mathrm{mm}$

\section{Präoperatives Registrat}

$\mathrm{M}$

$\mathrm{SD}$
16

13

$0,46 \quad 0,97$

$0,07 \quad 1,70$

23

1,04

0,78
26

0,40

0,07
Intraoperatives Registrat

16

13

23 26

$\begin{array}{llll}0,46 & 1,01 & 1,02 & 0,45\end{array}$

$\begin{array}{llll}0,28 & 1,34 & 1,70 & 0,00\end{array}$




\subsection{Ergebnisse der cephalometrischen Analyse}

\subsubsection{Ergebnisse der FRS-Werte}

Die linearen cephalometrischen Messungen zeigen keine signifikanten Änderungen zwischen den prä- und intraoperativen Mandibulapositionen (Tabelle 11).

Der SNB-Winkel wies zwischen prä-und intraoperativer Position ebenso geringe Änderungen wie der ANB-Winkel und der ML/NL-Winkel auf, es kommt zu keiner posterioren Rotation der Mandibula durch eine vertikale Bisssperrung.

Sowohl der ANB- als auch der SNB-Winkel beschreiben die anterior-posteriore Lage der Mandibula in Relation zur Maxilla bzw. zur anterioren Schädelbasis. Dennoch kann auch hier eine posteriore Rotation der Mandibula zu einer Vergrößerung der Winkel führen (Segner und Hasund 1998). Ebenso wie beim ML/NL-Winkel lässt sich jedoch ein vertikaler Fehler ausschließen. Die sagittale Veränderung der Mandibulaposition war bei einer linearen cephalometrischen Messung nicht ausreichend, um determiniert zu werden.

Tabelle 11: cephalometrische Ergebnisse der FRS Werte

\begin{tabular}{lrrr}
\hline & Präoperative CR, median (min- & Intraoperative CR, median (min- & max) \\
Messwert & $76,8(70,1-89,8)$ & $76,9(70,2-89,7)$ & 0,769 \\
SNB $\left(^{\circ}\right)$ & $2,7(-7,9-11,6)$ & $3(-7,9-12,1)$ & 0,739 \\
ANB $\left(^{\circ}\right)$ & $29,2(12,7-43,9)$ & $29,3(12,6-43,3)$ & 0,259 \\
ML/NL $\left(^{\circ}\right)$ & & & \\
\hline
\end{tabular}

\subsubsection{Ergebnisse der transversalen und dentalen cephalometrischen Messungen}

Neben den klassischen FRS-Werten wurden auch dentale lineare Messungen durchgeführt (Tabelle 12).

Die transversale Mittellinienabweichung auf dentaler Ebene zeigte keine signifikanten Veränderungen zwischen der prä-und intraoperativen Mandibulaposition. Im Mittel betrug die Abweichung vom interinzisalen Approximalraum zur Median-Sagittalebene 0,6mm präoperativ und 0,5mm intraoperativ (Abbildung 36).

Auch die linearen dentalen Messpunkte im Bereich der korrespondieren Molaren zeigten weder im 1./4. Quadranten, noch im 2./3. Quadranten signifikante Änderungen der Position.

Tabelle 12: Ergebnisse der transversalen und dentalen cephalometrischen Messungen 


\begin{tabular}{lrrr}
\hline Messwert & Präoperative CR, median (min- & Intraoperative CR, median (min- & max) \\
Ii-Approx-SAG $(\mathrm{mm})$ & $0,6(-6,9-6,8)$ & $0,5(-7,1-6,8)$ & 0,77 \\
UR6-LR6 $(\mathrm{mm})$ & $5,1(1,8-9,6)$ & $5,2(1,9-9,2)$ & 0,206 \\
UL6-LL6 $(\mathrm{mm})$ & $4,9(1,7-8,9)$ & $5,1(1,6-9,1)$ & 0,461 \\
\hline
\end{tabular}

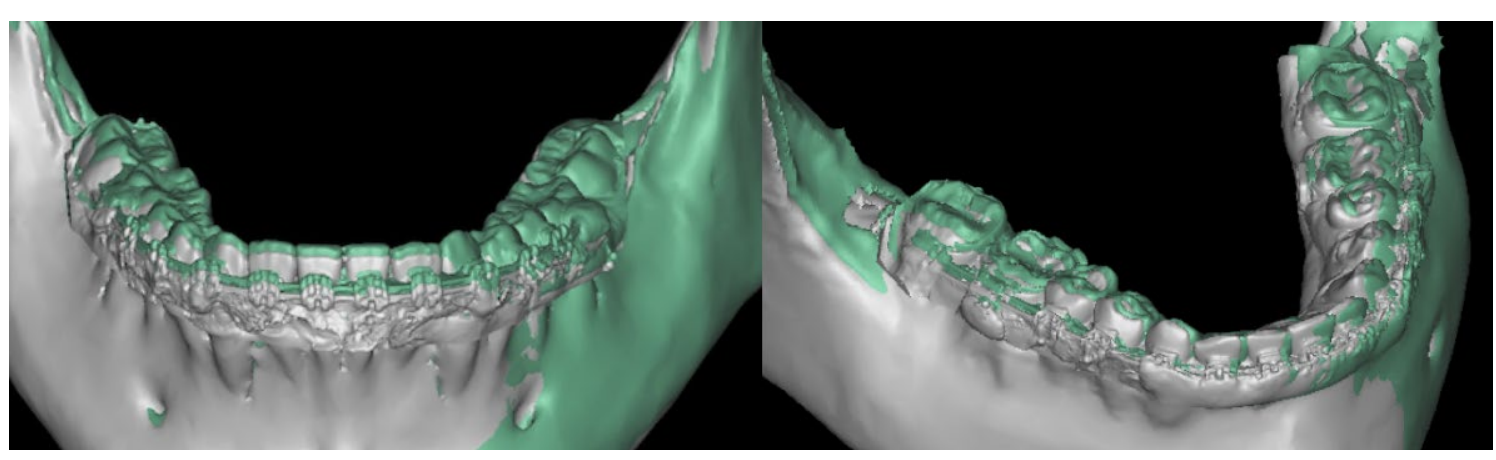

Abbildung 36: Darstellung der dentalen Abweichungen zwischen präoperativer (grün) und intraoperativer (grau) Zentrik.

\subsubsection{Ergebnisse der cephalometrischen Gelenkspaltmessungen}

Zur linearen Vermessung des Gelenkspaltes wurden prä- und intraoperativ der anteriore, posteriore und superiore Gelenkspaltraum jeweils rechts und links analysiert. Die individuellen Abstände zwischen den Messpunkten zeigen eine große Variabilität (vgl. Tabelle 13 min.-max.), die prä- und intraoperativ jedoch vergleichbar ist. Bei keiner der linearen Messung ergab sich eine signifikante Änderung zwischen den beiden Kondylenpositionen. Weder der anteriore (AJS) noch der superiore (SJS) oder posteriore (PJS) Gelenkspalt unterscheiden sich zwischen der präoperativen und intraoperativen Position.

Diese Ergebnisse lassen vermuten, dass die lineare Gelenkspaltmessung keine ausreichend genaue Aussagekraft bzgl. der Änderung der Gelenkposition hat.

Tabelle 13: Ergebnisse der linearen Gelenkspaltmessung

\begin{tabular}{lrrr}
\hline Messwert & Präoperative CR, median (min- & Intraoperative CR, median (min- & max $)$ \\
& $\max )$ & $5,5(3,7-13,0)$ & 0,712 \\
AJS-R (mm) & $6,2(3,4-13,4)$ & $5,4(1,7-9,4)$ & 0,657 \\
AJS-L (mm) & $5,5(1,9-9,4)$ & & $P$ \\
\hline
\end{tabular}




\begin{tabular}{lrrr}
\hline SJS-R (mm) & $3,9(1,7-7,1)$ & $4,0(2,0-8,7)$ & 0,922 \\
SJS-L (mm) & $4,1(1,7-6,7)$ & $4,1(1,5-6,8)$ & 0,686 \\
PJS-R (mm) & $5,7(2,2-11,7)$ & $5,5(2,3-11,8)$ & 0,945 \\
PJS-L (mm) & $4,4(1,0-8,5)$ & $4,1(1,1-8,3)$ & 0,871 \\
\hline
\end{tabular}

\subsection{Ergebnisse der volumetrischen Analyse}

\subsubsection{Volumetrische Ergebnisse des gesamten Probandenkollektivs}

Analysiert und volumetrisch verglichen wurden ausschließlich die vom Mandibulacorpus getrennten Kondylenköpfe jeweils rechts und links unabhängig voneinander.

Die volumetrischen Überlagerungen der Kondylenpositionen zeigten eine signifikante Änderung der Kondylenpositionen prä- und intraoperativ sowohl links als auch rechts (Tabelle 14, Abbildung 37). Die präoperative Position wurde mit 100\% angenommen. Im Mittel stimmte die intraoperative Kondylenposition mit der präoperativen Position rechtsseitig um 71,1\% überein, zeigte jedoch eine hohe individuelle Variation. Der $p$-Wert lag bei $<0,001$, nach Korrektur nach Bonferroni bei $p=0,015$ und ist damit hoch signifikant.

Der Volumenvergleich für die linke Kondylusposition ergab im Mittel eine Übereinstimmung von 60,6\%, auch hier zeigte sich eine große individuelle Variation. Der $p$ Wert lag bei $<0,001$, nach der Bonferroni-Korrektur bei 0,015 und war damit statistisch hoch signifikant.

Tabelle 14: Ergebnisse des volumetrischen Vergleiches der Kondylen

\begin{tabular}{lrrr}
\hline $\begin{array}{l}\text { Dreidimensionaler } \\
\text { Messwert }\end{array}$ & Volumetrische Übereinstimmung, \\
median (min-max) & $71,1(15,9-91,4)$ & $<0,001$ & $\begin{array}{r}\text { Bonferroni- } \\
\text { korrigiertes } P\end{array}$ \\
Volumen-R $(\%)$ & $60,6(23,3-91,7)$ & $<0,001$ & 0,015 \\
Volumen-L $(\%)$ & 0,015 \\
\hline
\end{tabular}




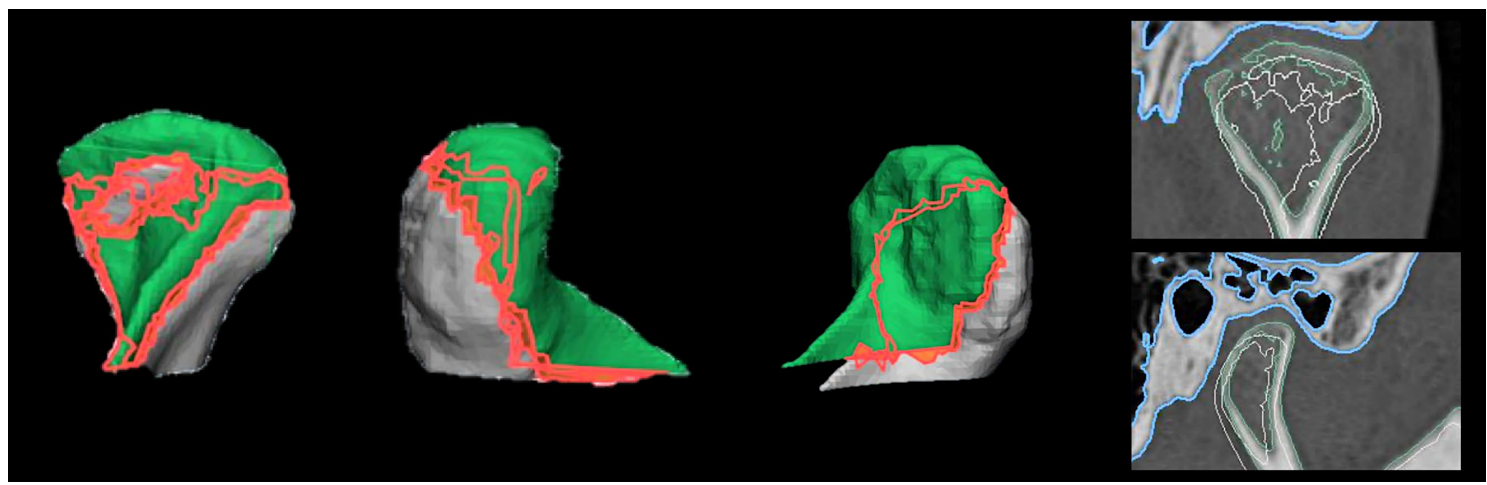

Abbildung 37: Kollidierendes Volumen der prä-und intraoperativen Gelenkposition und deren röntgenologische Darstellung (rechts).

Bei 19 der 30 Probanden ließ sich visuell eine inferior-posteriore Bewegung der Kondylen bei intraoperativer Registierung der Gelenkposition, wie in Abbildung 38 und Abbildung 39 dargestellt, ausmachen. Eine Korrelation zwischen der skelettalen Anomalie und der Bewegungsrichtung ließ sich nicht herstellen.

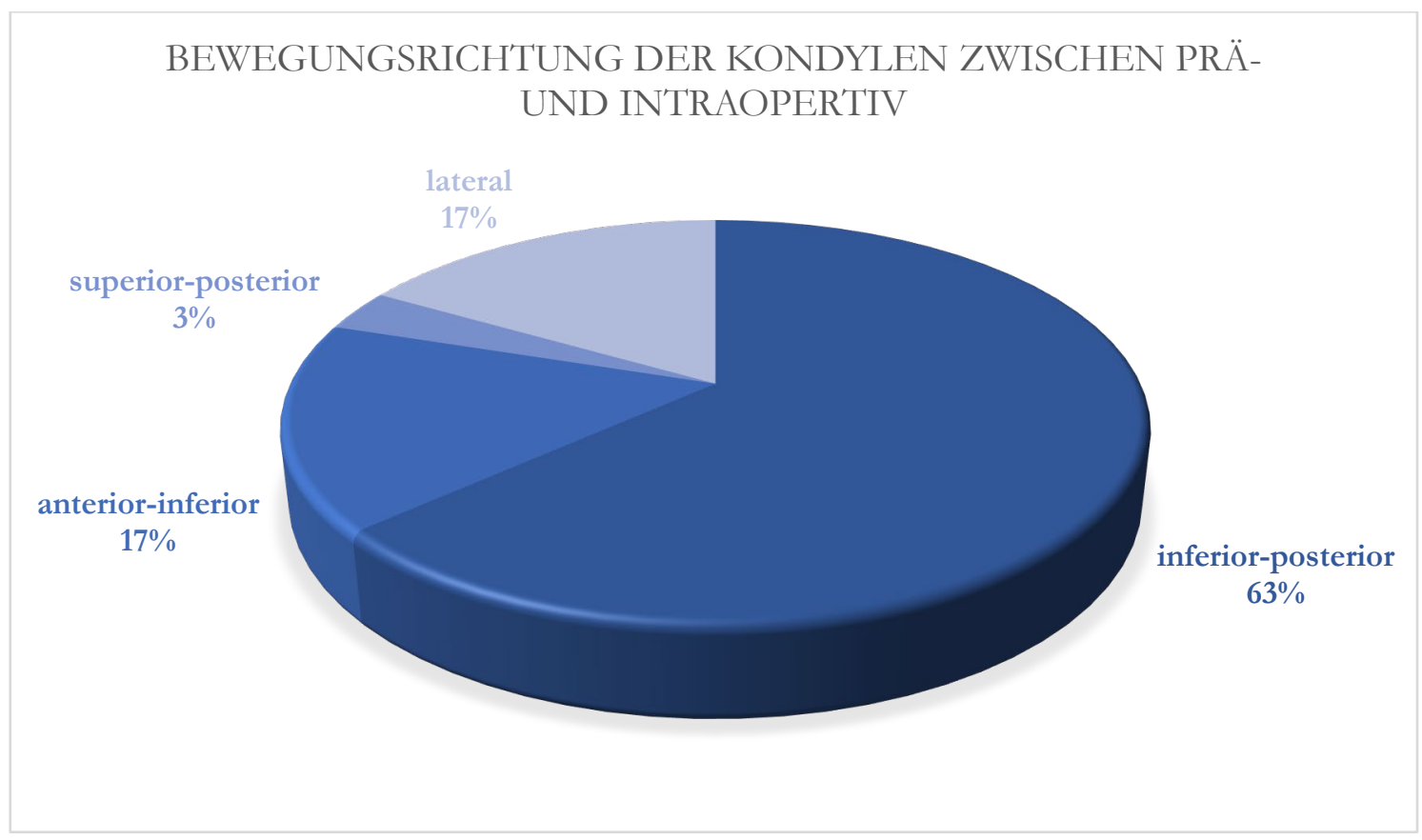

Abbildung 38: Visuelle Bewegungsrichtung der Kondylen zwischen prä-und intraoperativer Zentrik. Betrachtet wurden die Kondylenpaare pro Patient. 


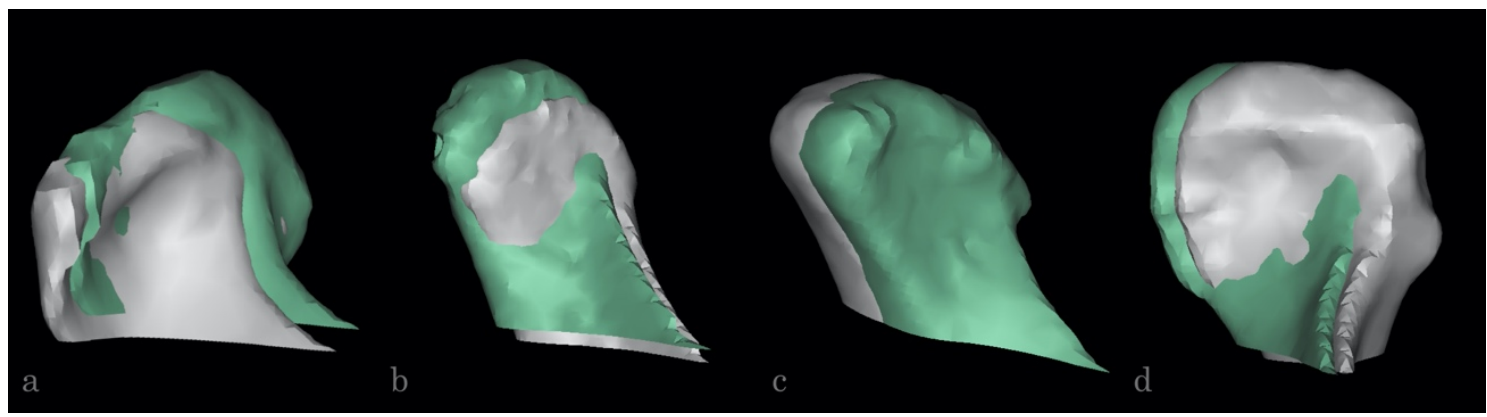

Abbildung 39: Bewegungsrichtung der Kondylen von präoperativ (grün) zu intraoperativ (grau) a) inferiorposterior b) anterior-inferior c) superior-posterior d) lateral.

\subsubsection{Ergebnisse der volumetrischen Subgruppenanalyse}

\subsubsection{Subgruppenanalyse in Bezug auf die Symmetrie}

Das Patientenkollektiv wurde anhand der Mentondeviation in symmetrische und asymmetrische Patienten aufgeteilt. Der Vergleich der volumetrischen Übereinstimmung zwischen den Subgruppen ergab weder für den linken noch für den rechten Kondylus einen signifikanten Unterschied (Tabelle 15).

Verglichen wurde zusätzlich die Übereinstimmung der Deviation - und der NonDeviationsseite bei symmetrischen und asymmetrischen Patienten. Auch der Vergleich der Deviationsseiten wies keine signifikanten Unterschiede auf.

Tabelle 15: Ergebnisse der volumetrischen Subgruppenanalyse Symmetrie

\begin{tabular}{lrrr}
\hline Dreidimensionaler Messwert & \multicolumn{2}{c}{ Volumetrische Übereinstimmung, median (min-max) } & $P$ \\
& Symmetrische Patienten & Asymmetrische Patienten & \\
& $70.9(15.9-86.0)$ & $74.0(28.7-91.4)$ & 0,397 \\
Volumen-R (\%) & $60.6(23.6-85.8)$ & $63.2(23.3-91.7)$ & 0,846 \\
Volumen-L (\%) & $71.2(15.9-85.1)$ & $62.8(23.3-91.7)$ & 0,681 \\
Volumen-Deviationsseite $(\%)$ & $58.9(23.6-86.0)$ & $70.1(48.4-89.4)$ & 0,109 \\
$\begin{array}{l}\text { Volumen-Nicht-Deviationsseite } \\
(\%)\end{array}$
\end{tabular}

\subsubsection{Subgruppenanalyse in Bezug auf die skelettale Klasse}

Die volumetrische Übereinstimmung der Kondylenpositionen wurde zwischen Patienten mit einer skelettalen Klasse II und Klasse III analysiert (Abbildung 40). Die Subgruppenanalyse 
ergab keine signifikanten Unterschiede zwischen den verschiedenen skelettalen Klassen (Tabelle 16).

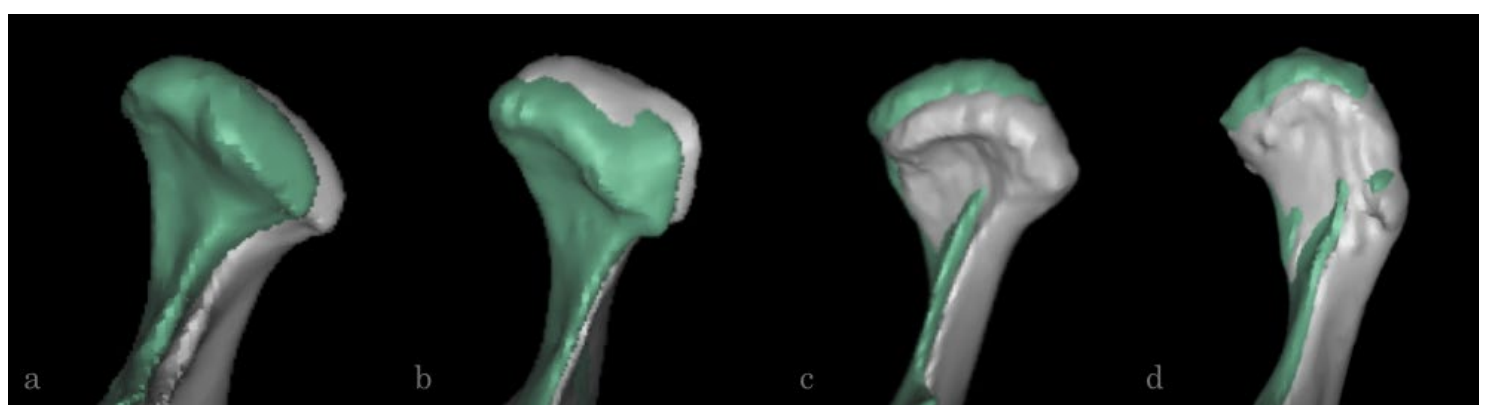

Abbildung 40: Darstellung der präoperativen (grün) und der intraoperativen (grau) Gelenkposition bei a) und

b) Klasse II Patienten und c) und d) Klasse III Patienten.

Tabelle 16: Ergebnisse der volumetrischen Subgruppenanalyse der skelettalen Klasse

\begin{tabular}{lrcr} 
Dreidimensionaler Messwert & Volumetrische Übereinstimmung & $P$ \\
& Skelettale Klasse II & Skelettale Klasse III & \\
& Median $($ Min - Max $)$ & Median $($ Min - Max $)$ & \\
Volumen-R (\%) & $71.3(15.9-85.7)$ & $72.0(28.7-91.4)$ & 0,698 \\
Volumen-L $(\%)$ & $59.8(23.6-85.1)$ & $68.6(23.3-91.7)$ & 0,423 \\
\hline
\end{tabular}




\section{Diskussion}

\subsection{Diskussion der Methodik}

Da die bisherigen Untersuchungen zur Kondylenposition bei Dysgnathiechirurgie auf Basis dentoalveolärer Relationen anhand von Gipsmodellen und Artikulatoren durchgeführt wurden (Bamber et al. 1999; Yaghmaei et al. 2013; Zak et al. 1992), gibt es bis dato keine vergleichbare, empirische Methodik, die durch vorherige Studien validiert wurde.

\subsubsection{Definition der Zentrik}

Die Definition der zentrischen Kondylenposition wurde intensiv in der Literatur diskutiert. Die Acadamy of Prosthodontic (2005) gibt in ihrem Glossar der prothetischen Begriffe, sieben mögliche Definitionen der zentrischen Relation an. Einigkeit bzgl. der Zentrik herrscht also nicht einmal innerhalb einzelner Fachrichtungen der Zahnmedizin. Da die physiologische Fossa-Kondylen-Beziehung jedoch in nahezu allen zahnmedizinischen Disziplinen von Relevanz ist, wurden zahlreiche Definitionen und Positionierungsmöglichkeiten aufgezeigt.

Die Definition der zentrischen Kondylenposition hat sich im letzten Jahrhundert stetig gewandelt. Hanau (1929) definierte die Zentrik als die Position, an der die Kondylen auf den Disken in der Gelenkpfanne ruhen, unabhängig von der Mundöffnung. In den darauffolgenden vier Dekaden wurde die Zentrik meist als die am retralsten zu positionierende Lage beschrieben (McCollum 1960; Thompson 1946). Zu Beginn der 70er Jahre existierten an die 40 mögliche Definitionen (Becker et al. 2000). Während Dawson (1979) erstmalig die Zentrik als superiorste Position der Kondylen beschrieb, entgegnete Smith Jr (1975), dass die retralste Position mit dem Gothischen-Bogen/Pfeilwinkelregistrat gesichert werden kann und die präziseste Positionierungsmöglichkeit bietet. Bis zum heutigen Zeitpunkt kam eine Vielzahl an Beschreibungen hinzu, von retralen über möglichst superiore, bishin zu anterioren und lediglich schmerzfreien Positionen (Palaskar et al. 2013).

In einer Literaturübersicht sprechen Rinchuse und Kandasamy (2006) von einem Wandel der Definition von der retralsten in eine anterior-superiore Position, wobei die Autoren zu bedenken geben, dass jede vom Behandler manipulierte Position nicht der physiologischen entspricht. Auch Keshvad und Winstanley (2001) geben zu bedenken, dass Uneinigkeit bei der Registrierung der Zentrik herrscht und keine abschließende Aussage getroffen werden kann. Wichtige Schlussfolgerungen ihrer Untersuchung sind die Wiederholbarkeit der Position und die möglichst drucklose und nicht forcierte Zentriknahme.

Eine eindeutige Beurteilung wie die zentrische Kondylenposition genommen werden soll, geschweige denn definiert werden kann, steht also noch aus. In der vorliegenden Studie wird, ähnlich wie von Christensen (2004) beschrieben die „komfortable, posteriore Position der 
Mandibula“ die mit einem gleichmäßigen, leichten Druck nach distal erreicht werden kann. Laut Autor ist diese Art von Registrierung reproduzierbar und für die meisten Behandler akzeptabel. Wie bereits erwähnt orientiert sich die Studie zusätzlich an der offiziellen Definition der DGFDT, der „kranio-ventralen, nicht seitenverschobenen Position beider Kondylen bei physiologischer Kondylus-Diskus-Relation und physiologischer Belastung der beteiligten Gewebestrukturen“ (DGZMK 2005).

Da auf Basis der Literatur die Beschreibung der Zentrik und die damit zusammenhängende Registrierung immer noch großes Diskussionspotential bietet, spricht die Orientierung an einer offiziellen Definition für eine vertretbare und nachvollziehbare Variante. Zudem erhebt die Studie nicht den Anspruch, eine neue Positionierung der Kondylen zu untersuchen. Im Gegenteil - bei dem untersuchten Patientenklientel in dieser Studie war die Ausgangsbasis die Kondylenposition zu erhalten. In Bezug auf die Methodik und die wiederholte Zentriknahme an einem Patienten unter unterschiedlichen Voraussetzungen, ist eine repositionierbare Grenzposition unabdingbar für die Durchführung dieser Studie, die bei der retralen, drucklosen Positionierung gegeben ist.

\subsubsection{Erfassung der Gelenkposition}

Die Erfassung der zentrischen Kondylenposition wird in der Literatur als auch im klinischen Alltag höchst variabel durchgeführt. In dieser Studie wurde sich an dem klinischen Behandlungsablauf der Abteilung für Kieferorthopädie der UMG orientiert. Mithilfe des Kinnführungsgriff nach Lauritzen (Lauritzen 1974) wird der Patient drucklos in die retralste Position der Zentrik geführt. Diese Position ist leicht zu repositionieren und bietet damit eine wiederholbare und vergleichbare Registrierungsmöglichkeit.

Da alle Zentrikregistrate, sowohl prä- als auch intraoperativ durch einen Behandler durchgeführt wurden, lassen sich individuelle Fehler minimieren. Die Reproduzierbarkeit von Bissnahmen ist besondere im prothetischen Bereich erforscht. Studien zur Registrierung bei Totalprothesenpatienten zeigen zwar bedingt durch große Standardabweichungen keine signifikanten Unterschiede zwischen Behandlern, merken jedoch die hohe Adaptationsfähigkeit der Patienten an die neue Situation und das fachliche Können des Behandlers an (Kühl 1967; Utz et al. 1993; Utz et al. 2002). Dagegen konnte bei der Zentriknahme von bezahnten Patienten ein signifikanter Unterschied der Mandibulaposition zwischen Behandlern festgestellt werden (Etz et al. 2012; Lentner et al. 1997). Die Autoren wiesen zudem darauf hin, dass sich die elektromyografische Aktivität besonders im Musculus temporalis durch die behandlerindividuelle Zentrikpositionierung stark unterschied.

Im Rahmen der hier vorliegenden Studie wurde die präoperative zentrische Kondylenposition anhand des DVTs nochmals für alle Probanden visuell bestätigt.

Ein weiterer relevanter Aspekt, der eine sagittale Diskrepanz vortäuschen kann, ist die vertikale Bisssperrung. Innerhalb der Studie wurden alle Zentrikregistrate dünn gestaltet um 
eine vertikale Diskrepanz so gering wie möglich $\mathrm{zu}$ halten. Bedingt durch die Materialeigenschaften ist eine minimale vertikale Sperrung unumgänglich. Jedoch betragen im Mittel die Dicken der prä- und intraoperativen Registrate am dünnsten Punkt nur 0,4mm bis $0,46 \mathrm{~mm}$. Auf die Kondylenposition hat dies wahrscheinlich keine klinische Relevanz. Zudem wiesen die prä- und intraoperativen Registrate eine vergleichbare Dicke auf, ein patientenindividueller Fehler lässt sich also ausschließen.

Die Erfassung der Zentrik ist also für die Studienziele und die statische Auswertung auf Basis dieser Gelenkpositionen geeignet.

\subsubsection{Problematik des Datensatzes und der Rekonstruktion}

Die gesamte Analyse der Studie basiert auf den röntgenologischen Datensätzen einer DVT mit der Voxelgröße von 0,3mm. Laut Costa et al. (2018) und Whyms et al. (2013) ist diese Voxelgröße ausreichend für volumetrische Untersuchungen. Dennoch ist eine geringe Fehlerquote bedingt durch Ungenauigkeiten im DVT nicht ausgeschlossen. Besonders die Rekonstruktion der Kondylen ist abhängig von der Genauigkeit des DVTs. Zudem kommt die händische Nachbearbeitung mit individuell angepassten threshold, der je nach Untersuchung variieren kann. Die innerhalb der Studie durchgeführte Interrater-Reliabilität zeigt jedoch, dass nur eine minimale Abweichung innerhalb der beiden Untersucher vorliegt. Maximale Abweichung sind an Grenzpunkten bis zu 1,5mm, im Mittel jedoch nur 0,18mm. Diese mittlere Abweichung scheint klinisch vernachlässigbar zu sein, sollte jedoch bei den Ergebnissen berücksichtigt werden. Es ist also davon auszugehen die rekonstruierten Kondylen stimmen nicht vollständig mit den natürlichen Kondylen überein.

Da für die Studie zwar zwei Kondylenpositionen verglichen werden, jedoch auf Basis nur einer Rekonstruktion, ist selbst ein potentieller Fehler irrelevant. Diese Rekonstruktion erfolgt anhand des präoperativen DVTs und wird nur einmalig für jeden Patienten durchgeführt. Die Kondylen der prä- und intraoperativen Zentrik sind in ihrer Dimension und ihrem Volumen vollständig identisch. Ein methodischer Fehler diesbezüglich kann also ausgeschlossen werden.

Zudem ist die Studie unabhängig von Fehlerquellen durch Aufnahmegeräte, da alle genutzten Datensätze von einem DVT-Gerät stammen.

\subsubsection{Fehlerquellen und Problematik der cephalometrischen Analyse}

Das FRS hat sich als Standard in der kieferorthopädischen Behandlung und Diagnostik schon lange etabliert und bietet durch die einfache Durchführung und gute Qualifizierbarkeit der Analyse (Bell und Jacobs 1981; Wallen et al. 1986) ein bisher optimales vergleichendes Mittel der skelettalen Gegebenheiten. Die klassische cephalometrische Analyse bietet die Auswertungsmöglichkeiten in sagittalen und vertikalen Dimensionen, die transversale Dimension kann aufgrund der zweidimensionalen Aufnahme jedoch nicht beurteilt werden. 
Diese darf jedoch bei dem komplexen Aufbau des Gesichtsschädels, besonders bei Dysgnathieoperationen nicht vernachlässigt werden.

Bereits die zweidimensionale Auswertung des FRS ist fehlerbehaftet. So liegen potentielle Fehlerquellen sowohl in Projektionsfehlern des Röntgenbildes, als auch bei Identifikationsfehlern der anatomischen Strukturen und dadurch bedingte Messfehler (Baumrind und Frantz 1971).

Diese Fehlerquellen sind auch bei der dreidimensionalen cephalometrischen Auswertung relevant. Zwar können Projektionsfehler durch Überlagerung mehrerer Strukturen ausgeschlossen werden, jedoch sind einige Messpunkte anhand von anatomischen Strukturen deutlich schwieriger zu setzen und weisen dadurch eine hohe Variabilität auf. Beispielhaft zu nennen ist der posteriore Gelenkspalt (PJS): selbst in der IntraraterReliabilität zeigt sich eine große Varianz der Messwerte. So war die mittlere Differenz bei dem präoperativen PJS rechts zwar nur -0,08 mm, weicht aber im Minimum um -2,31 und im Maximum um $+2,47 \mathrm{~mm}$ ab. Dies bedeutet eine Range von 4,78mm, die nicht mehr zu vernachlässigen ist. Mögliche Ursachen für diese hohe Abweichung sind die individuell teilweise stark unebenen Rekonstruktionen der knöchernen Strukturen der distalen Kondylusoberfläche und der korrespondierenden Fossa. Durch die dichten weichgewebigen Strukturen im Gelenkspalt, lassen sich bei der Rekonstruktion der Gelenkoberfläche die ossären Strukturen nicht klar abgrenzen von Geweben mit einem ähnlichen HU-Wert. Dieses bedingt eine teilweise raue Oberfläche, besonders im Bereich der distalen Kondylusbereiche. Hinzu kommt die Positionierung des Messpunktes: Der Messpunkt muss im Gegensatz zur klassischen zweidimensionalen Analyse in allen Ebenen korrekt und repositionierbar gesetzt werden. Durch die zusätzliche dritte Ebene lässt sich z. B. der distalste Kondyluspunkt sehr variabel setzen. Da dieser Messpunkt zudem keine klar definierte anatomische Struktur wiederspiegelt, ist hier eine vorherige Konkretisierung im Rahmen der Cephalometrie unabdingbar. Laut Scapino (1997) weisen Kondylen zudem eine hohe Variabilität in Form und Größe auf, sodass die standardisierte Setzung der Messpunkte schwierig ist. Bedacht werden muss auch, dass die Software ProPlan ${ }^{\circledR}$ CMF 3.0 die kürzeste Strecke zwischen den Messpunkten misst. Weichen die Markierungen jedoch ungleichmäßig stark in der Vertikalen oder Transversalen voneinander ab, werden die Werte deutlich größer und spiegeln nicht mehr die reine sagittale Dicke des Gelenkspaltes wieder. Ähnliche Werte ergeben sich jedoch auch bei den Messungen auf der intraoperativen Maske, sodass man davon ausgehen kann alle Punkte wurden gleich schlecht oder gut gesetzt.

Wie bereits erwähnt, sind die Streckenmessungen zwar prä- und intraoperativ mit einander vergleichbar, spiegeln aber nicht zwangsläufig die reale klinische Situation wieder. Alle Strecken verlaufen dreidimensional durch den Raum, sind also räumliche Vektoren. Sie geben keine Auskunft über die tatsächliche Distanz in einer Ebene. So wie für den PJS erläutert, ist der PJS-r-Wert keine reine sagittale Messung des Gelenkspaltes. Selbiges gilt für 
alle anderen dreidimensionalen Werte. Ausgenommen sind die klassischen FRS-Werte zur Kategorisierung der Patienten, die entlang einer konstruierten Ebene verlaufen um eine Vergleichbarkeit mit prä- und postoperativen FRS zu gewährleisten. Laut Zamora et al. (2012) zeigen dreidimensionale cephalometrische Analysen eine sehr geringe Fehlerquote $(1,5 \%)$ und besonders Landmarken wie Nasion, Sella, A-Punkt und Menton zeigen eine gute Reliabilität bei der Setzung. Olmez et al. (2011) sehen sogar eine Überlegenheit der dreidimensionalen zur konventionellen zweidimensionalen cephalometrischen Vermessen. Hingehen wenden Pittayapat et al. (2014) ein, dass die aktuelle Datenlage zu dreidimensionalen cephalometrischen Analysen noch nicht ausreichend ist für eine systematische Überprüfung.

Zwar wurde durch die Auswertung nur eines Untersuchers die individuelle Fehlerquote reduziert, jedoch besteht bei der cephalometrischen Auswertung ein deutlich erhöhtes Risiko eines systematischen Fehlers.

\subsection{Diskussion der Ergebnisse}

\subsubsection{Patientenkollektiv}

Ausgewertet in dieser Studie wurden die Kondylenpositionen links und rechts zu zwei Untersuchungszeitpunkten von 30 Probanden, die sich in der UMG einer Dysgnathieoperation unterzogen. Es handelt sich um 16 weibliche und 14 männliche Probanden. Das Verhältnis ist damit nahezu ausgeglichen.

In den Untersuchungen von Simpson (1974) und Michel (1990) geben die Autoren bzgl. des Patientengutes von Dysgnathieoperationen eine Verschiebung des Verhältnisses deutlich zur weiblichen Seite an. Auch Proffit et al. (1990) gibt ein Verhältnis von weiblichen zu männlichen Patienten von 2:1. Das Patientengut der Studie ist damit nicht übereinstimmend mit den bisherigen Erkenntnissen der Literatur und könnte damit nicht repräsentativ für die Gesamtheit der Dysgnathiepatienten sein. Laut Michel (1990) gebe es keine Anhaltspunkte auf genetischen oder ätiologischen Faktoren, die dieses Phänomen der ungleichen Verteilung der Geschlechter erklärt. Der Autor geht daher davon aus, dass weibliche Patienten eher dazu neigen, aus ästhetischen und psychosozialen Gründen den Eingriff auf sich zu nehmen. Da sich die Studie jedoch ausschließlich auf die Position der Kondylen bezieht und keine Differenzen zwischen weiblichen und männlichen Patienten zu erwarten ist, kann in Betrachtung des eingeschränkten Studienzieles das Patientenkollektiv als repräsentativ angenommen werden.

Die Verteilung der skelettalen Klassen ist ebenfalls ausgeglichen zwischen den Klassen II $(n=13)$ und III ( $n=14)$. Unterrepräsentiert ist die skelettale Klasse I $(n=3)$, die daher nicht in der Subgruppenanalyse berücksichtigt wurde. Da es sich bei den Probanden der Studie um 
Patienten handelt, die eine Indikation zur kombiniert kieferorthopädisch-kieferchirurgischen Therapie haben, erscheint die geringe Anzahl an Klasse I Patienten nicht unwahrscheinlich. Die Behandlungsnotwenigkeit ergibt sich bei diesen Patienten durch die vertikale Diskrepanz der Kieferbasen im Sinne eines offenen Bisses. Das Probandenkollektiv ist damit repräsentativ und ermöglicht eine Subgruppenanalyse in Bezug auf die Verteilung der skelettalen Anomalien.

Da die Dysgnathiechirurgie skelettale Anomalien der Kieferlage therapiert, ist das endgültige Ausmaß der Fehlbildung erst nach Wachstumsabschluss ersichtlich. Daher sollte der Operationszeitpunkt immer nach Abschluss des Wachstums gewählt werden, um die Langzeitstabilität des Ergebnisses zu sichern. Schwenzer und Ehrenfeld (1990) sehen ein abgeschlossenes Wachstum als unumgängliches Erfordernis. Kallela et al. (1998) geht sogar zur Prophylaxe von Rezidiven bei Unterkiefervorverlagerung von einem Durchschnittsalter von 27 Jahren aus. Laut Puelacher und Waldhart (1987) liegt das Operationsalter bei Progenieoperationen zwischen dem 20. und 30. Lebensjahr. Das in dieser Studie untersuchte Patientenkollektiv ist im Durchschnitt 25,8 Jahre alt und ist damit übereinstimmend mit den Ergebnissen der Literatur.

Jedoch ist zu betrachten, dass das Patientengut mit 30 Probanden relativ klein ist und keine Formulierung von allgemeingültigen Aussagen auf Basis der Studienresultate möglich ist. Für aussagekräftigere Ergebnisse müssten weiterführende Studien ein größeres Kollektiv einbeziehen.

\subsubsection{Cephalometrischen Ergebnisse}

Insgesamt wurden Positionen von 60 Kondylen zu zwei unterschiedlichen Zeitpunkten präoperativ und intraoperativ - untersucht. Keine der linearen Messungen hat eine statistisch signifikante Änderung der Kondylenposition ergeben. Wie bereits beschrieben, unterliegen die linearen Messungen einer potentiell hohen Fehlerquelle. Anhand der Messwerte lässt sich jedoch eine Vergleichbarkeit der beiden Positionen annehmen: Der Wert Ii-Approx-SAG (mm) zur Bestimmung der Mittellinienabweichung ist im Median präoperativ 0,6mm groß, intraoperativ 0,5mm. Ähnlich nahe Beziehungen bestehen auch zwischen den prä-und intraoperativen der Gelenkspaltmessung und der anderen dentalen Messpunkte. Sollte die Hypothese der erhöhten Fehlerquelle zutreffen, wäre eine stärkere Abweichung der Messwerte zu erwarten. Es ist daher davon auszugehen, dass die linearen Messwerte unter den Zeitpunkten vergleichbar sind, die Änderung der Kondylenpositionen jedoch nicht ausreicht, um statistisch detektiert zu werden.

Besonders die dentalen Werte sind diesbezüglich interessant. So lässt sich weder bei der Mittellinienabweichung, noch bei dem Verhältnis der korrespondieren Molaren zueinander eine Änderung feststellen. Laut Ellis (1994) und Costa et al. (2018) sind Hilfsmittel zur Positionierung der Kondylen im klinischen Alltag immer noch nicht etabliert. Ellis (1994) 
gibt als Gründe den erhöhten Zeitaufwand bei der Herstellung solcher Hilfsmittel und das Können des Chirurgen die Kondylen selbst zu positionieren an. Da die Änderungen auf dentaler Ebene laut Studienergebnissen so gering ausfallen und für die Positionierung der Kondylen normalerweise die Okklusion als Referenz genommen wird, können so Änderungen auf kondylärer Ebene für den Operateur unbemerkt bleiben. Auf Basis der Studie von Zak et al. (1992) mit dem Ergebnis, dass Barbiturate, die während der Narkose zum Einsatz kommen, ausreichend sind um eine kondyläre Positionsänderung hervorzurufen, besteht also ein erhöhtes Risiko einer fehlerhaften und zugleich unbemerkten Fehlpositionierung, wenn keine Hilfsmittel zur Anwendung kommen. Die klinische Relevanz minimaler okklusaler Interferenzen ist jedoch fragwürdig, da das Kiefergelenk sehr anpassungsfähig ist.

Eine Untersuchung von Bicaj et al. (2013) befasst sich mit der Auswirkung einer digitalen Okklusionsfolie auf die Position der Kondylen bei maximaler Interkuspidation. Die T-ScanFolie (T Scan III, TekScan, Bosten, USA), die von den Autoren untersucht wurde, ist lediglich $100 \mu \mathrm{m}$ dick und sorgte bereits für eine signifikante Änderung der Kondylenposition, die mittels Ultraschall-Axiographigerät beurteilt wurde. Es kam bei Anwendung der Folie zu einer Ventalverschiebung um ca. 1mm der Kondylen.

Eine okklusale Differenz von so geringem Ausmaß ist visuell im Patientenmund nicht detektierbar und dennoch kommt es, laut der Untersuchung von Bicaj et al. (2013), zu einer Verzehnfachung der Veränderung in der Gelenkposition.

Dies bedeutet, dass auch die nicht statistisch signifikanten, dentalen Messwerte dieser Studie zu deutlichen Positionsverschiebungen der Gelenke führen. Wie bereits erwähnt, findet die intraoperative Gelenkpositionierung häufig ohne Hilfsmittel statt und wird lediglich anhand der Okklusion durchgeführt. Wenn davon auszugehen ist, dass eine potentielle Veränderung auf dentaler Ebene zu einer Verstärkung um eine Zehnerpotenz der kondylären Verhältnisse führt, wäre folglich eine exakte Positionierung der Kondylen unmöglich. Fragwürdig ist jedoch, in wie weit eine Untersuchung zur maximalen Interkuspidation Rückschlüsse auf die Zentrik zulässt.

Außer Acht sollte auch nicht die Bewegung der Kondylen, die Kondylenbahn, gelassen werden. Verallgemeinert wird die Dynamik der Gelenke häufig als Rotation und Translation beschrieben und im Laufe der Geschichte vielseitig untersucht, besonders im Aspekt der individuellen Einstellung von Artikulatoren im prothetischen Bereich oder der Funktionstherapie. Zum Verständnis des Kiefergelenkes, seiner Funktion und deren Konsequenz für den zahnmedizinischen Alltag haben besonders Prothetiker wie Gysi, Gerber oder Lauritzen beigetragen. Die Bezeichnung des Kiefergelenkes als Dreh- und Gleitgelenk ist allgemein anerkannt und vereinfacht die Visualisierung.

Geht man von einer hauptsächlichen Rotationsbewegung bei initialer Kieferöffnung aus, könnte man annehmen, kleinere okklusale Unterschiede werden durch die Rotation 
kaschiert. In der Literatur sind Angaben zu finden, dass es aus der retralen Kontaktposition in den ersten $20 \mathrm{~mm}$ der Kieferöffnung zu keiner translatorischen Bewegung kommt (Lehmann et al. 1998). Maeda et al. (1992) und Ferrario et al. (1996) geben jedoch an, dass zu jeder Zeit der Bewegung eine rotatorische und translatorische Komponente vorhanden ist. Zudem weisen Kordaß und Ruge (2015) darauf hin, dass bisherige Untersuchungsmethoden zur Bewegungsaufzeichnungen das Rotationszentrum nur annäherungsweise bestimmen können und eine reale Darstellung der Dynamik nicht möglich ist. Die Autoren empfehlen eine patientenindividuelle Bestimmung über eine Kombination aus einer DVT und ultraschallbasierten Bewegungsaufzeichnungen.

Ob geringfügige okklusale Differenzen klinische Relevanz auf die Funktion der Kondylen haben, lässt sich noch nicht beurteilen und muss langfristig noch evaluiert werden.

\subsubsection{Volumetrische Ergebnisse}

Die dreidimensionale volumetrische Überlagerung der 60 Positionen wurde auf Basis der kollidierenden Volumina der Kondylen analysiert. Sowohl für die rechten als auch linken Kondylen zeigten sich signifikante Änderungen von der präoperativen zur intraoperativen Gelenkstellung mit einer hohen Variation der Prozentwerte. Auf der rechten Seite stimmten die Positionen im median um 71,1\% überein, auf der linken Seite nur um 60,6\%. Die Bewegung im linken Kondylus war damit laut dieser Studie deutlich größer als die des rechten Kondylus. Beide Ergebnisse waren mit einem p-Wert von <0,001 (nach BonferroniKorrektur 0,015) hoch signifikant.

Die hier gezeigten Ergebnisse stimmen mit bisherigen Analysen auf dentaler Ebene aus der Literatur überein. So berichten auch Zak et al. (1992), Yaghmaei et al. (2013) und Bamber et al. (1999) von einem signifikanten Unterschied der Kondylenposition unter Einfluss von Narkotika. Die bisherigen Studien unterscheiden sich in der Methodik jedoch gravierend von der hier gezeigten und basieren auf linearen, dentoalveolären Messungen.

Reyneke und Ferretti (2002) untersuchten an 184 Patienten die okklusalen Verhältnisse zum Operationszeitpunkt und eine Woche postoperativ. Die Bissregistrierung erfolgte mithilfe eines Wachsbisses. Bereits intraoperativ, nach Entfernung der intermaxillären Fixation wurde bei 18 Patienten eine inkorrekte Positionierung festgestellt, bei 15 Patienten wurde sieben Tage postoperativ ein Absinken des Kondylus festgestellt von denen 3 Patienten eine fehlerhafte Okklusion entwickelten. Zu beachten ist, dass die Untersuchung keine präoperativ hergestellten Positionierungshilfen nutzt und ein Wachsbiss nur geringfügig formstabil ist. Ein methodischer Fehler lässt sich bei dieser Untersuchung also nicht ausschließen.

Yaghmaei et al. (2013) nutzten einen semi-adjustierbaren Artikulator und Gipsmodelle um die Auswirkung einer Generalanästhesie auf die Zentrik zu analysieren. Von 50 Patienten wurden drei verschiedene okklusale Registrate angefertigt: präoperativ aufrecht und im 
Liegen, sowie intraoperativ im Liegen. Mit einer Messlehre wurden die Unterschiede der okklusalen Verhältnisse auf $0,1 \mathrm{~mm}$ Genauigkeit vermessen. Besonders Patienten mit einer skelettalen Klasse II Anomalie zeigten signifikante Veränderungen unter der Einwirkung einer Allgemeinanästhesie. Auch die Lagerung des Patienten zeigte signifikante Auswirkung auf die Okklusion.

Auch Zak et al. (1992) verwendeten okklusale, in einen Artikulator übertragene Registrate. Pro Probanden wurden drei Registrate angefertigt - präoperativ im wachen Zustand, intraoperativ im narkotisierten und im narkotisierten, relaxierten Zustand. Bei der Untersuchung von 10 Patienten konnte ein Einfluss der Generalanästhesie auf die okklusalen Verhältnisse festgestellt werden. Der Einsatz von Muskelrelaxantien zeigte jedoch bei keinem Probanden eine zusätzliche Auswirkung.

Borba et al. (2014) konnten bei der Untersuchung von 30 Patienten keinen Einfluss der Narkose auf die zentrische Okklusion finden. Die prä- und intraoperativen okklusalen Registrate wurden mithilfe von Gipsmodellen einartikuliert und anatomische Referenzpunkte zur Vermessung gesetzt. Anhand der Ergebnisse schließen die Autoren darauf, dass eine intra-operative, manuelle Positionierung der Zentrik möglich ist.

Die Vorgehensweisen der genannten Studien sind also ähnlich und die Ergebnisse untereinander vergleichbar. Da die Methodik der vorliegenden Untersuchung sich jedoch deutlich unterscheidet, lassen sich die Resultate nur bedingt mit der bisherigen Literatur ins Verhältnis setzen.

Im Gegensatz zu Yaghmaei et al. (2013) konnte in der Subgruppenanalyse kein signifikanter Unterschied zwischen Patienten mit Klasse II und III festgestellt werden. Dies könnte an den verschiedenen Klassifikationen der Patienten liegen: während Yaghmaei et al. (2013) die Probanden auf dentaler Ebene einteilt, nutzt diese Studie die skelettalen Relationen. Eine weitere Erklärung für diese differenten Ergebnisse, könnte die atypische Muskelfunktion bei ausgeprägten skelettalen Anomalien sein (Thilander et al. 2002). Besonders Klasse II Patienten, die über die Muskelfunktion eine Kompensation der Kieferbasisdifferenz zu erreichen versuchen, zeigen bei der Zentriknahme eine erschwerte Führung. Weffort et al. (2010) weisen darauf hin, dass die zentrische Kondylenposition meist retraler als die maximale Interkuspidation liegt, laut Tripodakis et al. (1995) sogar retraler als die neuromuskuläre Position der Mandibula. Da in der Studie die Registrierung der Zentrik nur bei vollständiger Entspannung des Patienten erfolgte, könnte der nicht signifikante Unterschied zwischen den skelettalen Klasse II und III an der exakten präoperativen Registrierung liegen.

Zudem wurden in dieser Studie alle Zentrikregistrate von einem Behandler durchgeführt. Wie Etz et al. (2012) bereits feststellten ist der individuelle Behandlereinfluss sogar bei praxiserprobten Zahnärzten deutlich. Da ein Ein-Behandler-Szenario im klinischen Alltag nicht realistisch ist, ist davon auszugehen, dass sich die Kondylenpositionen bei einer 
Vielzahl unterschiedlicher Behandler deutlich stärker unterscheiden. Besonders bei unerfahrenen Behandlern, bei denen die Registrierung der Zentrik nicht zur alltäglichen Praxis gehört, sind bedeutend größere Unterschiede zu erwarten. Die Wahrscheinlichkeit einer Fehlpositionierung der Zentrik und das Risiko postoperativer Komplikationen steigt damit.

Der Einsatz von Hilfsmitteln zur Positionierung scheint somit, besonders für unerfahrene Behandler als sinnvoll, um die von Ueki et al. (2007) und Chen et al. (2013) postoperativen Änderungen der Kondylenposition so gering wie möglich zu halten und ein stabiles, vorhersehbares Ergebnis zu erzielen (Rotskoff et al. 1991).

Die Unterschiede der Kondylenpositionen haben nicht nur Auswirkungen auf die postoperative Gelenkstellung, sondern auch auf die Langzeitstabilität des chirurgischen Ergebnisses (Eggensperger et al. 2006; Ellis und Hinton 1991). Wie bereits erwähnt gehört die CMD zur zweithäufigsten postoperativen Komplikation nach Nervschädigungen (Jędrzejewski et al. 2015), besonders weibliche Patientinnen mit Klasse II gehören diesbezüglich zu Risikopatienten (Jędrzejewski et al. 2015; Toll et al. 2010). Bei der Geschlechterunterscheidung geben jedoch alle Autoren zu bedenken, dass Frauen häufig sensitiver auf Symptomatik reagieren und häufiger ärztlichen Rat aufsuchen als Männer. Eine anatomische Grundlage bestehe nicht. Als Gründe für skelettale Rezidive oder postoperative CMD werden das Absinken des Kondylus (,sagging condyle“), inkorrekte Positionierung des proximalen Segmentes, muskuläre oder ligamentäre Interferenzen und intraartikuläre Ödeme oder Blutergüsse bevor die Kieferanteile fixiert werden, genannt.

Bemerkenswert ist jedoch, dass Toll et al. (2010) in einer MRT-Untersuchung von 58 Dysgnathiepatienten deutliche Hinweise auf das vermehrte Vorkommen von präoperativen Kiefergelenkspathologien bei Klasse II und III Patienten fanden. Besonders Klasse-IIPatientinnen zeigen einen hohen Anteil an dorsal verlagerten Kondylen und ventral verlagerten Disken mit anterioren Prolaps. Zur genaueren postoperativen Beurteilung von degenerativen Prozessen oder Fehlpositionierung der Gelenke müsste idealerweise eine präoperative MRT als Referenz betrachtet werden. Die Autoren geben weiterhin zu bedenken, dass durch die häufige Überkorrektur nach dorsokranial bei der intraoperativen Kondylenpositionierung zur Prävention eines „sagging condyle“, vermehrt CMDSymptomatiken entwickelt werden. Auch die bereits erwähnte Studie von Claus et al. (2019) zeigt verstärkte Resorptionsvorgänge im Bereich des superioren und posterioren Gelenkspaltes bei händischer, intraoperativer Positionierung der Kondylen, was man als einen Hinweis auf die Spätfolgen der Überkorrektur interpretieren kann. Im Rahmen der Untersuchung kamen zwar intraoperative Positionierungshilfen zum Einsatz, es findet sich jedoch kein Hinweis auf eine Kondylenpositionierung mithilfe eines Splintes sondern es fand lediglich eine spannungsfreie Anpassung der Osteosyntheseplatten zwischen dem gelenkund zahntragendem Anteil der Mandibula statt. 
Die vorliegende Studie zeigt nur eine Momentaufnahme der zentrischen Kondylenpositionierung. Das kleine Patientenkollektiv ist zwar statistisch aussagekräftig bzgl. der Fragestellung, dennoch ist eine weitergehende Untersuchung sinnvoll. Zusätzlich wäre eine Untersuchung der definitiv erreichten Kondylenposition notwendig. In diesem Zusammenhang sind dreidimensionale Röntgenbilder, die während der Operation oder in einem ausreichenden Abstand postoperativ angefertigt werden, untersuchungswert. Das in der UMG fünf Tage postoperativ durchgeführte DVT ist durch die ödematöse Schwellung im Gesichtsbereich und die damit verbundene Veränderung der Gelenkposition nur bedingt für die Untersuchung geeignet. Eine langfristige Begleitung des Probandenkollektives kann zudem Spätfolgen wie skelettale Rezidive oder CMD aufzeigen. Die in der Studie registrierte Zentrik wurde zwar visuell und taktil als zentrische Kondylenposition verifiziert, dennoch steht eine klinische Evaluierung aus. Fraglich ist also, wie präventiv sich diese Position im klinischen Verlauf auf postoperative Rezidive oder Gelenkbeschwerden auswirkt und wie gut diese Position letztendlich mithilfe der Splinte übertragen werden kann. Zudem wiesen Kühl (1967) und Utz et al. (1993) bereits auf die gute Adaptationsfähigkeit der Patienten an neue Kondylenpositionen hin. Besonders die Auswirkung der Dysgnathiechirurgie auf die CMD kann noch nicht abschließend beurteilt werden und benötigt weitere Untersuchungen (Abrahamsson et al. 2007). Einige Studien verweisen in Bezug auf Muskelverhärtungen, funktionelle Beschwerden und Kopfschmerz auf eine symptomlindernde Auswirkung der Operation hin (Dervis und Tuncer 2002; Panula et al. 2000), dagegen konnten andere Autoren auch eine Verschlechterung feststellen (Onizawa et al. 1995). Eine direkte Beziehung zwischen gelenkbezogenen Beschwerden und der Dysgnathiechirurgie kann jedoch nicht gezogen werden.

Auch der Aspekt des Langzeitrezidives ist in Bezug auf die Nutzung von Gelenkpositionierungshilfen noch nicht ausreichend erforscht. Angelehnt an die Ergebnisse des Reviews von Costa et al. (2008) ist eine weitere Untersuchung der Notwendigkeit und Erfolgsquote von Methodiken zur intraoperativen Kondylenpositionierung empfehlenswert. Da nur ein geringer Anteil der Studien die diese Notwendigkeit untersucht haben, überhaupt Positionierungshilfen genutzt haben, steht eine abschließende Beurteilung noch aus.

Die hier gezeigte Studie erweitert die Untersuchung zur gelenkbezogenen Dysgnathieforschung um eine moderne Methodik und zeigt eine signifikante Auswirkung der Muskelaktivität auf die zentrische Kondylenposition auf Gelenkebene. Sie erweitert die bisherige sehr differente Studienlange und verweist deutlich auf die Notwenigkeit der präoperativ hergestellten Positionierungshilfen. Folgestudien in diesem Bereich der Dysgnathiechirurgie stehen noch aus und sind dringend empfehlenswert. 


\section{$5 \quad$ Zusammenfassung}

Diese einarmige, prospektive und monozentrische Studie setzte sich die Untersuchung der zentrischen Kondylenposition unter Einfluss der Allgemeinanästhesie im Rahmen der Dysgnathieoperation zum Ziel.

In die Untersuchung wurden die Kondylenpositionen von 30 Patienten an zwei verschiedenen Zeitpunkten (prä- und intraoperativ) einbezogen, die dreidimensionalen cephalometrisch und volumetrisch analysiert wurden. Alle Patienten unterzogen sich einer interdisziplinären Dysgnathietherapie in der Poliklinik für Kieferorthopädie und der Klinik für Mund-, Kiefer-, Gesichtschirurgie des Zentrums ZMK der Universitätsmedizin Göttingen. Das Patientengut setze sich aus 16 weiblichen und 14 männlichen Probanden zusammen, die zum Zeitpunkt der Operation durchschnittlich 25,8 Jahre alt waren. Unter Ihnen befanden sich 13 Patienten mit einer skelettalen Klasse II, 14 Patienten mit einer skelettalen Klasse III und drei Patienten mit einer skelettalen Klasse I.

Bei jedem Patienten wurden zwei Zentrikregistrate nach selbigem Schema durchgeführt, eines präoperativ im Rahmen der vorbereitenden Maßnahmen, eines intraoperativ unter Einfluss der Allgemeinanästhesie. Über die dreidimensionale Bildgebung und die Digitalisierung der okklusalen Verhältnisse und Rekonstruktion der Kondylenpositionen auf Basis dreidimensionaler Modelle erfolgte eine cephalometrische und volumetrische vergleichende Analyse der Positionen.

Die linearen Messungen ergaben keine statistisch signifikanten Änderungen der Gelenkposition. Erst die Analyse des kollidierenden Volumens ergab eine signifikante Stellungsänderung zwischen der prä- und intraoperativen Zentrik $(p=0,015)$. Eine Subgruppenanalyse zwischen Patienten mit skelettaler Klasse II und III, sowie asymmetrischen und symmetrischen Patienten ergaben keine signifikanten Ergebnisse.

Auf Basis der Ergebnisse dieser Studie scheint der strittige Einsatz von Hilfsmitteln zur Registrierung und Positionierung im Rahmen der Operationsplanung zur Prävention von Rezidiven und Vorhersagbarkeit der Therapie sinnvoll zu sein. Da die Diskrepanz zwischen den linearen und volumetrischen Ergebnissen recht groß ist, scheint eine intraorale Orientierung anhand der Okklusion fehlerbehaftet $\mathrm{zu}$ sein. Besondern bei variierenden Behandlern, sowohl auf kieferorthopädischer als auch kieferchirurgischer Seite, mit unterschiedlichen Erfahrungswerten in Bezug auf die zentrische Kondylenposition scheint in größeren Kliniken zum stabilen und vorhersagbaren Outcome der Operationsergebnisse eine präoperative Registrierung, wie sie an der UMG durchgeführt wird, unabdingbar. 


\section{$6 \quad$ Literaturverzeichnis}

Abrahamsson C, Ekberg EC, Henrikson T, Bondemark L (2007): Alterations of temporomandibular disorders before and after orthognathic surgery: a systematic review. Angle Orthod 77, 729-734

Al-Moraissi EA, Wolford LM, Perez D, Laskin DM, Ellis E (2017): Does Orthognathic Surgery Cause or Cure Temporomandibular Disorders? A Systematic Review and MetaAnalysis. J Oral Maxillofac Surg 75, 1835-1847

Andrews LF (1972): The six keys to normal occlusion. Am J Orthod 62, 296-309

American Association of Anesthesiologists (2014): ASA Physical Status Classification System https://www.asahq.org/standards-and-guidelines/asa-physical-status-classificationsystem, Zugriff am 05.05.2019

Angle E (1898): Double resection of the lower maxilla. Dent Cosmos $\underline{40}$

Aoyama S, Kino K, Kobayashi J, Yoshimasu H, Amagasa T (2005): Clinical evaluation of the temporomandibular joint following orthognathic surgery Multiple logistic regression analysis. J Med Dent Sci $\underline{52}, 109-114$

Bamber MA, Abang Z, Ng WF, Harris M, Linney A (1999): The effect of posture and anesthesia on the occlusal relationship in orthognathic surgery. J Oral Maxillofac Surg $\underline{57}$, 1164-1172; discussion 1172-1164

Baumrind S, Frantz RC (1971): The reliability of head film measurements: 1. Landmark identification. Am J Orthod $\underline{60}, 111-127$

Becker CM, Kaiser DA, Schwalm C, University of Colorado Health Sciences Center D

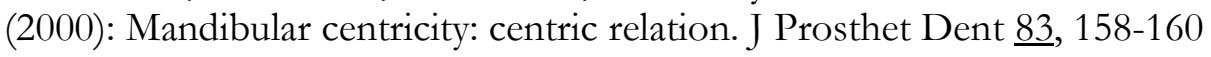

Bell WH, Epker BN (1976): Surgical-orthodontic expansion of the maxilla. Am J Orthod 70, 517-528

Bell WH, Jacobs JD (1981): Tridimensional planning for surgical/orthodontic treatment of mandibular excess. Am J Orthod $\underline{80}, 263-288$

Bettega G, Dessenne V, Raphaël B, Cinquin P (1996): Computer-assisted mandibular condyle positioning in orthognathic surgery. J Oral Maxillofac Surg $\underline{54}$, 553-558 
Bettega G, Cinquin P, Lebeau J, Raphael B (2002): Computer-assisted orthognathic surgery: clinical evaluation of a mandibular condyle repositioning system. J Oral Maxillofac Surg 60, 27-34; discussion 34-25

Bicaj T, Usami H, MericskeStern R, Kolgeci L, Katsoulis J, Bayer S, Enkling N (2013): Change of the condyle-position in maximum intercuspidation during digital, clinical occlusal analysis Veränderung der Kondylenposition in maximaler Interkuspidation bei der digitalen. J Craniomandib Funct $\underline{5}, 267-276$

Blair VP (1906): Report of a case of double resection for the correction of protrusion of the mandible. Dent Cosmos $\underline{48}, 817$

Blomqvist JE, Ahlborg G, Isaksson S, Svartz K (1997): A comparison of skeletal stability after mandibular advancement and use of two rigid internal fixation techniques. J Oral Maxillofac Surg 포, 568-574

Böhm A, Rammelsberg P, May H, Pho Duc J, Pospiech P, Gernet W (1995): Direkte dreidimensionale elektronische Kondylenpositionsanalysen zur Bestimmung von RKPIKP-Diskrepanzen. Dtsch Zahnärztl, 50

Borba AM, Ribeiro-Junior O, Brozoski MA, Ce PS, Espinosa MM, Deboni MC, Miloro M, Naclerio-Homem MG (2014): Accuracy of perioperative mandibular positions in orthognathic surgery. Int J Clin oral Maxillofac Surg $\underline{43}$, 972-979

Brooks RA (1977): A quantitative theory of the Hounsfield unit and its application to dual energy scanning. J Comput Assist Tomogr 1, 487-493

Brooks SL, Westesson P-L, Eriksson L, Hansson LG, Barsotti JB (1992): Prevalence of osseous changes in the temporomandibular joint of asymptomatic persons without internal derangement. Oral Surg Oral Med Oral Pathol $\underline{73}$, 118-122

Chen S, Lei J, Wang X, Fu KY, Farzad P, Yi B (2013): Short- and long-term changes of condylar position after bilateral sagittal split ramus osteotomy for mandibular advancement in combination with Le Fort I osteotomy evaluated by cone-beam computed tomography. J Oral Maxillofac Surg 71, 1956-1966

Cho SH, Schaefer O, Thompson GA, Guentsch A (2015): Comparison of accuracy and reproducibility of casts made by digital and conventional methods. J Prosthet Dent $\underline{113}$, 310-315

Christensen GJ (2004): Is occlusion becoming more confusing?: A plea for simplicity. J Am Dent Assoc 135, 767-770 
Claus J, Koerich L, Weissheimer A, Almeida M, de Oliveira RB (2019): Assessment of condylar changes after orthognathic surgery using computed tomography regional superimposition. Int J Clin oral Maxillofac Surg $\underline{48}, 1201-1208$

Cortese A, Chandran R, Borri A, Cataldo E (2019): A modified novel technique for condylar positioning in mandibular bilateral sagittal split osteotomy using computerassisted designed and computer-assisted manufactured surgical guides. J Oral Maxillofac Surg $\underline{77}$, 1069. e1061-1069. e1069

Costa AL, Barbosa BV, Perez-Gomes JP, Calle AJ, Santamaria MP, Lopes SC (2018): Influence of voxel size on the accuracy of linear measurements of the condyle in images of cone beam computed tomography: A pilot study. J Clin Exp Dent 10, e876-e882

Costa F, Robiony M, Toro C, Sembronio S, Polini F, Politi M (2008): Condylar positioning devices for orthognathic surgery: a literature review. Oral Surg Oral Med Oral Pathol Oral Radiol Endod 106, 179-190

Dal Pont G (1961): Retromolar osteotomy for the correction of prognathism. J Oral Surg Anesth Hosp Dent Serv 19, 42-47

Dawson PE (1979): Centric relation. Its effect on occluso-muscle harmony. Dent Clin North Am 23, 169-180

Dentistry JoP (2005): The glossary of prosthodontic terms. J Prosthet Dent $\underline{94}, 10-92$

Dervis E, Tuncer E (2002): Long-term evaluations of temporomandibular disorders in patients undergoing orthognathic surgery compared with a control group. Oral Surg Oral Med Oral Pathol Oral Radiol Endod 94, 554-560

Deutsche Gesellschaft für Funktionsdiagnostik und -therapie (2020): Befundbögen Klinischer Funktionsstatus https://www.dgfdt.de/richtlinien_formulare, Zugriff am 15.10.2020

DGZMK (2005): Terminologie der deutschen Gesellschaft für Funktionsdiagnistik und therapie und der deutschen Gesellschaft für zahnärztliche Prothetik und Werkstoffkunde https://www.dgzmk.de/terminologie-der-deutschen-gesellschaft-fuer-funktionsdiagnostikund-therapie-dgfdt-und-der-deutschen-gesellschaft-fuer-zahnaerztliche-prothetik-undwerkstoffkunde-dgzpw-, Zugriff am 06.04.2018 
Dowling PA, Espeland L, Krogstad O, Stenvik A, Kelly A (1999): Duration of orthodontic treatment involving orthognathic surgery. Int J Adult Orthodon Orthognath Surg $\underline{14}$, 146152

Eggensperger N, Smolka K, Luder J, Iizuka T (2006): Short- and long-term skeletal relapse after mandibular advancement surgery. Int J Clin oral Maxillofac Surg $\underline{35}$, 36-42

Ellis E (1994): Condylar positioning devices for orthognathic surgery: are they necessary? J Oral Maxillofac Surg 52, 536-552; discussion 552-534

Ellis E, Hinton RJ (1991): Histologic examination of the temporomandibular joint after mandibular advancement with and without rigid fixation: An experimental investigation in adult Macaca mulatta. J Oral Maxillofac Surg Surgery 노, 1316-1327

Epker BN, Wessberg GA (1982): Mechanisms of early skeletal relapse following surgical advancement of the mandible. Br J Oral Surg 20, 175-182

Eschler J (1963): Form und Funktion im Kausystem. Fortschr Kieferorthop 24, 247-265

Etz E, Hellmann D, Giannakopoulos N, Schmitter M, Rammelsberg P, Schindler H (2012): The variability of centric jaw relations in the process chain of prosthetic restorations and their neuromuscular effects Variabilität zentrischer Kieferrelationen in der Prozesskette prothetischer Restaurationen. J Craniomandib Funct 4, 141-156

Ferrario V, Sforza C, Jr Miani A Serrao G, Tartaglia G (1996): Open-close movements in the human temporomandibular joint: does a pure rotation around the intercondylar hinge axis exist? J Oral Rehabil 23 $\underline{2}, 401-408$

Gateno J, Xia JJ, Teichgraeber JF (2011): New 3-dimensional cephalometric analysis for orthognathic surgery. J Oral Maxillofac Surg $\underline{69}$, 606-622

Gateno J, Miloro M, H. Hendler B, Horrow M (1993): The use of ultrasound to determine the position of the mandibular condyle J Oral Maxillofac Surg $\underline{51}$, 1081-1086

Hanau RL (1929): Occlusal changes in centric relation. J Am Dent Assoc 16, 1903-1915

Häupl K (1960): Funktionelle Reizgeschehen und Umbau in den Kiefergelenksgeweben unter physiologischen und pathologischen Bedingungen. Fortschr Kiefer Gesichtschir $\underline{6}$, 28-39 
Hori M, Okaue M, Hasegawa M, Harada D, Kamogawa D, Matsumoto M, Tanaka H (1999): Worsening of pre-existing TMJ dysfunction following sagittal split osteotomy: a study of three cases. J Oral Sci $\underline{41}, 133-139$

Hullihen SP (1849): Case of Elongation of the under Jaw and Distortion of the Face and Neck, Caused by a Burn, Successfully Treated. Am J Dent Sci 2 , 157-165

Hunsuck EE (1968): A modified intraoral sagittal splitting technic for correction of mandibular prognathism. J Oral Surg 므, 250-253

Ireland AJ, Cunningham SJ, Petrie A, Cobourne MT, Acharya P, Sandy JR, Hunt NP (2014): An index of orthognathic functional treatment need (IOFTN). J Orthod 41, 77-83

Jacobson A (1975): The "Wits" appraisal of jaw disharmony. Am J Orthod 67, 125-138

Jacobson A (1976): Application of the "Wits" appraisal. Am J Orthod 70, 179-189

Jędrzejewski M, Smektała T, Sporniak-Tutak K, Olszewski R (2015): Preoperative, intraoperative, and postoperative complications in orthognathic surgery: a systematic review. Clin Oral Investig 19, 969-977

Jimenez I (1989): Electromyography of masticatory muscles in three jaw registration positions. Am J Orthod Dentofacial Orthop 모, 282-288

Kallela I, Laine P, Suuronen R, Iizuka T, Pirinen S, Lindqvist C (1998): Skeletal stability following mandibular advancement and rigid fixation with polylactide biodegradable screws. Int J Clin oral Maxillofac 27, 3-8

Kandasamy S, Boeddinghaus R, Kruger E (2013): Condylar position assessed by magnetic resonance imaging after various bite position registrations. Am J Orthod Dentofacial Orthop $\underline{144}, 512-517$

Keshvad A, Winstanley R (2001): An appraisal of the literature on centric relation. Part III. J Oral Rehabil 28, 55-63

Kim J-Y, Jung H-D, Jung Y-S, Hwang C-J, Park H-S (2014): A simple classification of facial asymmetry by TML system. J Craniomaxillofac Surg 42, 313-320

Kordaß B, Ruge S (2015): On the analysis of condylar path versus real motion of the temporomandibular joint: application for Sicat Function Analyse der Kondylenbahn im 
Vergleich zu den realen Bewegungen des Kiefergelenks-Möglichkeiten. Int J Comput Dent $\underline{18}, 225-235$

Kühl W (1967): Ergebnisse der sogenannten Handbißnahme in der Totalprothetik. Schweiz Monatsschr Zahnheilkd 7, 1120

Lauritzen AG: Atlas of occlusal analysis, 42. Johnson Publishing Co., Colorado 1974

Lehmann KM, Hellwig E: Einführung in die restaurative Zahnheilkunde: ein Lehrbuch für den propädeutischen Studienabschnitt. Urban \& Schwarzenberg, München 1998

Lentner E, Rammelsberg P, Böhm A, Pospiech P, Gernet W (1997): Zum Untersuchereinfluss auf Lage und Reproduzierbarkeit der zentrischen Kondylenposition. Dtsch Zahnärztl, 411-415

Leonard M (1976): Preventing rotation of the proximal fragment in the sagittal ramus split operation. J Oral Surg $\underline{34}, 942$

Lindqvist C, Söderholm A-L (1988): A Simple Method for Establishing the Position of the Condylar Segment in Sagittal Split Osteotomy of the Mandible. Plast Reconstr Surg $\underline{82}$

Luhr H-G (1989): The Significance of Condylar Position Using Rigid Fixation in Orthognathic Surgery. Clin Plast Surg 16, 147-156

Luhr H-G, Kubein-Meesenburg D, Schwestka-Polly R (1991): Bedeutung und Technik der Kiefergelenkpositionierung bei der sagittalen Spaltung des Unterkiefers. Fortschr Kieferorthop $\underline{52}$, 66-72

Luhr H-G, Schauer W, Jäger A, Kubein-Meesenburg D (1986): Formveränderung des Unterkiefers durch kieferorthopädisch-chirurgische Maßnahmen mit stabiler Fixation der Segmente. Fortschr Kieferorthop 47, 39-47

Maeda M, Itou S, Ishii Y, Yamamoto K, Kawamura Y, Matsuda T, Hayashi N, Ishii Y (1992): Temporomandibular joint movement: evaluation of protrusive splint therapy with GRASS MR imaging. Acta radiol $\underline{33}, 410-413$

McCollum B (1960): The mandibular hinge axis and a method of locating it. J Prosthet Dent $\underline{10}, 428-435$ 
Michel C: Zur Problematik der Gelenkposition in der orthopädischen Chirurgie des KieferGesichtsschädels. Habilitationsschirft, Universität Würzburg 1990

Moss JP, Chalmers CP (1974): An electromyographic investigation of patients with a normal jaw relationship and a Class III jaw relationship. Am J Orthod $\underline{66}, 538-556$

Nova I, Kallus S, Berger M, Ristow O, Eisenmann U, Freudlsperger C, Hoffmann J, Dickhaus H (2017): Computer assisted positioning of the proximal segment after sagittal split osteotomy of the mandible: Preclinical investigation of a novel electromagnetic navigation system. J Oral Maxillofac Surg 45, 748-754

Obwegeser H (1964): The indications for surgical correction of mandibular deformity by the sagittal splitting technique. Br J Oral Surg 1, 157-171

Olmez H, Gorgulu S, Akin E, Bengi AO, Tekdemir İ, Ors F (2011): Measurement accuracy of a computer-assisted three-dimensional analysis and a conventional two-dimensional method. Angle Orthod $\underline{81}, 375-382$

Onizawa K, Schmelzeisen R, Vogt S (1995): Alteration of temporomandibular joint symptoms after orthognathic surgery: comparison with healthy volunteers. J Oral Maxillofac Surg 프, 117-121

Paknahad M, Shahidi S (2015): Association between mandibular condylar position and clinical dysfunction index. J Craniomaxillofac Surg 43, 432-436

Palaskar JN, Murali R, Bansal S (2013): Centric relation definition: a historical and contemporary prosthodontic perspective. J Indian Prosthodont Soc $\underline{13}$, 149-154

Panula K, Somppi M, Finne K, Oikarinen K (2000): Effects of orthognathic surgery on temporomandibular joint dysfunction: A controlled prospective 4-year follow-up study. Int J Clin Oral Maxillofac Surg 29, 183-187

Paulus G (1983): Rezidivverhalten nach Ramusosteotomien mit unterschiedlichen Osteosyntheseformen. Dtsch. Zahnärztl. Z. $\underline{38}$, 51-55

Pittayapat P, Limchaichana-Bolstad N, Willems G, Jacobs R (2014): Three-dimensional cephalometric analysis in orthodontics: a systematic review. Orthod Craniofac Res $\underline{17}, 69$ 91 
Politi M, Toro C, Costa F, Polini F, Robiony M (2007): Intraoperative Awakening of the Patient During Orthognathic Surgery: A Method to Prevent the Condylar Sag. J Oral Maxillofac Surg 65, 109-114

Proffit WR, Phillips C, Dann Ct (1990): Who seeks surgical-orthodontic treatment? Int J Adult Orthodon Orthognath Surg $\underline{5}, 153-160$

Proffit WR, Phillips C, Tulloch JF, Medland PH (1992): Surgical versus orthodontic correction of skeletal Class II malocclusion in adolescents: effects and indications. Int J Adult Orthodon Orthognath Surg 7, 209-220

Proffit WR, Sarver DM: Combined Surgical and Orthodontic Treatment in: Proffit WR, Fields HW, Larson B, Sarver DM (Hrsg.): Contemporary orthodontics. 6. Auflage; Elsevier Health Sciences, Philadelphia 2019, 657-709

Puelacher W, Waldhart E (1987): [Follow-up after surgical correction of mandibular prognathism]. Z Stomatol 84, 383-389

Pullinger AG, Solberg WK, Hollender L, Guichet D (1986): Tomographic analysis of mandibular condyle position in diagnostic subgroups of temporomandibular disorders. J Prosthet Dent $\underline{55}, 723-729$

Reyneke JP, Ferretti C (2002): Intraoperative diagnosis of condylar sag after bilateral sagittal split ramus osteotomy. Br J Oral Maxillofac Surg 40, 285-292

Rinchuse DJ, Kandasamy S (2006): Centric relation: a historical and contemporary orthodontic perspective. J Am Dent Assoc 137, 494-501

Rotskoff KS, Herbosa EG, Villa P (1991): Maintenance of condyle-proximal segment position in orthognathic surgery. J Oral Maxillofac Surg 49, 2-7

Saka B, Petsch I, Hingst V, Härtel J (2004): The influence of pre- and intraoperative positioning of the condyle in the centre of the articular fossa on the position of the disc in orthognathic surgery. A magnetic resonance study. Br J Oral Maxillofac Surg 42, 120-126

Scapino RP (1997): Morphology and mechanism of the jaw joint. Science and practice of occlusion, 23-40

Schindler H, Lenz J, Türp J, Schweizerhof K, Rues S (2009): Small unilateral jaw gap variations: equilibrium changes, co-contractions and joint forces. J Oral Rehabil $\underline{36}, 710$ 718 
Schupp W, Ehmer U, Wegener H (1992): Vergleich der klinischen und instrumentellen Funktionsbefunde bei Probanden mit einer Angle-Klasse II/2 gegenüber einer Eugnathengruppe. Fortschr Kieferorthop $\underline{53}$, 84-87

Schwenzer N, Ehrenfeld M (1990): Chirurgische Kieferorthopädie. Schwenzer und Grimm (Hrsg.) Spezielle Chirurgie 2, 463-518

Schwestka R, Engelke D, Kubein-Meesenburg D (1990): Condylar position control during maxillary surgery: the condylar positioning appliance and three-dimensional double splint method. Int J Adult Orthodon Orthognath Surg $\underline{5}, 161-165$

Schwestka-Polly R (2004): Fortschritte in der gelenkbezüglichen kieferorthopädischkieferchirurgischen Therapie. Inf Orthod Kieferorthop $\underline{36}$, 205-218

Segner D, Hasund A: Klinische Relevanz der Analyse. In Dietmar Segner (Hrsg.): Individualisierte kephalometrie. 3. Auflage; Segner Verlag, Hamburg 1998, 112-123

Shokri A, Zarch HH, Hafezmaleki F, Khamechi R, Amini P, Ramezani L (2019): Comparative assessment of condylar position in patients with temporomandibular disorder (TMD) and asymptomatic patients using cone-beam computed tomography. Dent Mes Probl 트, 81-87

Simpson W (1974): The results of surgery for mandibular prognathism. Br J Oral Surg 12 , 166-176

Smith Jr HF (1975): A comparison of empirical centric relation records with location of terminal hinge axis and apex of the gothic arch tracing. J Prosthet Dent $\underline{33}, 511-520$

Solberg WK, Bibb CA, Nordström BB, Hansson TL (1986): Malocclusion associated with temporomandibular joint changes in young adults at autopsy. Am J Orthod $\underline{89}$, 326-330

Steinhäuser EW (1996): Historical development of orthognathic surgery. J Craniomaxillofac Surg 24, 195-204

Thilander B, Rubio G, Pena L, de Mayorga C (2002): Prevalence of temporomandibular dysfunction and its association with malocclusion in children and adolescents: an epidemiologic study related to specified stages of dental development. Angle Orthod $\underline{72}$, $146-154$ 
Thompson JR (1946): The rest position of the mandible and its significance to dental science. J Am Dent Assoc 33, 151-180

Toll DE, Popović N, Drinkuth N (2010): The Use of MRI Diagnostics in Orthognathic Surgery. Fortschr Kieferorthop 1ㅡ, 68-80

Tripodakis A, Smulow J, Mehta N, Clark R (1995): Clinical study of location and reproducibility of three mandibular positions in relation to body posture and muscle function. J Prosthet Dent $\underline{73}$, 190-198

Troy BA, Shanker S, Fields HW, Vig K, Johnston W (2009): Comparison of incisor inclination in patients with Class III malocclusion treated with orthognathic surgery or orthodontic camouflage. Am J Orthod Dentofacial Orthop 135, 146.e141-146.e149

Ueki K, Marukawa K, Shimada M, Yoshida K, Hashiba Y, Shimizu C, Nakgawa K, Alam S, Yamamoto E (2007): Condylar and disc positions after intraoral vertical ramus osteotomy with and without a Le Fort I osteotomy. Int Clin Oral Maxillofac Surg 즈, 207-213

Utz KH, Bernard N, Hültenschmidt R, Wegmann U, Kurbel R (1993): [The reproducibility of the manual checkbite in the wearers of complete dentures]. Schweiz Monatsschr Zahnmed 103, 561-566

Utz KH, Müller F, Lückerath W, Fuss E, Koeck B (2002): Accuracy of check-bite registration and centric condylar position. J Oral Rehabil 29, 458-466

Van Sickels JE, Dolce C, Keeling S, Tiner BD, Clark GM, Rugh JD (2000): Technical factors accounting for stability of a bilateral sagittal split osteotomy advancementWire osteosynthesis versus rigid fixation. Oral Surg Oral Med Oral Pathol Oral Radiol Endod $\underline{89}, 19-23$

Wallen T, Bloomquist D (1986): The clinical examination: is it more important than cephalometric analysis in surgical orthodontics? Int J Adult Orthodon Orthognath Surg 1 , 179-191

Wang M-Q, He J-J, Wang K, Svensson P (2009): Influence of changing occlusal support on jaw-closing muscle electromyographic activity in healthy men and women. Acta Odontol Scand $\underline{67}, 187-192$

Weffort SYK, de Fantini SM (2010): Condylar displacement between centric relation and maximum intercuspation in symptomatic and asymptomatic individuals. Angle Orthod $\underline{80}$, $835-842$ 
Weinberg LA (1983): The role of stress, occlusion, and condyle position in TMJ dysfunction-pain. J Prosthet Dent $\underline{49}$, 532-545

Whipple JW (1898): Double resection of the inferior maxilla for protruding lower jaw. Dent Cosmos 40, 552-557

Whyms BJ, Vorperian HK, Gentry LR, Schimek EM, Bersu ET, Chung MK (2013): The effect of computed tomographic scanner parameters and 3-dimensional volume rendering techniques on the accuracy of linear, angular, and volumetric measurements of the mandible. Oral Surg Oral Med Oral Pathol Oral Radiol 115, 682-691

Wolford LM, Reiche-Fischel O, Mehra P (2003): Changes in temporomandibular joint dysfunction after orthognathic surgery. J Oral Maxillofac Surg 61, 655-660

Worms FW, Speidel TM, Bevis RR, Waite DE (1980): Posttreatment stability and esthetics of orthognathic surgery. Angle Orthod $\underline{50}$, 251-273

Yaedú RYF, Mello MdAB, Tucunduva RA, da Silveira JSZ, Takahashi MPMS, Valente ACB (2017): Postoperative orthognathic surgery edema assessment with and without manual lymphatic drainage. J Craniofac Surg $\underline{28}$, 1816-1820

Yaghmaei M, Ejlali M, Nikzad S, Sayyedi A, Shafaeifard S, Pourdanesh F (2013): General anesthesia in orthognathic surgeries: does it affect horizontal jaw relations? J Oral Maxillofac Surg 1ㅜ, 1752-1756

Yamada K, Hanada K, Hayashi T, Ito J (2001): Condylar bony change, disk displacement, and signs and symptoms of TMJ disorders in orthognathic surgery patients. Oral Surg Oral Med Oral Pathol Oral Radiol Endod 91, 603-610

Yang HJ, Kim DS, Yi W-J, Hwang SJ (2013): Reduced joint distance during TMJ movement in the posterior condylar position. J Oral Maxillofac Surg 41, e159-e164

Yoon SY, Song JM, Kim YD, Chung IK, Shin SH (2015): Clinical changes of TMD and condyle stability after two jaw surgery with and without preceding TMD treatments in class III patients. Maxillofac Plast Reconstr Surg $\underline{37}, 9$

Zafar H, Choi DS, Jang I, Cha BK, Park YW (2014): Positional change of the condyle after orthodontic-orthognathic surgical treatment: is there a relationship to skeletal relapse? J Korean Assoc Oral Maxillofac Surg 누, 160-168 
Zak MJ, Dolan EA, Angelillo JC, McGraw TA (1992): No effect of neuromuscular blockade on the temporomandibular joint position during general anesthesia. Anesth Prog $\underline{39}, 212-214$

Zamora N, Llamas JM, Cibrián R, Gandia JL, Paredes V (2012): A study on the reproducibility of cephalometric landmarks when undertaking a three-dimensional (3D) cephalometric analysis. Med Oral Patol Oral Cir Bucal bucal 17, e678 


\section{Danksagung}

An dieser Stelle möchte ich meinen besonderen Dank Prof. Dr. med. dent. Philipp MeyerMarcotty, Direktor der Poliklinik für Kieferorthopädie der Universitätsmedizin Göttingen, aussprechen, für die Überlassung dieses Forschungsthemas und die hervorragende Betreuung.

Prof. Dr. med. Dr. med. dent. Henning Schliephake, Direktor der Klinik für Mund-, Kiefer-, Gesichtschirurgie der Universitätsmedizin Göttingen, danke ich für die konstruktive Kritik und die wissenschaftliche Zweitbetreuung.

Besonderer Dank gilt Dr. med. dent. Anja Quast für die exzellente Betreuung, die nahezu unerschöpfliche Diskussions- und Hilfsbereitschaft und den wertvollen fachlichen Input, ohne die diese Arbeit nie zustande gekommen wäre.

Weiterer Dank gilt der Arbeitsgruppe Dysgnathie für die gute Arbeitsatmosphäre und die hilfreiche Zusammenarbeit, dem Team der Poliklinik für Kieferorthopädie, sowie dem Operationsteam der Mund-, Kiefer-, Gesichtschirurgie der Universität Göttingen für die komplikationslose Ermöglichung der Untersuchungsdurchführung. 